\title{
AUTOMATISIERTER UND VERNETZTER VERKEEHR IM LANGEN LEVEL 4
}

SIEDLUNGSENTWICKLUNG, VERKEHRSPOLITIK UND PLANUNG WÄHREND DER ÜBERGANGSZEIT 
4.1

\section{TECHNOLOGISCHE ENTWICKLUNGEN AUTOMATISIERTER UND VERNETZTER FAHRZEUGE: WO STEHEN WIR HEUTE?}

Abbildung 4.1.1: Ein LIDAR-Sensor am Dach des Testautos „Homer“

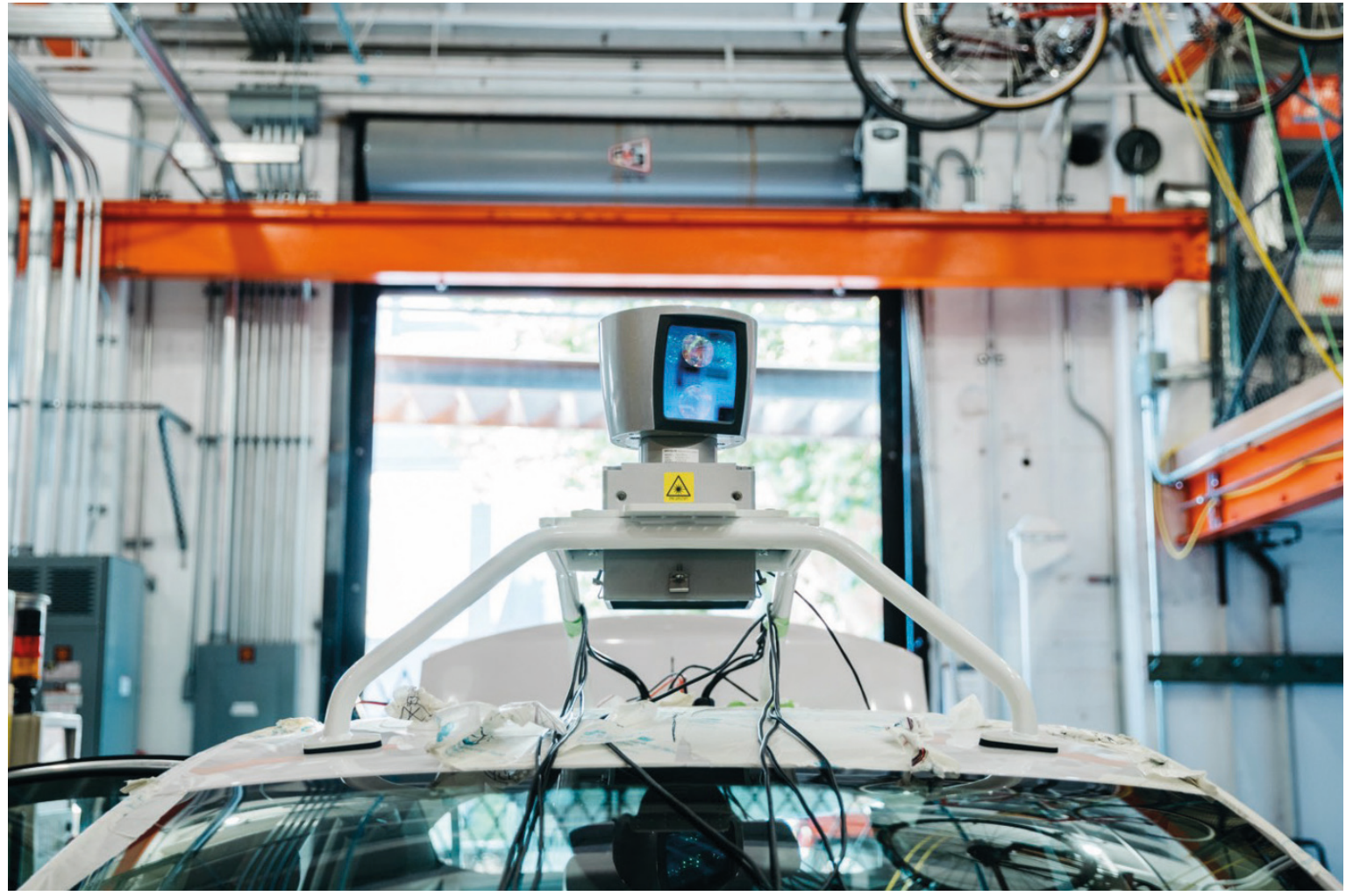

Quelle: voyage.auto

Automatisierung und Vernetzung sind die entscheidenden Treiber des Wandels des Verkehrssystems, allerdings zwei grundsätzlich verschiedene Trends, die nicht zwingend miteinander verbunden sind (Perret et al. 2017, S. 6). Ihre faktische Gleichzeitigkeit und parallele Entwicklung wird allerdings immer stärker betont: Während frühere Forschungsarbeiten noch häufig vom autonomen Fahren oder autonomen Fahrzeugen gesprochen haben, findet sich in neueren Beiträgen verstärkt der Begriff ,automatisiertes und vernetztes Fahrzeug“: „Even though automated vehicles do not necessarily need to be connected and connected vehicles do not require automation, it is expected that in the medium term connectivity will be a major enabler for automated vehicles“ (Europäische Kommission 2018, S. 4).

Als Reaktion auf die vorherrschende begriffliche Vielfalt und deren unklarer bzw. irreführender Bedeutungen rät SAE International (2018, S. 28) von den Begriffen „selbstfahrendes Fahrzeug“, ,autonomes Fahrzeug“,
„Fahrroboter“ explizit ab. Was gemeinhin als ,selbstfahrendes“ oder ,autonomes“ Fahrzeug benannt wird, entspricht in der vorgeschlagenen und hier durchgängig verwendeten Terminologie dem ,voll automatisierten Fahrzeug.“

Die Gleichzeitigkeit von Automatisierung und Vernetzung von Fahrzeugen liegt darin begründet, dass schon heute eine immer stärkere Vernetzung für einige Fahraufgaben als Voraussetzung gesehen wird. So könnten Informationen über die aktuelle Verkehrssituation, den Zustand der Fahrbahn und möglicherweise Informationen von der Infrastruktur selbst (Ampeln, Mautstellen etc.) für das Fahrzeug notwendig sein, um einen sicheren automatisierten Betrieb zu ermöglichen (Ritz 2018, S. 184). Außerdem werden sich einige der gewünschten Auswirkungen von automatisierten Fahrzeugen nur einstellen, wenn diese auch vernetzt agieren oder kooperieren. Die Steigerung der Effizienz auf Straßen und im Straßennetz durch Verteilung, Erhöhung des Fahr- 
zeugdurchsatzes oder Sicherheit sind Beispiele dafür (Kagermann 2017, S. 363; Shladover 2018, S. 196). Diese Sicht wird von der Europäischen Kommission über diverse Initiativen und Förderprogramme (CAM, C-ITS, C-Roads) vertreten (Europäische Kommission 2018, S. 4).

Im Weiteren bedeutet das: Obwohl die meisten der ersten verfügbaren automatisierten Fahrsysteme, die automatisierte Fahrfunktionen der unteren Stufen bieten, noch relativ eigenständig und nicht bzw. kaum vernetzt sind, wird es auf längere Sicht - wenn höhere Stufen von Automatisierung erreicht werden - von Wichtigkeit sein, dass automatisierte Fahrsysteme weitestgehend vernetzt sind, so dass die gewünschten Effekte auch eintreten (Abb. 4.1.2; Shladover 2018, S. 193).
Abbildung 4.1.2: Schematische Darstellung der Unterscheidung zwischen eigenständigen, kooperativen und automatisierten Systemen

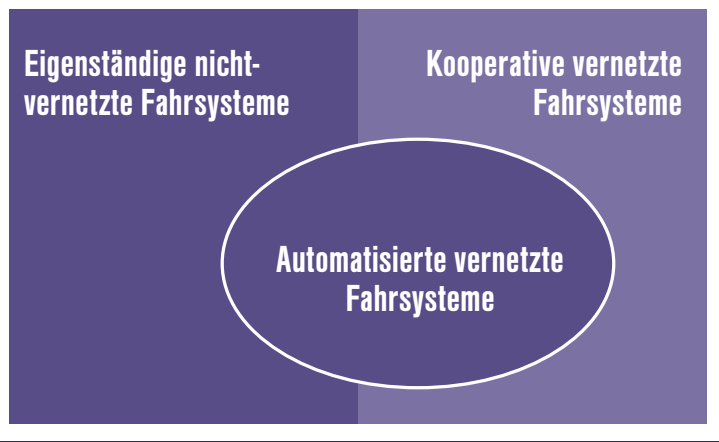

Quelle: AVENUE21 nach Shladover (2018)

Abbildung 4.1.3: Stufen der Automatisierung von Fahrsystemen

\begin{tabular}{|c|c|c|c|c|c|c|}
\hline LEVEL & NAME & BESCHREIBUNG & $\begin{array}{c}\text { QUER- UND } \\
\text { LÄNGSFÜHRUNG }\end{array}$ & $\begin{array}{l}\text { UMGEBUNGS- } \\
\text { BEOBACHTUNG }\end{array}$ & $\begin{array}{l}\text { RÜCKFALL- } \\
\text { EBENE }\end{array}$ & $\begin{array}{l}\text { OPERATIONAL } \\
\text { DESIGN DOMAIN }\end{array}$ \\
\hline \multicolumn{7}{|c|}{ FAHRER/IN FÜHRT ALLE FAHRAUFGABEN AUS } \\
\hline 0 & $\begin{array}{c}\text { KEINE } \\
\text { AUTOMATION }\end{array}$ & $\begin{array}{l}\text { FahrerIn fährt eigenständig, } \\
\text { auch wenn unterstützende } \\
\text { Systeme vorhanden sind. }\end{array}$ & FAHRER/IN & FAHRER/IN & KEINE & $N / A$ \\
\hline 1 & $\begin{array}{l}\text { ASSISTENZ- } \\
\text { SYSTEME }\end{array}$ & $\begin{array}{l}\text { Fahrassistenzsysteme helfen } \\
\text { bei der Fahrzeugbedienung } \\
\text { bei Längs- oder Querführung } \\
\text { (nicht gleichzeitig). }\end{array}$ & $\begin{array}{l}\text { FAHRER/IN UND } \\
\text { SYSTEM }\end{array}$ & FAHRER/IN & FAHRER/IN & LIMITIERT \\
\hline 2 & $\begin{array}{l}\text { TEILAUTO- } \\
\text { MATISIERUNG }\end{array}$ & $\begin{array}{l}\text { Ein oder mehrere } \\
\text { Fahrassistenzsysteme helfen } \\
\text { bei der Fahrzeugbedienung } \\
\text { bei Längs- und gleichzeitiger } \\
\text { Querführung, FahrerIn muss } \\
\text { System dauerhaft überwachen. }\end{array}$ & SYSTEM & FAHRER/IN & FAHRER/IN & LIMITIERT \\
\hline \multicolumn{7}{|c|}{ SYSTEM FÜHRT ALLE FAHRAUFGABEN AUS } \\
\hline 3 & $\begin{array}{l}\text { BEDINGTE } \\
\text { AUTOMA- } \\
\text { TISIERUNG }\end{array}$ & $\begin{array}{l}\text { Automatisiertes Fahren mit } \\
\text { der Erwartung, dass FahrerIn } \\
\text { auf Anforderung zum } \\
\text { Eingreifen reagieren muss. }\end{array}$ & SYSTEM & SYSTEM & $\begin{array}{l}\text { RÜCKFALL- } \\
\text { BEREITE/R } \\
\text { NUTZER/IN }\end{array}$ & LIMITIERT \\
\hline 4 & $\begin{array}{l}\text { HOCHAUTO- } \\
\text { MATISIERUNG }\end{array}$ & $\begin{array}{l}\text { Automatisierte Führung } \\
\text { des Fahrzeugs mit der } \\
\text { Erwartung, dass FahrerIn auf } \\
\text { Anforderung zum Eingreifen } \\
\text { reagiert. Ohne menschliche } \\
\text { Reaktion steuert das Fahrzeug } \\
\text { weiterhin autonom. FahrerIn } \\
\text { muss System nicht dauerhaft } \\
\text { überwachen. }\end{array}$ & SYSTEM & SYSTEM & SYSTEM & LIMITIERT \\
\hline 5 & $\begin{array}{l}\text { VOLLAUTOMA- } \\
\text { TISIERUNG }\end{array}$ & $\begin{array}{l}\text { Vollständig automatisiertes } \\
\text { Fahren, bei dem die } \\
\text { dynamische Fahraufgabe } \\
\text { unter jeder Fahrbahn- und } \\
\text { Umgebungsbedingung wie } \\
\text { von einem/r menschlichen } \\
\text { FahrerIn durchgeführt wird. }\end{array}$ & SYSTEM & SYSTEM & SYSTEM & UNLIMITIERT \\
\hline
\end{tabular}




\section{AUTOMATISIERUNGSLEVEL UND IHRE BEDEUTUNG}

Als automatisiert werden grundsätzlich Fahrzeuge unterschiedlicher Größen verstanden, die einen großen Anteil der Fahrleistungen eigenständig übernehmen bis hin zu fahrerlos betrieben werden können (Abb. 4.1.3). Für Fahrzeuge im Personen- und Güterverkehr gibt es unterschiedliche Einteilungen in Automatisierungsstufen, wobei sich in der wissenschaftlichen Debatte die Einteilung J3016 der SAE (Society of Automotive Engineers) international durchgesetzt hat.

Jene Systeme, die der/die FahrerIn nicht mehr dauerhaft überwachen muss, da die Längs- und Querführung in spezifischen Anwendungsfällen (Straßentypen, Geschwindigkeitsbereiche und Umfeldbedingungen) automatisiert ausgeführt wird, werden als bedingt automatisierte Fahrzeuge (,conditional driving automation“) bezeichnet. Der/die FahrerIn muss jedoch potenziell in der Lage sein, zu übernehmen, wenn das System die Übernahme fordert (Level 3). Als hochautomatisierte Fahrzeuge, die der Gegenstand vorliegender Studie sind, werden jene bezeichnet, deren Systeme alle Situationen automatisch bewältigen können, doch lediglich in einem dafür ausgelegten Bereich (ODD-spezifisch) fahrerlos betrieben werden bzw. die Fahraufgabe übernehmen. Es findet keine Übernahmeaufforderung statt (Level 4). Fahrzeuge deren Systeme die Fahraufgabe vollumfänglich für alle Straßentypen, Geschwindigkeitsbereiche und Umfeldbedingungen übernehmen (unabhängig von der ODD) und die somit von Start bis Ziel ohne FahrerIn betrieben werden, bezeichnet man schließlich als vollautomatisierte Fahrzeuge (Level 5; SAE International 2018, S. 19).

Die Operational Design Domain beschreibt dabei die Umfeldbedingungen, innerhalb derer ein automatisiertes Fahrsystem funktioniert. Parameter dieses Umfelds können unter anderem sein: geographische Lage, Straßentyp, Umwelt, Verkehr, Geschwindigkeit, Zeit (s. Abb. 4.1.4; SAE International 2018, S. 12).

Abbildung 4.1.4: Operational Design Domain

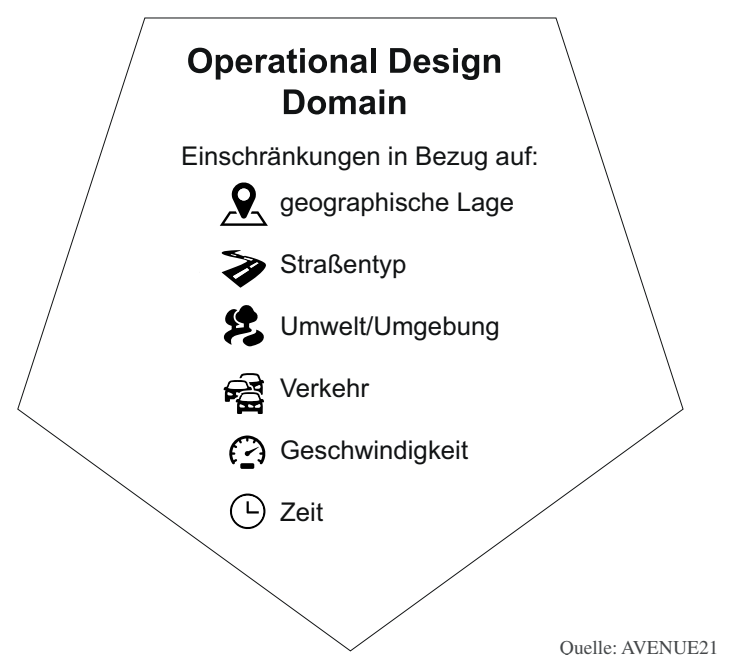

DAS LEVEL 4: AUTOMATISIERTE FAHRSYSTEME FÜR SPEZIFISCHE UMFELDBEDINGUNGEN (OPERATIONAL DESIGN DOMAINS)

Die Divergenz zwischen automatisierten Fahrsystemen (,Automated Driving Systems“ - ADS; SAE International 2018), die in eingeschränkten Umgebungen (ODD-spezifisch) funktionieren (Level 4), und jenen, die uneingeschränkt (ODD-unspezifisch) funktionieren (Level 5), ist tatsächlich enorm.

Außerhalb oder bei zu starker Veränderung dieser spezifischen Umfeldbedingungen bzw. der ODD ist die Funktionstüchtigkeit der automatisierten Fahrsysteme des Level 4 hingegen nicht mehr gegeben (NHTSA 2017, S. 6). Ist dies - also ein Übergang in das manuelle Fahren - notwendig wird der/die FahrerIn aufgefordert, die Fahrfunktion zu übernehmen. Ist diese/r dazu nicht imstande, wird das Fahrzeug in einen risikominimierten Systemzustand zurückgeführt (VDA 2015, S. 15; Wagner \& Kabel 2018, S. 317).

Automatisierte Fahrsysteme bzw. Anwendungen des Level 4 sind seit den 1990er Jahren im Einsatz. Ein bekanntes Beispiel in Europa ist das Parkshuttle, das in Rotterdam als Zubringerlinie auf der letzten Meile den Rivium Business Park mit der Metro Rotterdam verbindet. In der zweiten Generation operiert das Parkshuttle seit 2006 auf einer Strecke von rund fünf Kilometern mit ebenso vielen Stationen. Die Limitierungen der ODD wurden hier infrastrukturell gelöst: Das Parkshuttle fährt auf einer asphaltierten Trasse, die an beiden Seiten mit einem ein Meter hohen Zaun und einer Hecke von der Umgebung abgegrenzt ist.

Der Fortschritt der Technologie könnte jedoch zukünftig ein Operieren automatisierter Fahrsysteme des Level 4 ohne solch große Infrastrukturmaßnahmen wie eine bauliche Trennung bzw. eigene Fahrstreifen etc. ermöglichen (Hollestelle 2018, S. 24). Letztlich dienen solche Maßnahmen jedoch immer dazu, die Komplexität der ODD des automatisierten Fahrsystems (z. B. Vermeidung der Interaktion mit RadfahrerInnen) und damit auch die Anforderungen an das automatisierte Fahrsystem zu reduzieren.

\section{AUSGEWÄHLTE AUTOMATISIERTE FAHRSYSTEME DES LEVEL 4, IHRE OPERATIONAL DESIGN DOMAIN UND EIN- SATZFELDER}

Automatisierte Fahrsysteme des Level 4 können unterschiedliche Einsatzszenarien, sogenannte Use Cases aufweisen (Wachenfeld et al. 2015, S. 12), die letztlich durch die Eigenschaften ihrer jeweiligen ODD sowie weitere Merkmale, beispielsweise das mögliche Einsatzfeld bzw. Nutzungskonzept, gekennzeichnet sind. 


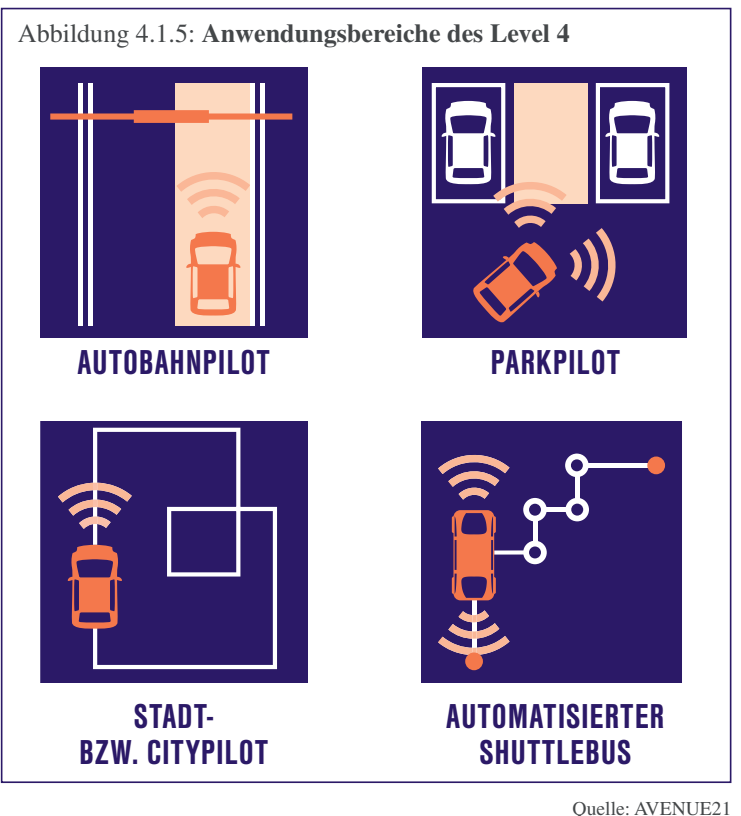

Verschiedene derzeit diskutierte automatisierte Fahrsysteme des Level 4 können dabei sehr unterschiedliche ODD sowie auch verschiedene mögliche Einsatzfelder aufweisen (Shladover 2018, S. 194). Dadurch ergeben sich zahlreiche mögliche Einsatzszenarien bzw. Use Cases solcher automatisierter Fahrsysteme (Abb. 4.1.5).

Im Folgenden werden ausgewählte, derzeit diskutierte automatisierte Fahrsysteme des Level 4 sowie ihre ODD und Einsatzfelder beschrieben. Die Auswahl beschränkt sich dabei auf vier Anwendungen.

\section{AUTOBAHNPILOT}

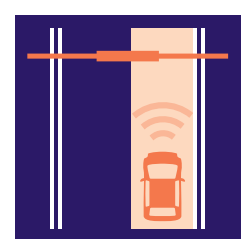

Beim Autobahnpiloten übernimmt das System ausschließlich auf Autobahnen oder autobahnähnlichen Schnellstraßen die Fahraufgabe - während der Fahrt auf der Autobahn ist kein Situationsbewusstsein von den InsassInnen gefordert, sie können anderen Tätigkeiten nachgehen (Wachenfeld et al. 2015, S. 12). Die Entwicklung von Autobahnpiloten wird vor allem von Fahrzeugherstellern vorangetrieben, befindet sich derzeit jedoch noch in der Entwicklung: Mit dem neuen A8 plant Audi beispielsweise gerade, das erste Serienfahrzeug mit einem Autobahnchauffeur (Level 3) auf den Markt zu bringen, der jedoch nur einfache Fahraufgaben bei gutem Wetter beherrscht. Wie eingangs beschrieben, muss der Fahrer daher noch potenziell in der Lage sein, zu übernehmen, wenn das System zur Übernahme auffordert (Schrepfer et al. 2018, S. 32; Ritz 2018, S. 30). Die weitere Entwicklung der Fahrzeughersteller wird jedoch in Richtung einer stetigen Steigerung der mögli- chen zu bewältigenden Fahraufgaben auf der Autobahn hin zu einem Autobahnpiloten des Level 4 gehen, der es dem/der FahrerIn erlaubt, ein Buch zu lesen oder zu schlafen, solange der Autobahnpilot auf der Autobahn fährt (Ritz 2018, S. 31). Dies könnte nicht nur den/die FahrerIn eines Pkws, sondern auch eines Nutzfahrzeugs bzw. Lkws entlasten (Eckstein et al. 2018, S. 9) sowie zudem bei einem Fernbus Anwendung finden.

Insbesondere hinsichtlich Lkws werden häufig Autobahnpiloten auf allein für Lkw gestatteten Fahrstreifen diskutiert, um die Anforderungen an das Fahrsystem zu reduzieren. Diese Anpassungen der ODD erfolgen vor dem Hintergrund, ein solches Fahrsystem des Level 4 letztlich schneller auf die Straße zu bringen (Shladover 2018, S. 194).

\section{PARKPILOT (AUTOMATISIERTES VALET-PARKEN)}

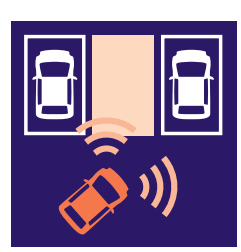

Offensichtlich scheint sich die Automatisierung des Privatwagens mit Blick auf die Fahrzeughersteller vor allem auf Autobahnen oder autobahnähnliche Straßen zu fokussieren (Schrepfer et al. 2018, S. 34). Aktuell wird jedoch von Herstellern wie beispielsweise Audi oder Daimler gleichfalls an der Entwicklung von Parkpiloten, also automatisierten Fahrsystemen, die ein automatisiertes Valet-Parken ermöglichen, gearbeitet (Ritz 2018, S. 30).

Beim automatisierten Valet-Parken fährt das automatisierte Fahrsystem, nachdem die PassagierInnen es verlassen haben, in eine nahe oder auch entfernte Parkposition. Der/die FahrerIn spart sich so die Zeit für die Parkplatzsuche oder das Abstellen des Fahrzeugs (Wachenfeld et al. 2015, S.15; Shladover 2018, S. 194). Derzeit wird ein solches automatisiertes Valet-Parken vor allem in Parkhäusern (oder Parkplätzen), also nicht im Straßennetz, sondern in als Sonderareale beschreibbaren Gebieten getestet: Daimler und Bosch beispielsweise testen in einem Parkhaus in Stuttgart ein solches System, bei dem das Fahrzeug einfach in einer ,Dropoff Area“ abgestellt wird, nach einem Befehl mittels Smartphone dann automatisiert parkt und nach einem abermaligem Befehl mittels Smartphone wieder automatisiert in eine „Pick-up Area“ zum Abholen fährt (Daimler 2018).

In Zukunft könnten solche automatisierten Fahrsysteme jedoch nicht nur in Sonderarealen, sondern auch in definierten freigegebenen Bereichen des niederrangigen Straßennetzes (z. B. Bereiche in der Innenstadt) operieren. Der/die FahrerIn kann so beispielsweise direkt vor einem Restaurant anhalten und nach dem 
Aussteigen dem Fahrzeug den Auftrag geben, automatisiert einen Parkplatz zu suchen, anzusteuern und dort zu parken (Eckstein et al. 2018, S. 9). Der Parkpilot würde aber dann den Bedingungen des Stadtpilots (s. nächster Absatz) sehr nahekommen. Aufgrund der mit diesem automatisierten Fahrsystem verbundenen Vorteile (das Fahrzeug holt PassagierInnen im Nahbereich ab) wird die Entwicklung des Parkpilots nicht nur für den privaten Pkw angedacht, sondern auch vonseiten der Hersteller (z. B. BMW und Daimler) verstärkt mit Überlegungen zu den eigenen Car-Sharing-Diensten (Drive-Now und Car2go) verschränkt (Ritz 2018, S. 114; Lenz \& Fraedrich 2015, S. 185).

\section{$3 \quad$ STADT- BZW. CITYPILOT}

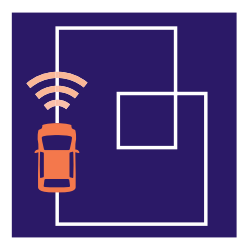

Die Fahrzeughersteller, die sich immer mehr als Mobilitätsanbieter darstellen, arbeiten jedoch - zum Teil gemeinsam mit neuen Playern am Mobilitätsmarkt wie Uber oder Waymo - auch an automatisierten Fahrsystemen für eher ,städtische“ Umfeldbedingungen bzw. definierte Bereiche im niederrangigen Straßennetz (Ritz 2018, S. 135). Aktuelle Beispiele hierfür sind die Entwicklung eines Stadtpilots durch Audi, die Tests von Waymo in einem festgelegten 100 Quadratmeilen großen Gebiet in Chandler, einem (suburbanen) Vorort von Phoenix in Arizona, oder die Ankündigung von Daimler und Bosch, in der kommenden Dekade gemeinsam Fahrzeuge des Level 4 im urbanen Umfeld auf die Straße zu bringen (Hawkins 2017; Ritz 2018, S. 30; Daimler 2018).

In diesem Fall übernimmt das häufig als Stadt- bzw. Citypilot bezeichnete automatisierte Fahrsystem in einem definierten, freigegebenen Bereich des niederrangigen Straßennetzes die Fahraufgabe. Der/Die FahrerIn wird somit in diesem Bereich zum Passagier (Wachenfeld et al. 2015, S. 17; Altenburg et al. 2018, S. 4).

Durch die Vorteile, die automatisierte Fahrsysteme in diesem Zusammenhang bringen (z. B. Möglichkeit eines Tür-zu-Tür-Service; Lenz \& Fraedrich 2015, S. 185), werden solche Systeme verstärkt auch mit Überlegungen zu Konzepten wie Car-Sharing und Ride-Sharing verschränkt und dann häufig als automatisierte Einzel- oder Sammeltaxis bezeichnet. Die Grenzen zwischen MIV und ÖV sind hierbei zunehmend fließend, da in solchen Anwendungsfällen sowohl durch Fahrzeughersteller und neue Player am Mobilitätsmarkt Geschäftsmodelle gesehen werden, gleichfalls aber auch öffentliche Verkehrsunternehmen ihr Angebot durch solche automatisierten Fahrsysteme vor allem im suburbanen Raum flexibler anbieten könnten. Grundsätzlich wird mit solchen automatisierten Fahrsystemen angestrebt, dass der Fahrgast in einem Not- fall mit der Leitstelle Kontakt aufnehmen kann und die Fahrt über das Handy bezahlt (Eckstein et al. 2018, S. 9).

Solche automatisierten Fahrsysteme werden nicht nur im Personen-, sondern auch im Güterverkehr, insbesondere als Lösungen für die letzte Meile, diskutiert. Sie sollen die letzte Meile von einem innerstädtischen Lagerhaus oder einem stationären Händler zu einem/r KundIn in einem definierten, freigegebenen Bereich im niederrangigen Straßennetz übernehmen. Als Beispiel können die häufig als Lieferroboter bezeichneten Fahrzeuge des Herstellers Starship Technologies genannt werden, die einen Behälter besitzen, in welchem eine Sendung eingelegt werden kann (Vogler et al. 2018, S. 152). Aktuell sind solche automatisierten Fahrsysteme noch meist in Büroparks (z. B. in Mountain View, USA) oder anderen Sonderarealen (wo es zum Teil schon seit längerer Zeit solche automatisierten Fahrsysteme etwa für den innerbetrieblichen Transport gibt; Flämig 2015, S. 378) in Betrieb (Hern 2018). Auch hier geht es darum, die Anforderungen an das automatisierte Fahrsystem zu reduzieren. Generell sollen solche Fahrsysteme aber in definierten, freigegebenen Bereichen auf Fußwegen mit einer Geschwindigkeit von max. $6 \mathrm{~km} / \mathrm{h}$ operieren. Sie wurden in dieser Form unter anderem bereits in Hamburg (gemeinsam mit Hermes) oder Düsseldorf getestet.

\section{AUTOMATISIERTER SHUTTLEBUS}

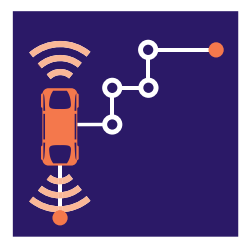

Automatisierte Shuttlebusse werden aktuell vor allem von öffentlichen Verkehrsunternehmen getestet. Beispiele hierfür sind das Testen von Shuttlebussen durch die Deutsche Bahn in Bad Birnbach, durch die Wiener Linien in Wien oder durch die PostAuto Schweiz AG in Sitten. Die bekanntesten Hersteller dieser automatisierten Shuttlebusse sind die Firmen Navya und EasyMile. Die Shuttlebusse sind meist auf eine Kapazität von 8 bis 12 Personen ausgelegt und können laut Herstellern eine Geschwindigkeit von maximal 45 km/h erreichen (Navya 2017, S. 13); derzeit operieren sie aber meist lediglich mit Geschwindigkeiten um 15 bis 20 km/h (Zankl \& Rehrl 2017, S. 38; Postauto Schweiz AG 2016).

Getestet werden die automatisierten Shuttles von den öffentlichen Verkehrsunternehmen vor allem als mögliche Zubringer für die Hauptlinien des U- und S-Bahn-Verkehrs und damit als Ergänzung des ÖV, beispielsweise im suburbanen Raum (Michelmann et al. 2017, S. 2). Der mögliche Entfall der Personalkosten sowie der flexiblere Einsatz aufgrund geringerer Größen wird dabei als eine Erleichterung für den wirtschaftlichen Betrieb des ÖV-Angebots in solchen Räumen gesehen (Lenz \& Fraedrich 2015, 
S. 191; Haider \& Klementschitz 2017, S. 7; Eckstein et al. 2018, S. 9). Es ist davon auszugehen, dass automatisierte Shuttles zunächst nur auf ausgewählten, definierten und freigegebenen sowie gekennzeichneten Routen bzw. Strecken (und Fahrbahnen) fahren und an definierten Haltestellen stehen bleiben. Sie können in diesen Bereichen ohne FahrerIn operieren, werden aber ähnlich der Luftfahrt von einer Leitwarte durch LotsInnen überwacht und gegebenenfalls manövriert werden (Eckstein et al. 2018, S. 8). Im Zuge einer fortschreitenden technischen Entwicklung könnte sich das Operationsgebiet solcher automatisierter Fahrsysteme letztlich auch auf definierte, zugelassene Bereiche im niederrangigen Straßennetz erweitern. Das automatisierte Fahrsystem des automatisierten Shuttlebusses würde sich damit dem eines Stadtpiloten annähern - verschränkt mit dem Konzept des RideSharings.

\section{ZUSAMMENFASSENDER ÜBERBLICK}

Zusammenfassend können die beschriebenen, ausgewählten automatisierten Fahrsysteme anhand ihrer Geographie bzw. Szenerie, die mehrere Parameter der ODD umfasst, vereinfacht in 1) Autobahn und autobahnähnliche Straßen, 2) Bereiche im niederrangigen Straßennetz, 3) Routen im niederrangigen Straßennetz sowie 4) Sonderareale unterteilt werden (Abb. 4.1.6). Während der Autobahnpilot ausschließlich auf Autobahnen und autobahnähnliche Straßen fokussiert, ist die Funktionstüchtigkeit des Parkpiloten (Valet-Parken) zunächst allein in Sonderarealen wie Parkhäusern gegeben. Der sogenannte Stadtpilot fo- kussiert hingegen auf definierte, freigegebene Bereiche im niederrangigen Straßennetz, während der automatisierte Shuttlebus zunächst allein auf definierten Routen im niederrangigen Straßennetz operiert. Zukünftig könnten automatisierte Fahrsysteme des Level 4 auch Kombinationen dieser Szenerien abdecken. Am Ende der technischen Entwicklung steht letztlich ein automatisiertes Fahrsystem des Level 5, dessen Funktionstüchtigkeit ODD-unspezifisch in allen Kontexten bzw. Szenerien und (weitestgehend) ohne Einschränkungen gegeben ist (Shladover 2018, S. 195).

Die hier beschriebenen automatisierten Fahrsysteme des Level 4 sind sowohl im Personen- als auch im Güterverkehr, aber nur in Teilräumen des Straßennetzes von Relevanz (Abb. 4.1.7).

Der Autobahnpilot kann dabei sowohl im privaten Pkw als auch in Logistikfahrzeugen wie Lkws und (Klein-) Transportern oder sogar bei Fernbussen eingesetzt werden. Der Parkpilot wird vor allem im Zusammenhang mit privaten Pkws oder Kleinstfahrzeugen wie etwa Pods oder LSEV (low-speed electric vehicles), aber auch im Zusammenhang mit Car-Sharing (Einzeltaxi) diskutiert. Der sogenannte Stadtpilot kann sowohl beim privaten Pkw oder bei Kleinstfahrzeugen, jedoch auch beim Car-Sharing (Einzeltaxi) und Ride-Sharing (Sammeltaxi) - hier verschwimmen die Grenzen zwischen individuellem und kollektivem Personenverkehr - sowie bei (Last-Mile-)Logistikfahrzeugen Verwendung finden. Der automatisierte Shuttlebus wird im öffentlichen Personenverkehr mit festen oder flexiblen Haltestellen sowie als Free-floatingoder routenbasiertes System thematisiert.

Abbildung 4.1.6: Ausgewählte automatisierte Fahrsysteme und ihre Operational Design Domain

\begin{tabular}{|r|l|l|l|}
\hline & \multicolumn{3}{|c|}{ OPERATIONAL DESIGN DOMAIN } \\
AUTOMATISIERTES \\
FAHRSYSTEM (ADS)
\end{tabular}




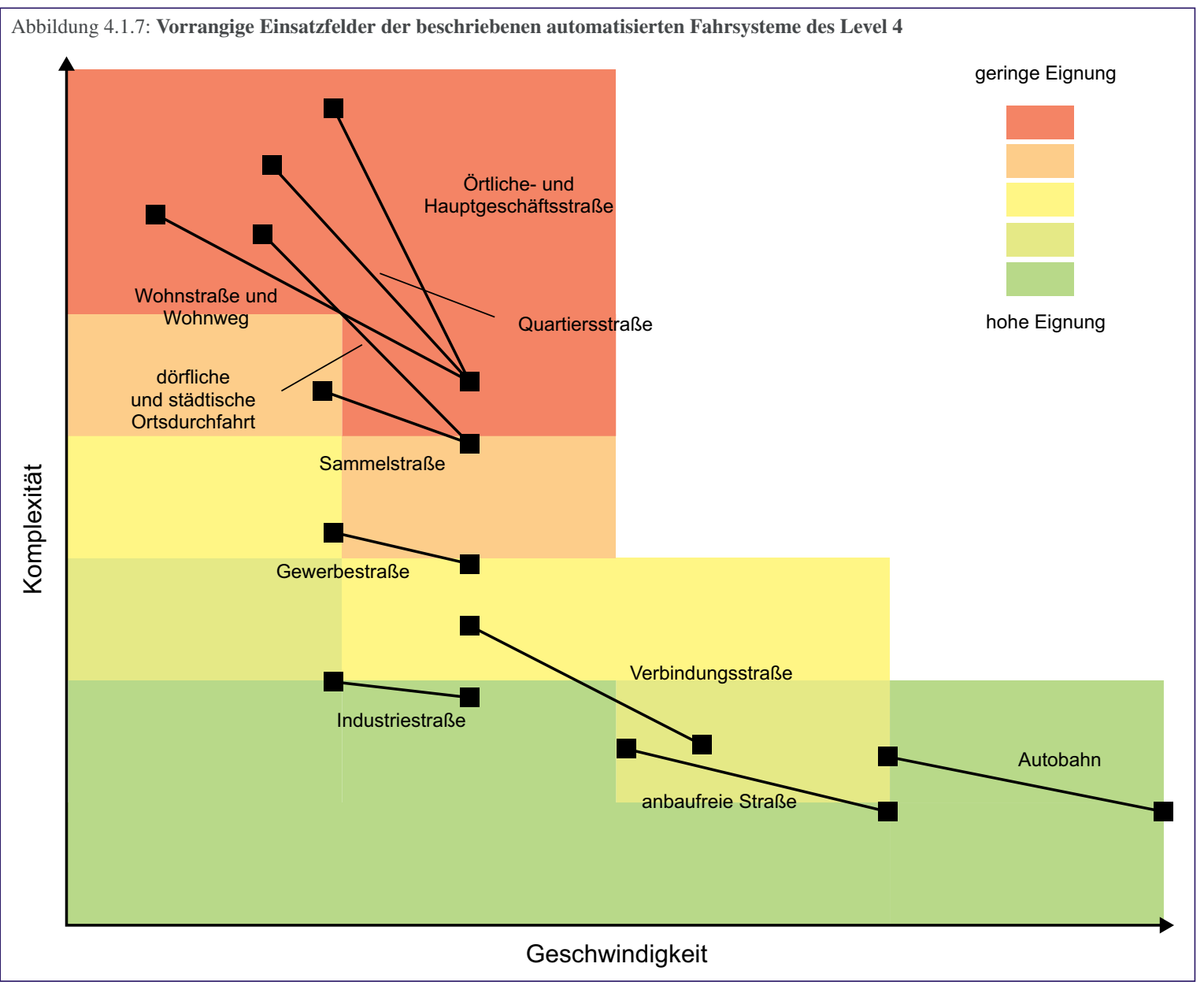

Quelle: AVENUE21

\section{DIE BEDEUTUNG DES LANGEN LEVEL 4 FÜR DIE STADT- UND MOBILITÄTSPLANUNG}

Der Begriff der ODD wurde von SAE erst ab dem Jahr 2016 eingeführt und gewinnt seither an Signifikanz: sowohl in der Technologieentwicklung als auch in der Stadt- und Mobilitätsplanung. Eine ausgedehnte Übergangszeit, in der automatisierte Fahrzeuge möglich sind und auch zum Einsatz kommen, aber dies nur innerhalb bestimmter Umfeldbedingungen, hat eine zeitliche Staffelung zur Folge. Diese Staffelung ergibt sich aus der Komplexität, die gerade urbane Straßenräume ausmacht. Die Ansicht, dass avF homogen in Städten eingesetzt werden, ist damit überholt. Vielmehr werden unterschiedliche Anwendungen in europäischen Städten räumlich selektiv eingesetzt werden und damit auch nur bestimmten Bevölkerungsgruppen zur Verfügung stehen. Weiters wäre ein mögliches Verschieben der Standortqualitäten die Folge, wenn nur einige Standorte an ein überregionales Verkehrsnetz angeschlossen sind und andere nicht. Die möglichen Veränderungen durch automatisierte Fahrzeuge in der Stadt finden somit zunächst nur an verstreuten Stellen statt (Ritz 2018, S. 74). Kapitel 4.4 behandelt dieses Thema im Detail. Wie in den Szenarien in Kapitel 5 gezeigt wird, erhöht sich durch diese Einschränkung sowohl der Handlungsbedarf als auch der Handlungsspielraum für AkteurInnen der Stadt- und Mobilitätsplanung.

\section{DIE VERNETZUNG DER FAHRZEUGE}

Im Zusammenhang mit der Automatisierung der Fahrzeuge spielt auch deren Vernetzung untereinander oder mit der Umwelt eine immer größere Rolle (Rammler 2016, S. 14; Bönninger et al. 2018, S. 97). Letztlich bildet die Vernetzung in den meisten der vorher beschriebenen Einsatzfeldern - insbesondere beim Car- und Ride-Sharing - die Voraussetzung für den Zugriff auf das automatisierte Fahrzeug per App oder Webportal durch die FahrzeugnutzerInnen (Johannig \& Mildner 2015, S. 4).

Die Kapazität von Fahrzeugen, sich zu vernetzen, wird nach dem Gegenüber der Vernetzung gegliedert. ,Vehicle to Everything " oder V2X (die Summe aller Funktionen in Abb. 4.1.8) beschreibt den Zustand vollständiger Kommunikationsfähigkeit von Fahrzeugen: sei es untereinander (V2V), mit der Infrastruktur (V2I) oder mit mobilen Endgeräten von FußgängerInnen (V2P; Shladover 2018, S. 191). 
Abbildung 4.1.8: Überblick über Vernetzungstypen und ihre möglichen Anwendungen

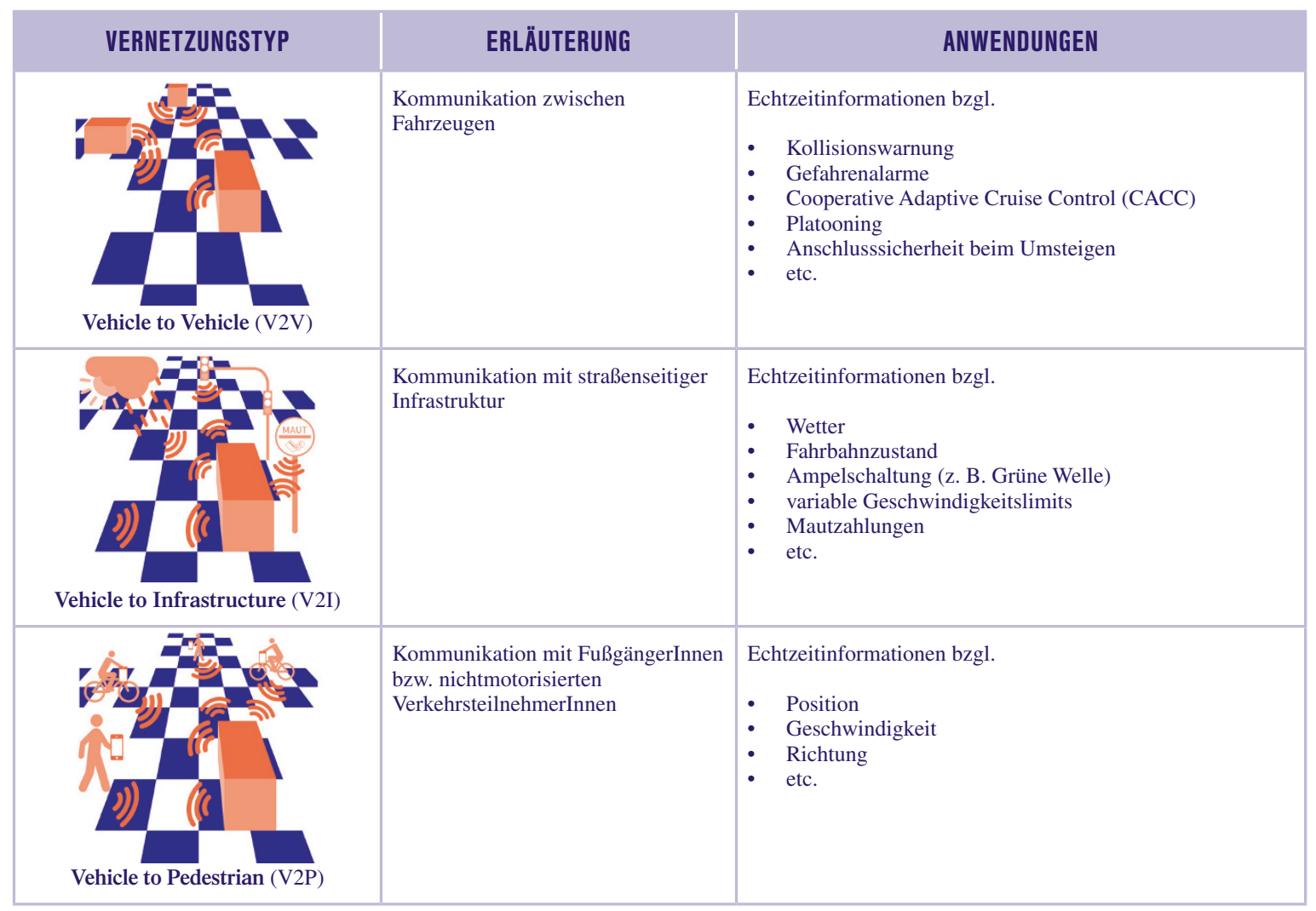

Quelle: AVENUE21 nach Shladover (2018, S. 191) und Perret et al. (2017, S. 16)

In der Anwendung reichen die Möglichkeiten von dynamischen und hoch verdichteten (Güter-)Platoons sowie vernetzten Kollisionswarnungen und Gefahrenalarmen (V2V) über Echtzeitinformationen $\mathrm{zu}$ Wetter- und Fahrbahnzustand (V2I) sowie Shuttles, die ihren Fahrstatus direkt an FußgängerInnen oder vorausschauend an mobile Endgeräte von FußgängerInnen kommunizieren (V2P; Owens et. al 2018, S. 71; Shladover 2018, S. 191). Darüber hinaus sollen NutzerInnen die Möglichkeit haben, von Abfahrt bis Ankunft schnittstellenlos und situationsabhängig mobil zu sein (Boban et. al 2017, S. 2). Abbildung 4.1.8 gibt einen Überblick über die unterschiedlichen Vernetzungstypen und mögliche Anwendungen.

Voraussetzung dafür ist eine verlässliche und stabile sowie vor allem eine leistungsstarke und schnelle Informations- bzw. Datenübertragung auf Basis von Kommunikationstechnologien, Sensoren und Netzwerkverbindungen (Maracke 2017, S. 64). Aufseiten der Industrie wird dafür zwischen kurzen und langen Latenzzeiten unterschieden. Während Erstere vor allem Kollisionswarnungen, Geschwindigkeitsbegrenzungen oder elektronischen Park- und Mautzahlungen dienen, betreffen Letztere vor allem Infotainment und Verkehrsinformationsleistungen bei Langstrecken.

Für die Datenübertragung können verschiedene drahtlose Kommunikationstechnologien zum Einsatz kom- men (Shladover 2018, S. 192). Primär anwendbare Technologien sind hierbei ITS-G5 (WLAN IEEE 802.11p), der zellulare Mobilfunk (LTE-Vehicular/ LTE Advanced oder zukünftig 5G) sowie das digitale Broadcasting wie z. B. DAB (Digital Audio Broadcasting), DAB+, DMB (Digital Multimedia Broadcasting) oder DAB-IP. Derzeit ist noch vollkommen offen, welche Kommunikationstechnologie sich in Zukunft im Zusammenhang mit dem automatisierten Fahren durchsetzen wird: Während die Europäische Kommission das Konzept eines komplementären Kommunikationsmixes mit dem Einsatz hybrider Kommunikationstechnologien verfolgt, wird in den USA durch die Behörde für Straßen- und Fahrzeugsicherheit in einem Gesetzesentwurf der Standard ITS-G5 für die (Nahbereichs-)Kommunikation favorisiert (Sänn et al. 2017, S. 62).

Durch die zunehmende Bedeutung der Vernetzung steigen auch die Sicherheits- und Datenschutzanforderungen (Lemmer 2015, S. 61). Jedes vernetzte Fahrzeug sammelt zahlreiche, zum Teil sensible Daten und Informationen über Bewegungsmuster, persönliche Fahrgewohnheiten oder Finanzdaten, die nicht nur gespeichert, sondern auch analysiert und abgesichert werden müssen. Ohne Sicherheitsstandards werden Fahrzeugsysteme mit zunehmender Vernetzung und Automatisierung anfälliger für Angriffe von außen, aber auch für einen Funktionsausfall (Seider \& Schmitz 2017). 
Hinsichtlich der Kommunikation von Fahrzeug zu Fahrzeug oder zwischen Fahrzeug und den Servern des Herstellers muss deshalb darauf geachtet werden, dass diese vor Hackerangriffen sicher sind, die Datenintegrität gewährleistet und die Kommunikation robust ist. Im Weiteren ist hier relevant, dass sich die Systeme nicht einfach durch Überlastungsangriffe (z. B. DDoS

- Distributed Denial of Service) unterbinden lassen (Ritz 2018, S. 205). Letztlich ist das Thema Vernetzung - insbesondere vor dem Hintergrund der Nutzerakzeptanz - eng verknüpft mit der Gewährleistung des Schutzes vor Cyberangriffen und der Vertraulichkeit der Daten (Seider \& Schmitz 2017 und Kap. 3.4).

\section{DIE BEDEUTUNG DER VERNETZUNG FÜR DIE STADT- UND MOBILITÄTSPLANUNG}

Für Städte ist die Frage der Vernetzung eine bedeutende. Eine Vernetzung mit der Infrastruktur (Ampeln, Verkehrsinformations- oder Leitsystem) bedeutet einen hohen finanziellen Aufwand (Mitteregger et al. 2019). Für die Organisation und den Zugriff auf multimodale Mobilitätsservices ist die Vernetzung von Fahrzeugen, aber auch von Flotten unterschiedlicher Betreiber entscheidend. Wollen Städte eine Rolle in der Organisation von Sharing-Angeboten spielen, ist der Zugriff auf Daten entscheidend. Auch für Verkehrsinformationssysteme sowie die Steuerung des fließenden Verkehrs durch Mautsysteme werden Daten in Zukunft von wachsender Bedeutung sein. Diese Daten können wiederum durch Investitionen in digitale Infrastruktur selbst erzeugt werden oder direkt von den Betreibern von Sharing-Flotten eingefordert werden (Kap. 3.3). 
4.2

\section{SIEDLUNGS- UND INFRASTRUKTURELLE ASPEKTE EINER RÄUMLICH SELEKTIVEN DURCHSETZUNG}

Die Entwicklung von Siedlungen und Städten ist eng mit dem Verkehr bzw. mit der Entwicklung neuer technologischer Mobilitätsinnovationen verbunden. Wurden bis Mitte des 19. Jahrhunderts Wege fast ausschließlich zu Fuß unternommen, bildeten wenig später Pferdekutschen und -busse, Eisenbahnen, Straßen- und U-Bahnen sowie Autos den Verkehr und prägten dementsprechend die Siedlungsentwicklung (Abb. 4.2.1; Safdie \& Kohn 1998, S. xii). Heute lässt sich ein Zusammenhang von Mobilitätstechnologien und der Entwicklung der europäischen Städte, ihrer Vielfalt aus historischen, mittelalterlichen Stadtvierteln und neueren Stadtquartieren herstellen. ,So spiegelt die Entwicklung der Siedlungsstruktur - das heißt der Ausdehnung, des inneren Gefüges und der Verteilung der Siedlungen im Raum - die geschichtliche Entwicklung der Verkehrssysteme: der zur Verfügung stehenden Verkehrsmittel, der anzutreffenden Verkehrswege und vor allem der Verkehrsgeschwindigkeiten“ (Schmitz 2001, S. 27).

Bei Mobilitätsinnovationen, die das Leben und die Gestalt der Siedlungen geprägt haben, waren sowohl Fahrzeuge als auch Verkehrsinfrastruktur (Schienen- und Straßennetze, Häfen und Flughäfen) ausschlaggebend. Auch die Geschichte der Infrastruktur ist von Innovationen geprägt, die häufig unerwähnt bleiben (McShane 1994). Die Infrastruktur stellt jenen unbeweglichen Teil dar, der für die räumliche Wirkung neuer Verkehrsmittel
(Erreichbarkeitsveränderung, Wandel von Raumnutzung und damit verbundene ökonomische, soziale Effekte) wesentlich ist. Häufig ist es (in Europa) die öffentliche Hand, die finanzielle Mittel für den Bau und die Wartung der Verkehrsinfrastruktur bereitstellt. Die volkswirtschaftliche Sinnhaftigkeit von Investitionen in Verkehrsinfrastruktur ist daher ein viel diskutiertes verkehrspolitisches Thema (Aschauer 1989, Deng 2013). Dieser Aspekt wird durch die Betrachtung von avF im SAE-Level 4, die nur in Teilen des Straßennetzes eingesetzt werden können und dadurch infrastrukturellen Investitions- bzw. Ausbaubedarf auslösen könnten, in den Mittelpunkt gerückt.

\subsubsection{SIEDLUNGSENTWICKLUNG UND MOBILITÄTS- INNOVATIONEN: EIN BLICK IN DIE VERGANGENHEIT}

Im Mittelalter waren europäische Städte meist durch eine hohe Dichte, Enge und Gedrängtheit geprägt. Dies resultierte aus der Notwendigkeit von Befestigungsanlagen um die Stadt, bedingt durch die relative räumliche Nähe von Städten zueinander, und aus dem Umstand, dass die meisten Wege zu Fuß unternommen werden mussten (Mumford 1984; Wegener \& Fürst 1999, S. 4). Das Fußgängernetz war in weiterer Folge bis ins 19. Jahrhundert das die Stadt-

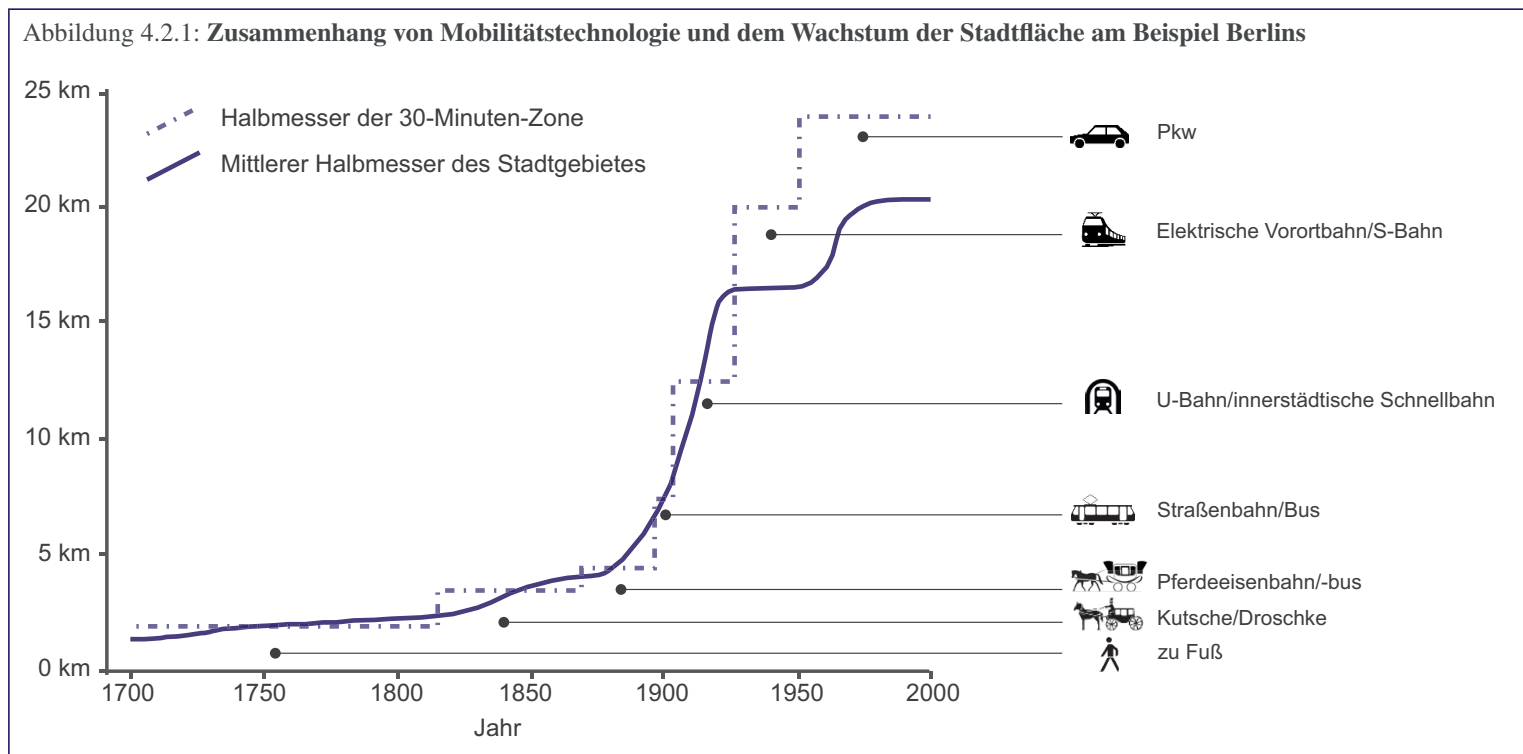

Quelle: AVENUE21 nach Kagermeier (1997, S. 25) und Lehner (1964, S. 22-23) 
struktur bestimmende Verkehrssystem. Die Struktur dieser Städte war dementsprechend auf fußläufige Erreichbarkeit ausgerichtet: Städte waren kleine und geschlossene Stadtkörper und der Durchmesser des Stadtgebiets betrug nicht viel mehr als fünf Kilometer (Kainrath 1997, S. 16). Solche Strukturen findet man auch heute noch in den mittelalterlichen Kernen zahlreicher europäischer Städte (Newman \& Kenworthy 1999, S. 28). Mit dem Aufkommen von Pferdebussen, Straßenbahnen, überregionalen Eisenbahnen sowie Holz- und Eisenschienen und neuen Fahrbahndecken (Stein-, Ziegel- und Holzpflasterungen, Makadam und schließlich Asphalt) im 18. Jahrhundert kam es zu Veränderungen in der Erreichbarkeit und in weiterer Folge auch der Siedlungsstruktur (Kainrath 1997, S. 16). Ein grundlegender Wandel der gesellschaftlichen Bedeutung von Mobilität und Straßenraum fand statt (McShane 1979, S. 57-80).

Die letzte weitreichende Veränderung von Mobilität brachte die Verbreitung des privaten Automobils im Laufe des 20. Jahrhunderts zunächst in den USA (wo sich auch Asphaltdecken schneller durchsetzten), nach dem Zweiten Weltkrieg dann auch in Europa. Das Auto ermöglichte durch die flächenhafte Erreichbarkeit nun auch die Nutzung der Gebiete zwischen den Eisenbahnachsen für die Stadterweiterung (Wegener \& Fürst 1999, S. 5). Zudem führte die mit dem Wirtschaftswachstum verbundene allgemeine Wohlstandsentwicklung nach dem Ende des Zweiten Weltkrieges in Europa zu einer generellen individuel- len Motorisierung. Die weite Verbreitung des privaten Autos ermöglichte und förderte gleichzeitig die räumliche Trennung von Funktionen wie etwa Wohnen und Arbeiten (Kagermeier 1997, S. 24). Die Folge war ein weniger geordnetes und stärker disperses Stadtwachstum mit dem Phänomen einer starken Zersiedelung am Stadtrand (Wegener \& Fürst 1999, S. 5). Am Standrand dominiert heute das Auto, da im öffentlichen Verkehr erhebliche Lücken bestehen (Kainrath 1997, S. 16).

\subsubsection{ZEITLICHKEIT DES WANDELS: DIE DIFFUSION VON TRANSPORTTECHNOLOGIEN}

Bevor Transporttechnologien räumlich wirksam werden, müssen sie von einer stetig wachsenden Zahl an Mitgliedern angenommen und im Alltag genutzt werden. Dies sind langfristige Prozesse, ein Umstand, der durch die Theorie über disruptive Technologien (Christensen 2003) häufig übersehen wird (King \& Baatartogtokh 2015). Die Diffusion von Innovationen (Rogers 2003) ist ein kommunikativer Prozess, währenddessen sich Individuen bzw. soziale Gruppen für die Adaptierung und den damit verbundenen Aufwand entscheiden. Die Diffusion beginnt langsam, nimmt Fahrt auf, um dann wieder an Geschwindigkeit zu verlieren, sobald Sättigungseffekte auftreten. Dies hat sich als robuste Erkenntnis erwiesen (Kucharavy \& De Guio 2011, Grubler et al. 2016).

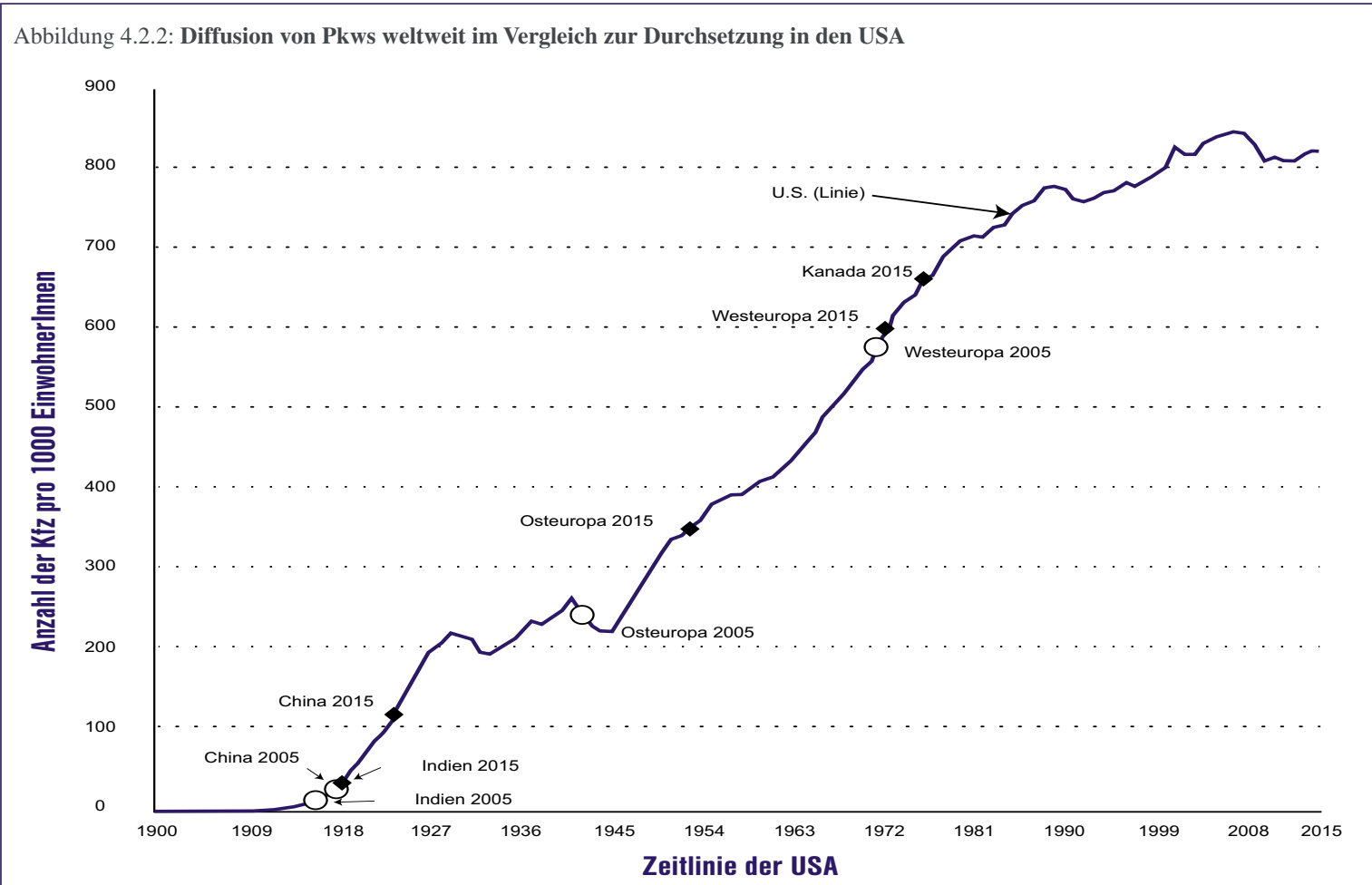

Quelle: Davis et al (2015, S. 3-8) 
Die Dauer von technologischen Diffusionsprozessen ist von einer Reihe von Faktoren abhängig. Dazu zählt die Komplexität der Technologie, die Länge der „formativen Phase“ (Bento \& Wilson 2016), welcher Aufwand durch die Aufhebung bestehender Praktiken besteht, wie hoch die Investitionen zur Einführung ausfallen, welche Vorteile für unterschiedliche Akteursgruppen erwartet werden und ob begleitende Anpassungen in Gesetzen und Verordnungen notwendig werden.

In Abbildung 4.2.3 werden die von Grubler et al. (2016) beschriebenen Merkmale langer Diffusionsprozesse zusammengefasst und auf die Charakteristiken des avV angewendet. Der hohe globale Durchsetzungsgrad des Straßenverkehrs und die Vielzahl an Individuen, die von den Änderungen des Verkehrs und im Straßenraum betroffen sein werden, sind wesentliche Faktoren für die Dauer des Diffusionsprozesses des avV. Hinzu kommt ein hoher Abstimmungsbedarf unterschiedlicher politischer Ebenen und StakeholderInnen (s. Kap. 4.6), z. B. in Fragen der Zertifizierung von diversen Funktionen automatisierter Fahrsysteme und Fahrzeugtypen (Walker 2016). Hinzu kommt, dass Standards für die physische und digitale Infrastruktur und zur Sicherung bestimmter ODD koordiniert und durchgesetzt werden müssen (European Commission 2017, 2019). Außerdem sind bei Investitionen auf unterschiedlichen Ebenen des Straßenverkehrsnetzes erhebliche Kosten zu erwarten, die Staaten und Kommunen vor große Herausforderungen stellen (POLIS 2018, Mitteregger et al. 2019). Aufseiten der technologischen Entwicklung ist das Zusammenführen unter- schiedlicher Sensorendaten zu einem kohärenten Bild eine zentrale Herausforderung, die bisher in diesem Umfang in keinem anderen Anwendungsfall geleistet werden musste (s. Kap. 4.4).

\section{FRAGEN DER AKZEPTANZ AUTOMATISIERTER FAHRSYSTEME}

Die Antwort auf die Frage nach der Akzeptanz der avF wird von der Dauer des Diffusionsprozesses bestimmt. Der betrifft nicht nur die Beförderten (PassagierInnen), sondern auch alle anderen Verkehrsteilnehmenden. Wesentlich ist, dass mit den „Fahrrobotern“ des Level 4 Menschen zum ersten Mal in kritischen (und potenziell tödlichen) Situationen mit automatisierten, mobilen Maschinen im öffentlichen Raum interagieren müssen. Bis heute ist diese Erfahrung spezialisierten Berufsgruppen wie der Logistik (z. B. in Häfen oder Logistikcentern), der Landwirtschaft, dem Bergbau oder dem Militär (Drohnen) vorbehalten. Abbildung 4.2.4 fasst die wesentlichen, heute diskutierten Bereiche der Akzeptanz zusammen.

PassagierInnen von avF müssen lernen, zu akzeptieren, dass sie von einer softwaregesteuerten Maschine gefahren werden, welche die Handlungen während der Fahrt umfassend steuert, überwacht und möglicherweise durch externe Instanzen (Polizei, Mobilitätsanbieter, Infrastrukturbetreibende, aber auch Hacker) beeinflusst bzw. gelenkt werden kann. Die Interaktion mit dem Fahrzeug als PassagierIn stellt für bestimmte soziale Gruppen eine hohe Hürde dar (Vertrauen, Technikaffinität, ,digital divide“). Aber auch für andere Verkehrs-

Abbildung 4.2.3: Gegenüberstellung der Merkmale langer Technologiediffusionen und Charakteristiken automatisierter und vernetzter Fahrsysteme

\begin{tabular}{|c|c|}
\hline MERKMALE & CHARAKTERISTIKEN \\
\hline $\begin{array}{l}\text { Anpassungen mehrerer Technolo- } \\
\text { gien, im organisatorischen und } \\
\text { institutionellen Umfeld sowie bei } \\
\text { Infrastrukturen werden notwen- } \\
\text { dig. }\end{array}$ & $\begin{array}{l}\text { - Institutionen und Organisationen müssen erst den legislativen Rahmen für den avV schaffen bzw. die } \\
\text { Freigabe oder Zertifizierung entwickeln (Schoitsch et al. 2016). Durch transnationale Verkehrsnetze } \\
\text { bzw. die standardisierte Produktion von Fahrzeugen besteht großer Abstimmungsbedarf auf allen } \\
\text { politischen Ebenen. } \\
\text { - Automatisierte und vernetzte Fahrzeuge ersetzen nicht- oder gering automatisierte Fahrzeuge in } \\
\text { bereits existierender Infrastruktur. Auch wenn ein Teil der bestehenden Infrastruktur genutzt werden } \\
\text { kann, erzeugt es doch einen hohen Anpassungsbedarf. }\end{array}$ \\
\hline $\begin{array}{l}\text { Neue technologische und soziale } \\
\text { Konzepte müssen entwickelt bzw. } \\
\text { erlernt werden. }\end{array}$ & $\begin{array}{l}\text { Die zentrale technische Herausforderung des avV ist das Zusammenführen unterschiedlicher } \\
\text { Sensordaten zu einem kohärenten Bild. Dieses Bild muss maschinell (durch künstliche Intelligenz) } \\
\text { interpretiert werden und dient als Basis für Verkehrslenkung und Fahrentscheidungen (s. Kap. 4.6). } \\
\text { - Die Interaktion von nichtmenschlichen Akteuren und anderen Verkehrsteilnehmenden stellt neue } \\
\text { sozialpsychologische Herausforderungen dar, die langfristig erlernt und akzeptiert werden muss } \\
\text { (Merat et al. 2017, Rogers 2003). }\end{array}$ \\
\hline $\begin{array}{l}\text { Hohe Investments in weit verbrei- } \\
\text { tete Technologien und Infrastruk- } \\
\text { turen werden notwendig, wodurch } \\
\text { der Aufwand der Adaption erst zu } \\
\text { einem späteren Zeitpunkt rentabel } \\
\text { wird. }\end{array}$ & $\begin{array}{l}\text { - Investitionen könnten auf allen Ebenen des Verkehrsnetzes notwendig werden. Relevant sind } \\
\text { die Netzgrößen von Autobahnen und Schnellstraßen (1,9\% des Verkehrsnetzes in Österreich), } \\
\text { Landesstraßen (29,3\%) und Gemeindestraßen (68,73\%; BMVIT 2018) und die jeweils } \\
\text { unterschiedlichen Akteure, die mit Wartung und Instandhaltung betraut sind, sowie unterschiedliche } \\
\text { Arten der Finanzierung (z. B. durch Maut- oder Transferzahlungen). } \\
\text { - Darüber hinaus könnten Investitionen in die digitale Infrastruktur, die Vernetzung der Fahrzeuge } \\
\text { hinzukommen (DG MOVE 2016, S. 41). Das Ausmaß der Investitionen ist räumlich sehr } \\
\text { unterschiedlich und gegenwärtig kaum abzusehen. }\end{array}$ \\
\hline
\end{tabular}


teilnehmende, die ihr Verhalten im öffentlichen Raum mit dem des automatisierten Fahrsystems abstimmen müssen, gibt es einen hohen Bedarf, Vertrauen herzustellen (was im Rahmen aktueller Tests durch Signale an den Fahrzeugen geregelt wird). Im Fall von avRide-Sharing wird zudem der relativ kleine Raum des Fahrzeugs mit unbekannten Menschen für die Dauer der Fahrzeit ohne die Anwesenheit eines/r FahrerIn geteilt - eine Herausforderung, die vor dem Hintergrund zunehmender gesellschaftlicher Ausdifferenzierung dazu führen wird, dass die Akzeptanz vor allem in dünn besiedelten Räumen und zu Schwachlastzeiten zu Beginn gering ist und gegebenenfalls sinkt (Merat et al. 2017). Das gilt im besonderen Maße gerade für mobilitätseingeschränkte Gruppen, die von diesen Shuttlediensten profitieren sollten.

Eine geringe Akzeptanz automatisierter Fahrzeuge kann dazu führen, dass sich das Verhalten im öffentlichen Raum der Straße erneut grundlegend wandelt (wie dies im Fall der Automobilität bereits der Fall war, s. Kap. 3.2). Automatisierte Fahrsysteme werden im Betrieb umfassend Daten im öffentlichen Raum generieren - das Verhalten von Personen im Straßenraum eingeschlossen - und diese im Zuge datenbasierter Geschäftsmodelle oder des Verkehrsmanagements verwerten. Eine umfassende Überwachung wird aus heutiger Sicht in europäischen Städten (im Gegensatz zu chine- sischen) kaum akzeptiert. In engem Zusammenhang mit der Einführung von avF steht der Wandel von passiven zu aktiven Sicherheitssystemen, die das Verständnis des öffentlichen Raums der Straße in europäischen Städten künftig erheblich wandeln (Mitteregger 2019).

\subsubsection{DIE BEDEUTUNG DER VERKEHRS- INFRASTRUKTUR WÄHREND DES LANGEN LEVEL 4}

Der Verkehrsinfrastruktur, hier im Besonderen der StraBeninfrastruktur, wird eine zentrale Rolle für die wirtschaftliche Entwicklung von Regionen beigemessen (Aschauer 1989). Dementsprechend wird die Planung und der Bau von Verkehrsinfrastruktur als zentrale strukturpolitische Maßnahme angesehen. Die Europäische Union hat für die ,Vollendung und Modernisierung eines echten transeuropäischen Netzes" bis 2020 rund 600 Mrd. Euro beiseitegestellt (Europäische Kommission 2005, S. 3). Die europäische Initiative „Kooperative Intelligente Verkehrssysteme“ (Cooperative Intelligent Transport Systems - C-ITS) wird aus strukturpolitischer Sicht als eine der Schlüsselmaßnahmen, die avM auf den Weg bringen soll, angesehen. Sie schließt ein breites Spektrum an infrastrukturellen Anwendungen (Verkehrsleit- und -managementsysteme bis zur flächendeckenden Verfügbarkeit von 5G) ein. C-ITS sollen

Abbildung 4.2.4: In der Literatur diskutierte Kriterien der Akzeptanz durch PassagierInnen und andere Verkehrsteilnehmende des SAE-Level 4

\begin{tabular}{|c|c|}
\hline KRITERIUM & STUDIEN \\
\hline \multicolumn{2}{|r|}{ PASSAGIER/IN } \\
\hline „Das Befördertwerden“6 & Hancock et al. 2011, Malodia \& Singla 2016, Schaefer \& Straub 2016 \\
\hline Ride-Sharing & $\begin{array}{l}\text { Ahmadpour et al. 2016, Beirao \& Sarsfield-Cabral 2007, Chan \& Shaheen 2012, Dueker et al. 1977, } \\
\text { Malodia \& Singla 2016, Merat et al. 2017, Thompson et al. 1991, Venkatesh et al. } 2003\end{array}$ \\
\hline $\begin{array}{l}\text { Überwachung } \\
\text { während der Fahrt }\end{array}$ & Crittenden 2017, Litman 2017, Schulz \& Gilbert 1996 \\
\hline $\begin{array}{l}\text { Human Machine } \\
\text { Interaction }\end{array}$ & $\begin{array}{l}\text { Grush et al. 2016, Hoff \& Bashir 2015, Merat et al. 2017, Schaefer \& Straub 2016, Seppelt \& Lee 2007, } \\
\text { Venkatesh et al. 2003, Wiseman } 2017\end{array}$ \\
\hline $\begin{array}{r}\text { Zugriff } \\
\text { externer Instanzen }\end{array}$ & Anderson et al. 2016, Gontar et al. 2017 \\
\hline \multicolumn{2}{|r|}{ ANDERE VERKEHRSTEILNEHMENDE } \\
\hline Leerfahrten & Elliot \& Long 2016 \\
\hline $\begin{array}{r}\text { Human Maschine } \\
\text { Interaction }\end{array}$ & $\begin{array}{l}\text { Anderson et al. 2016, Grush et al. 2016, Hoff \& Bashir 2015, Merat et al. 2017, Parkin et al. 2016, } \\
\text { Rodriguez et al. 2016, Schaefer \& Straub 2016, Seppelt and Lee 2007, Venkatesh et al. } 2003\end{array}$ \\
\hline $\begin{array}{l}\text { Überwachung } \\
\text { auf öffentlichen Straßen }\end{array}$ & Anderson et al. 2016, Cirittenden 2017, Schulz \& Gilbert 1996 \\
\hline
\end{tabular}


die Straßensicherheit erhöhen, deren Effizienz steigern und den Komfort verbessern (Europäische Kommission 2016, S. 3). Die weitverbreitete Ansicht in Politik und Planung, dass „Straßen zu Wohlstand führen“ (Deng 2014, S. 687), ist vor allem in Europa und vor dem Hintergrund des avV differenziert zu sehen.

\section{SIEDLUNGSENTWICKLUNG DURCH VERKEHRSINFRASTRUKTUREN}

Der Zusammenhang von Effekten der Verkehrsinfrastruktur auf die Raumnutzung bzw. Siedlungsstruktur wurde in jüngeren Übersichtsstudien über unterschiedliche räumliche Maßstäbe bestätigt (Deng 2014, Kasraian et al. 2016). Er zeigt sich allerdings differenzierter als meist angenommen (Abb. 4.2.5). Es existiert ein nachweisbarer Zusammenhang zwischen dem Entwicklungsgrad des Siedlungsgebiets, dessen aktueller Erreichbarkeit und der Wirksamkeit von Verkehrsinfrastrukturinvestitionen als strukturpolitische Maßnahme. Sättigungseffekte werden deutlich, die in Gebieten ho- her Erreichbarkeit und/oder bereits stark entwickelter Siedlungsstruktur auftreten. Folglich sind die stärksten Effekte vor allem in wenig entwickelten Gebieten zu erwarten, in denen auch die Erreichbarkeit durch eine neue Verkehrsinfrastruktur gehoben werden kann.

Ein weiteres Merkmal der Verbesserung der Erreichbarkeit durch Verkehrsinfrastrukturmaßnahmen ist, dass sie aus ökonomischer Sicht nicht allen Sektoren gleichmäßig zugutekommt (Deng 2014, S. 691-692). Verkehrsintensive Sektoren wie etwa Logistik und Bauwirtschaft profitieren ungleich stärker als z. B. die Textilindustrie (Fernald 1999, S. 628). Cantos et al. (2005) konnten auch eine ungleiche Verteilung der Erreichbarkeitswirkungen hinsichtlich der Absatzmärkte von Betrieben aufzeigen. So profitieren jene Sparten, die ihre Erzeugnisse auf nationaler bzw. transnationaler Ebene vertreiben (Industrie, produzierendes Gewerbe), während Sektoren, die regional wirtschaften (Einzelhandel, Bauwirtschaft, Dienstleistungssektor, Landwirtschaft), durch eine gesteigerte Erreichbarkeit tendenziell Gewinnrückgänge zu erwarten haben.

Abbildung 4.2.5: Räumliche Effekte im Wirkungsgefüge von Transportinfrastrukturnetzen und Landnutzungen

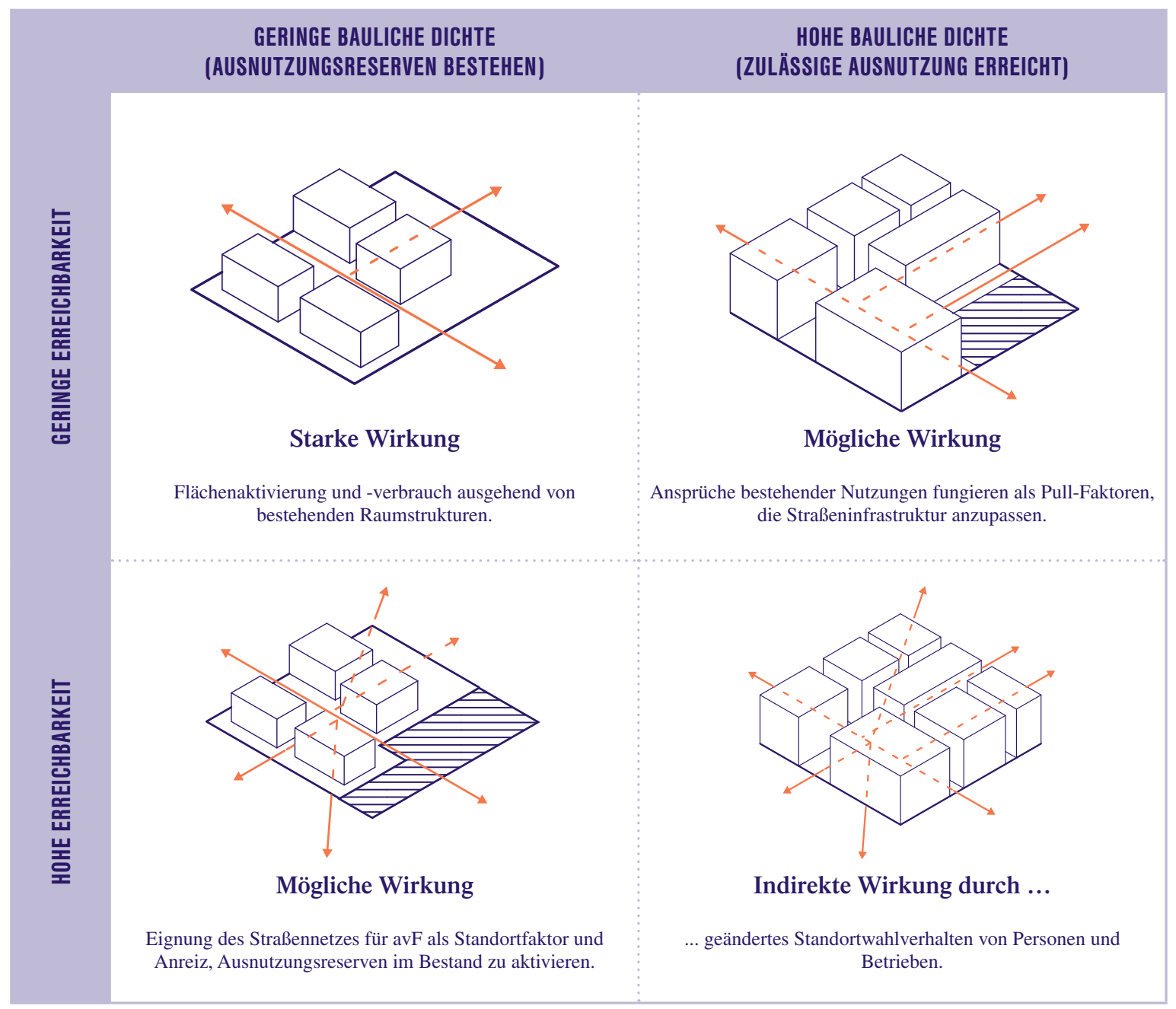

Quelle: AVENUE21 nach Kasraian et al. (2016) 
Abbildung 4.2.6: Automatisierter und vernetzter Verkehr im Wirkungsgefüge von Raumnutzung und Verkehr

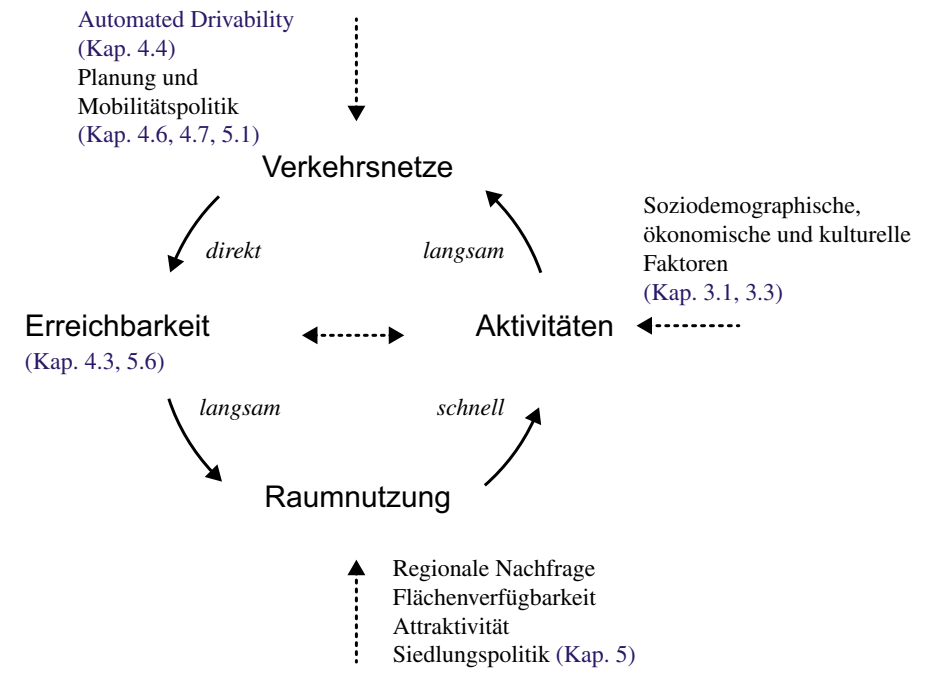

Quelle: AVENUE21 nach Wegener \& Fürst (1999) und Bertolini (2012)

\subsubsection{AVV IM WIRKUNGSGEFÜGE VON RAUM- NUTZUNG UND VERKEHR}

Raumnutzung und Verkehr stehen in einer Interdependenz zueinander und bilden über die zentrale Größe Erreichbarkeit ein Wirkungsgefüge (Abb. 4.2.6; Wegener \& Fürst 1999, S. 5-6; Bertolini 2012, S. 19). Die Wirkungen innerhalb des Systems weisen unterschiedliche Geschwindigkeiten auf: Kommt es zu einer Veränderung im Verkehrssystem, führt dies unmittelbar zu einer Veränderung der Erreichbarkeitsverhältnisse und gleichzeitig zu entsprechenden Veränderungen in der Bewertung von Distanzen (Kagermeier 1997, S. 22). Dadurch können bei veränderten Mobilitätskosten neue bzw. andere funktionale Beziehungen zwischen bereits bestehenden Standorten aufgenommen werden. Ebenso führt eine Veränderung in der Raumnutzung bzw. Siedlungsstruktur zu einer Veränderung der Erreichbarkeitsverhältnisse und zu einer relativ schnellen Adaptierung der Mobilitätsaktivitäten (innerhalb von Jahren oder sogar Tagen; Bertolini 2012, S. 2). Die jeweiligen Veränderungen in den Erreichbarkeitsverhältnissen wirken sich jedoch nur mittel- bis langfristig (in der Größenordnung von Jahrzehnten) auf die Siedlungsstruktur oder das Verkehrsangebot aus (Bertolini 2017, S. 27). So hat der veränderte Aufwand, Distanzen zu überwinden, erst mittel- bis langfristig Effekte auf die Standortentscheidungen von Bauinvestoren (und deren Bautätigkeiten) sowie auf Personen, Haushalte und Unternehmen und damit auf die Siedlungsstruktur (Kagermeier 1997, S. 22).

Automatisierte und vernetzte Fahrzeuge bergen als Mobilitätsinnovation ein hohes Potenzial zur Veränderung des Verkehrssystems. Es ermöglicht ein anderes Verkehrsangebot, das Veränderungen in der Verkehrsnachfrage mit sich bringen wird (Abb. 4.2.2; Alessandrini et al. 2015, S. 148; Friedrich \& Hartl 2016, S. 7). Lang- fristig sind Auswirkungen auf die Stadt- und Siedlungsentwicklung wahrscheinlich (Europäische Kommission 2016, S. 2). Auf Basis der Annahme eines Langen Level 4 , in dem avF nur in Teilen des Verkehrsnetzes einsetzbar sind, ist mit einer hohen Dynamik im Verkehrs- und Raumsystem zu rechnen. Vor diesem Hintergrund ist die nachstehende Betrachtung weltweiter Simulationsstudien zu sehen. 
4.3

\section{FORSCHUNGSSTAND ZU WIRKUNGEN VOLLAUTOMATISIERTER FAHRZEUGE AUF DIE STADT}

Abbildung 4.3.1: Gesammelte Ergebnisse der analysierten Simulationen aus aktuellen Studien

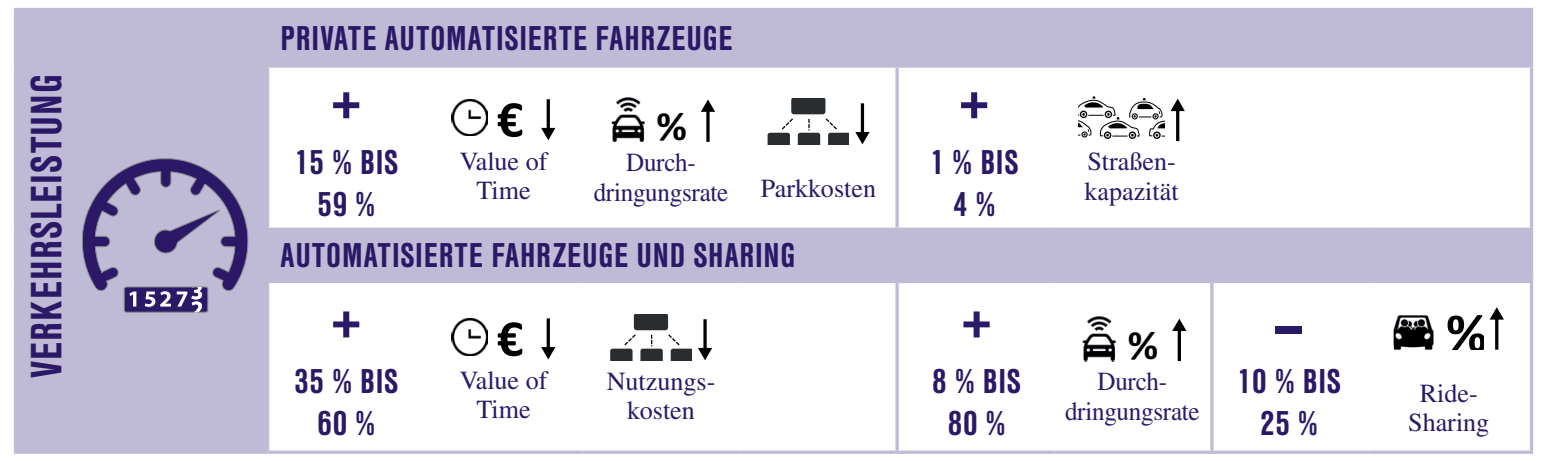

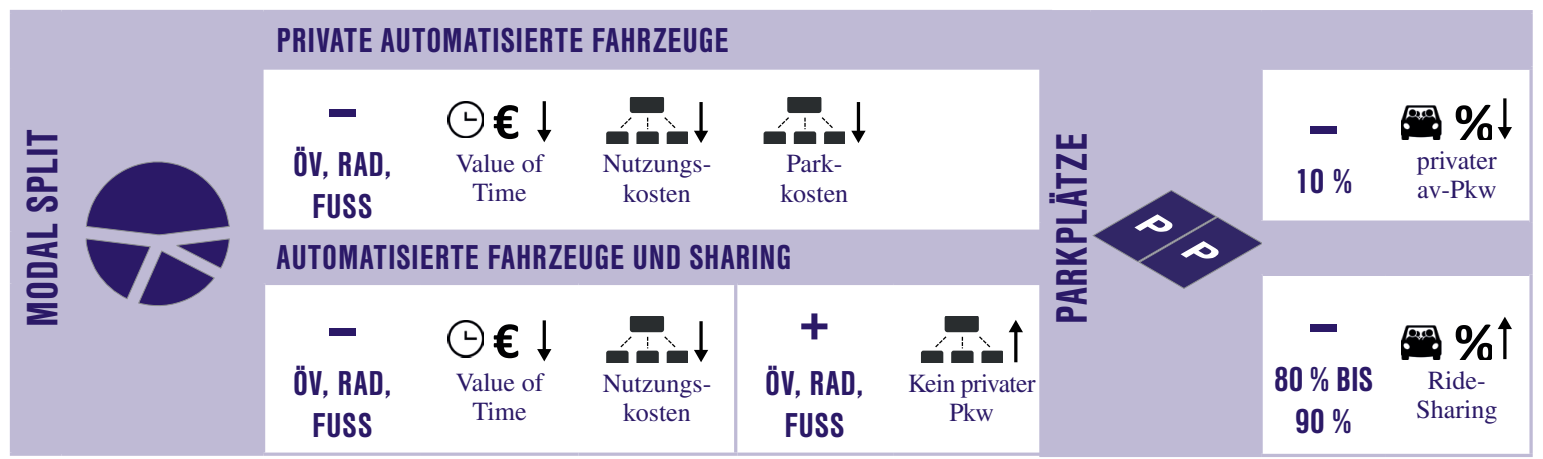

Quelle: AVENUE21

Automatisierte Fahrzeuge (Simulationsstudien untersuchen nicht die Vernetzung von Fahrzeugen, weshalb hier von automatisierten Fahrzeugen gesprochen wird) höherer Automatisierungslevels sind heute allenfalls im Rahmen von Testprojekten auf Straßen unterwegs. Um mögliche Auswirkungen automatisierter Fahrzeuge auf Städte in der Zukunft zu untersuchen und abzuschätzen, bedienen sich zahlreiche Studien daher Simulationen: Unter Verwendung von verschiedenen Annahmen und Szenarien hinsichtlich der Ausgestaltung des zukünftigen Verkehrsangebots mit automatisierten Fahrzeugen werden die möglichen verkehrlichen und räumlichen Wirkungen automatisierter Fahrzeuge computergestützt nachgebildet. Hinzu kommen Studien, die versuchen, die Wirkungen automatisierter Fahrzeuge auf Themenbereiche wie die soziale Gerechtigkeit/Inklusion oder die städtischen Budgets abzuschätzen.

Abbildung 4.3.1 und Abbildung 4.3.6 geben einen Überblick zu den Ergebnissen aktueller Studien obiger
Themenbereiche und eine kurze Erläuterung der dazugehörigen Wirkungen. Diese umfassen unter anderem Straßenkapazität/Stau, Verkehrssicherheit, Verkehrsinfrastrukturen, Fahrzeugbesitz, Verkehrsnachfrage, Parkplätze, Siedlungsstruktur, soziale Gerechtigkeit, Gesundheit, Umwelt, Cybersicherheit, Wirtschaft und Governance (Milakis et al. 2017, S. 6).

Im Rahmen des Projekts wurde eine Untersuchung zu Effekten automatisierter Fahrzeuge auf die Verkehrsnachfrage und die Siedlungsstruktur bzw. Flächennutzung durchgeführt. Insgesamt wurden somit 37 Modellierungsstudien aus verschiedenen Ländern der Welt, jedoch vor allem aus den USA und Europa (Soteropoulos et al. 2018a), analysiert. Die Ergebnisse zeigen, dass die Art des Einsatzes automatisierter Fahrzeuge sowie die mit ihnen verbundenen Modellierungsannahmen (z. B. Anteil Car- und Ride-Sharing, ,Value of Time“, Erhöhung der Straßenkapazität) dabei von besonderer Wichtigkeit sind. 


\subsubsection{VERKEHRLICHE UND RÄUMLICHE WIRKUNGEN}

Abbildung 4.3.2: Untersuchte verkehrliche und räumliche Auswirkungen von automatisiertem und vernetztem Verkehr in Simulationsstudien

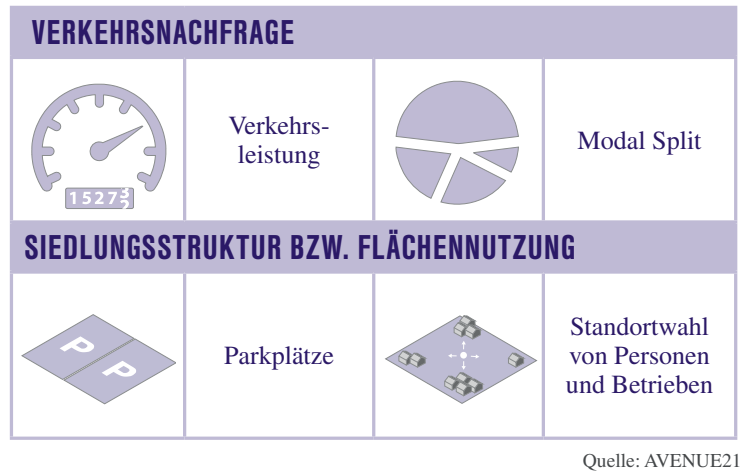

Bisherige Simulationen zeigen, dass sich vor allem vier Aspekte zu den verkehrlichen und räumlichen Wirkungen von avF unterscheiden lassen, wobei einerseits die Verkehrsnachfrage und andererseits der Einfluss auf die Siedlungsstruktur bzw. die Flächennutzung Gegenstand von Untersuchungen sind (Abb. 4.3.2). Betrachtet werden hierbei weiterführend Auswirkungen auf die Verkehrsleistung, Modal Split und Parkplätze (Abb. 4.3.1) sowie die Standortwahl von Personen und Betrieben (Abb. 4.3.3).

\section{VERKEHRSLEISTUNG}

Bezüglich der Verkehrsleistung wird deutlich, dass private automatisierte Fahrzeuge überwiegend mit einer Zunahme der gefahrenen Kilometer aufgrund von Verlagerungen von anderen Verkehrsmodi einhergehen, wobei sich die Verkehrsleistung bei Annahme einer hohen Reduktion beim Value of Time (Zeitwahrnehmung und -bewertung) sowie von Parkkosten und hohen Marktanteilen von automatisierten Fahrzeugen um $15 \%$ bis $59 \%$ erhöht. Auch mit Sharing verbundene automatisierte Fahrzeuge führen aufgrund von Verlagerungen von anderen Verkehrsmodi sowie Leerfahrten überwiegend zu einer Zunahme der gefahrenen Kilometer. Diese Zunahme liegt zwischen $35 \%$ und $60 \%$ bei der Annahme einer Reduktion beim Value of Time und geringen Kosten für die Nutzung sowie zwischen $8 \%$ und $89 \%$, wenn angenommen wird, dass ein Teil oder die gesamte Verkehrsnachfrage im MIV durch automatisierte Fahrzeuge verbunden mit Sharing abgewickelt wird. Ein Rückgang der Verkehrsleistung im Ausmaß von $10 \%$ bis $25 \%$ zeigt sich allein bei der Annahme eines hohen Anteils von Ride-Sharing.

\section{MODAL SPLIT}

Hinsichtlich des Modal Split wird ersichtlich, dass private automatisierte Fahrzeuge überwiegend zu einer Reduktion des Anteils des ÖV sowie des Fahrrads und des Zufußge- hens am Modal Split führen (s. auch Expertenbefragung in Kap. 3.4), wobei hohe Reduktionen insbesondere bei der Annahme eines hohen Rückgangs beim Value of Time sowie bei Park- und Betriebskosten ersichtlich sind. Auch automatisierte Fahrzeuge verbunden mit Sharing führen überwiegend zu einem Rückgang des Anteils des ÖV sowie des Fahrrads und des Zufußgehens am Modal Split, insbesondere wenn hohe Reduktionen beim Value of Time und geringe Kosten für die Nutzung angenommen werden. Zunahme beim Anteil des ÖV, des Fahrrads und des Zufußgehens am Modal Split zeigen sich allein bei der Annahme von eher hohen Kosten für die Nutzung und keinem Vorhandensein von privaten Fahrzeugen.

\section{PARKPLÄTZE}

Bezüglich eines möglichen Rückgangs des Parkplatzbedarfs wird deutlich, dass automatisierte Fahrzeuge verbunden mit einem hohen Anteil von Sharing, insbesondere Ride-Sharing, die Gesamtanzahl an Fahrzeugen um etwa $90 \%$ verringern könnten, was auch zu einer Verminderung von benötigten Parkplätzen im Ausmaß von $80 \%$ bis $90 \%$ führen könnte. Private automatisierte Fahrzeuge, bei denen das Sharing nur durch die jeweiligen Mitglieder des Haushalts stattfindet, könnten hingegen die Gesamtanzahl an Fahrzeugen nur um etwa $10 \%$ reduzieren. Dies hätte auch eine deutlich geminderte Verringerung von benötigten Parkplätzen und damit potenziell neu nutzbaren Flächen zur Folge.

\section{STANDORTWAHL VON PERSONEN UND BETRIEBEN}

Hinsichtlich einer Veränderung in der Standortwahl von Personen und Betrieben (Abb. 4.3.3) zeigt sich, dass private automatisierte Fahrzeuge - insbesondere wenn von einer Verringerung des Wertes der Zeit im Fahrzeug und Kapazitätssteigerungen ausgegangen wird - zu einer $\mathrm{Zu}$ nahme der Bevölkerungszahl in gut angebundenen suburbanen und ländlichen Gebieten, also zu einem tendenziell dispersen Stadtwachstum führen.

Ein effizienterer ÖV durch Automatisierung (z. B. automatisierte und vernetzte Shuttles für die letzte Meile) führt hingegen zu einer Zunahme der Bevölkerungszahl in städtischen Gebieten und damit tendenziell zu einer Begünstigung von Urbanisierungsprozessen.

Automatisierte Fahrzeuge mit Sharing könnten ebenso Zersiedelung und Suburbanisierungsprozesse dämpfen, wobei sich auch zeigt, dass sich manche Bevölkerungsgruppen aufgrund der verbesserten Erreichbarkeit weiter entfernt vom Stadtzentrum ansiedeln könnten. Automatisierte Fahrzeuge mit Sharing könnten überdies zu einem weiteren Deindustrialisierungstrend in Städten, d. h. zu einer weiteren Standortverschiebung von Betrieben aus dem sekundären Sektor in Gebiete außerhalb der Stadt, beitragen. 


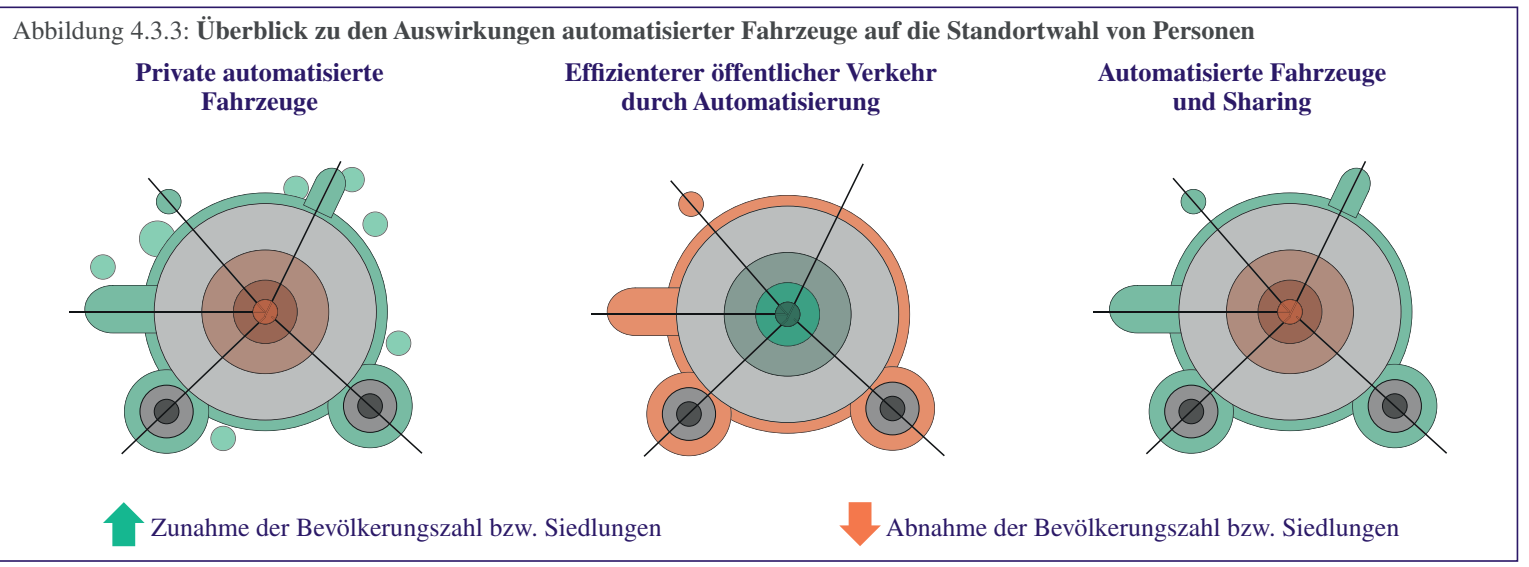

Quelle: AVENUE21 nach Kainrath (1997)

Wie bereits erwähnt, sind die verkehrlichen und räumlichen Wirkungen automatisierter Fahrzeuge insgesamt demnach stark abhängig von der Art des Einsatzes automatisierter Fahrzeuge sowie von den mit ihnen verbundenen Modellierungsannahmen. Dazu gehören vor allem:

$\begin{array}{ll} & \text { Anteil Ride-Sharing } \\ & \text { Veränderungen beim Value of Time } \\ & \text { Siedlungsstruktur } \\ & \\ & \text { Modellannahmen (z. B. Wartezeiten) }\end{array}$

Zudem untersuchen derzeit existierende Modellierungsstudien mehrheitlich den umfangreichen Einsatz weit entwickelter automatisierter Fahrzeuge in ferner Zukunft. Mögliche Effekte in naher Zukunft, wie der etwaige Einsatz von automatisierten Fahrzeugen allein unter bestimmten Bedingungen (ODD, Kap. 4.1), z. B. in bestimmten räumlichen Kontexten (Beiker 2018, S. 125; Shladover 2018, S. 8), waren bisher selten Gegenstand wissenschaftlicher Untersuchungen. Diese sind für die Stadt- und Verkehrsplanung aufgrund des möglichen, relativ zeitnahen Handlungsbedarfs jedoch von weit wichtigerer Bedeutung. Hinzu kommt, dass sich räumliche bzw. generelle Auswirkungen neuer Transporttechnologien, wie auch jene automatisierter Fahrzeuge, bereits viel früher durch Entscheidungen, z. B. den Verzicht des Baus neuer Straßenbahnlinien in Milton Keynes, Großbritannien, oder in Nashville, USA (Smith 2015, The Economist 2018), die in Anbetracht automatisierter Fahrzeuge im Vorfeld getroffen werden, zeigen.

\subsubsection{FOLGEN FÜR STÄDTISCHE BUDGETS}

Neben der im Projekt durchgeführten Untersuchung zu Effekten automatisierter Fahrzeuge auf die Verkehrsnachfrage und die Siedlungsstruktur bzw. Flächennutzung wurden darüber hinaus auch die fiskalischen Effekte automatisierter Fahrzeuge, also deren Wirkungen auf öffentliche Budgets, am Beispiel Österreichs bzw. Wiens untersucht (Mitteregger et al. 2019).

Hierbei wurde davon ausgegangen, dass durch neue Phänomene im Mobilitätssystem - wie insbesondere der Automatisierung und Vernetzung, aber auch der Elektrifizierung ${ }^{1}$ von Fahrzeugen (im Individualverkehr) - primäre Effekte wie mögliche Veränderungen von Fahrzeugbesitz, Sharing, Parkraumbedarf, Verkehrseffizienz sowie Bedarf an Infrastrukturen tragend werden, die letztlich finanzielle Auswirkungen auf öffentliche Budgets (sekundäre Effekte) haben. Zur Untersuchung der fiskalischen Wirkungen der Automatisierung und Vernetzung des Verkehrs wurden auf Basis von Studien in der Literatur die primären Effekte aufgearbeitet sowie die resultierenden sekundären Effekte für Österreich abgeleitet und schließlich der Stellenwert der betroffenen Einnahmenund Ausgabenkategorien in den Budgets der Länder und Gemeinden in Österreich mit speziellem Fokus auf Wien dargestellt. Die budgetären Effekte eines zeitgleichen Wandels im ÖV wurden dabei nicht behandelt.

\section{ÜBERBLICK ÜBER MÖGLICHE FISKALISCHE EFFEKTE²}

Abbildung 4.3.4 gibt einen Überblick über die möglichen sekundären, fiskalischen Effekte durch die Automatisierung, Vernetzung und Elektrifizierung von Fahrzeugen, die sich aus den oben beschriebenen primären Effekten ergeben. Im Bereich der Infrastruktur könnten für die öffentliche Hand deutliche Ausgaben für die Einrichtung bzw. Adaptierung von Verkehrsinfrastruktur aufgrund der Automatisierung sowie für die Errichtung von Ladeinfrastruktur aufgrund der Elektrifizierung anfallen. Auch die Errichtung neuer bzw. die Optimierung bestehender Dateninfrastruktur, d. h. digitaler 


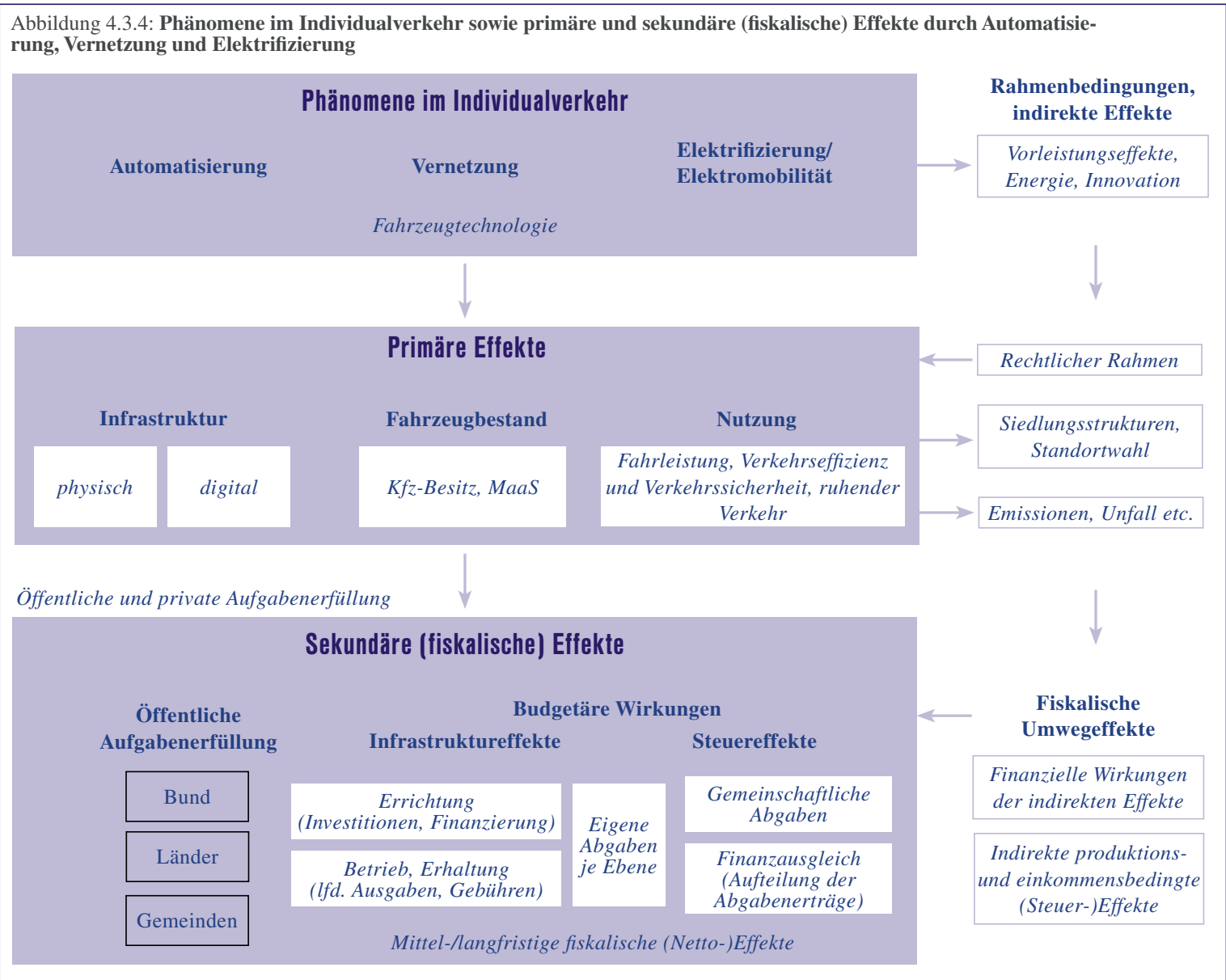

Quelle: AVENUE2

Verkehrsinfrastruktur aufgrund der Vernetzung (und Automatisierung), würde Ausgaben für die öffentliche Hand bedeuten. Der mögliche Rückgang im Fahrzeugbestand durch die Automatisierung könnte zu einer Verminderung bei Einnahmen durch die Normverbrauchsabgabe, die motorbezogene Versicherungssteuer sowie die Kraftfahrzeugsteuer führen. Der mit dem geringeren Fahrzeugbestand einhergehende Parkraumbedarf könnte darüber hinaus auch eine Reduktion der Einnahmen aus der Parkraumbewirtschaftung (Parkometerabgabe bzw. Parkgebühren) zur Folge haben. Zudem könnte der mit der erhöhten Verkehrseffizienz (durch Automatisierung und Vernetzung) verbundene geringere Treibstoffverbrauch einen möglichen Rückgang der Mineralölsteuer mit sich bringen, wobei dies möglicherweise auch durch die erhöhte Nutzung der Fahrzeuge konterkariert werden könnte (Barnes \& Turkel 2017, S. 21). In jedem Fall würde jedoch bereits die Elektrifizierung von Fahrzeugen, d. h. elektrisch angetriebene Fahrzeuge, zusätzlich zu einer Verringerung von Einnahmen aus der Mineralölsteuer führen, wie dies beispielsweise schon heute in Norwegen der Fall ist (POLIS 2018, S. 7). Schließlich könnte das explizite Einhalten von Verkehrsregeln aufgrund der Automatisierung der Fahrzeuge, die ebenso zum Erreichen eines effizienteren Verkehrsflusses von Relevanz ist, zu geringeren Einnahmen aus Verkehrs- strafen, wie etwa für Geschwindigkeitsüberschreitungen oder für Falschparken, führen (Leimenstoll 2017). Insgesamt wird somit deutlich, dass durch automatisierte, vernetzte und elektrisch angetriebene Fahrzeuge vor allem die verkehrsbezogenen Abgaben (d. h. Einnahmen der öffentlichen Hand) sowie Ausgaben für den Bereich Straßenbau, Straßenverkehr sowie Telekommunikationsdienste betroffen sind.

\section{STELLENWERT DER BETROFFENEN EINNAHMEN- UND AUSGABENKATEGORIEN IN DEN BUDGETS DER LÄNDER UND GEMEINDEN IN ÖSTERREICH}

Zwar konnte die genaue Größenordnung der oben beschriebenen sekundären, fiskalischen Effekte - auch mangels näherer quantitativer Bestimmung der primären Effekte - nicht beurteilt werden. Basierend auf der qualitativen Beschreibung der obigen Effekte wurde jedoch eine Betrachtung des aktuellen Stellenwerts der betroffenen Einnahmen- und Ausgabenkategorien der Länder und Gemeinden in Österreich mit speziellem Fokus auf Wien vorgenommen, um so die mögliche Tragweite dieser fiskalischen Effekte aufzuzeigen. Hierzu werden die in Abbildung 4.3.5 in Kursivschrift dargestellten sekun- 
Abbildung 4.3.5: Übersicht über die primären Effekte der Automatisierung, Vernetzung und Elektrifizierung sowie daraus resultierende mögliche fiskalische Effekte

\begin{tabular}{|c|c|c|c|}
\hline \multirow[t]{2}{*}{ PHÄNOMEN } & \multirow[t]{2}{*}{ PRIMÄRER EFFEKT } & \multicolumn{2}{|c|}{ SEKUNDÄRER, FISKALISCHER EFFEKT } \\
\hline & & Einnahmen & Ausgaben \\
\hline Automatisierung & \multirow{3}{*}{ Infrastruktur } & & Verkehrsinfrastruktur \\
\hline Vernetzung & & & $\begin{array}{c}\text { Dateninfrastruktur (digitale } \\
\text { Verkehrsinfrastruktur) }\end{array}$ \\
\hline Elektrifzierung & & Mineralölsteuer & Ladeinfrastruktur, Stromnetz \\
\hline \multirow{3}{*}{ Automatisierung } & \multirow{3}{*}{$\begin{array}{c}\text { Fahrzeugbestand } \\
\text { (Rückgang der Pkws in } \\
\text { Privatbesitz) }\end{array}$} & Normverbrauchsabgabe & \\
\hline & & Motorbezogene Versicherungssteuer & \\
\hline & & Kraftfahrzeugsteuer & \\
\hline $\begin{array}{l}\text { Automatisierung } \\
\text { Automatisierung und } \\
\text { Vernetzung }\end{array}$ & $\begin{array}{c}\text { Nutzung } \\
\text { Parkraumbedarf } \\
\text { Verkehrseffizienz und } \\
\text { Verkehrssicherheit }\end{array}$ & $\begin{array}{c}\text { Parkraumbewirtschaftung } \\
\text { (Parkometerabgabe/-gebühren) } \\
\text { Park-/Verkehrsstrafen } \\
\text { Mineralölsteuer }{ }^{2}\end{array}$ & Parkinfrastruktur \\
\hline
\end{tabular}

dären, fiskalischen Effekte (soweit möglich) mit Daten unterlegt. $^{3}$

\section{EINNAHMEN}

In Österreich werden die oben beschriebenen verkehrsbezogenen Einnahmen (gemeinschaftliche Bundesabgaben), wie beispielsweise die Mineralölsteuer, grundsätzlich zunächst vom Bund erhoben. Die Länder und Gemeinden erhalten davon dann einen Anteil, der nach den Verteilungsregeln im Finanzausgleichgesetz bestimmt wird (BMF 2018, Bröthaler et al. 2017).

Blickt man auf die gemeinschaftlichen Bundesabgaben (Tab. 4.3.1), so wird deutlich, dass sich verkehrsbezogene Abgaben wie Mineralölsteuer, Normverbrauchsabgabe, motorbezogene Versicherungssteuer, Kraftfahrzeugsteuer und Versicherungssteuer durch eine Verlagerung zu automatisiert, vernetzt und/oder elektrisch angetriebenen Fahrzeugen verändern. Eine Betrachtung dieses Effekts ist wesentlich, machten diese Abgaben doch beispielsweise im Jahr 2016 mit insgesamt rund 8,5 Mrd. Euro einen Anteil von 10,7 \% an den gesamten gemeinschaftlichen Bundesabgaben aus. Die Mineralölsteuer sowie die motorbezogene Versicherungssteuer besitzen dabei mit 5,6 \% bzw. 3,0 \% den größten Anteil. Im zeitlichen Verlauf ist der Anteil der verkehrsbezogenen Abgaben an den gemeinschaftlichen Bundesabgaben relativ stabil.

Blickt man auf die Einnahmen der Länder und Gemeinden nach der Anwendung der Verteilungsschlüssel für die gemeinschaftlichen Bundesabgaben (Abb. 4.3.2), zeigt sich, dass die Ertragsanteile an verkehrsbezogenen (gleichwohl nicht zweckgewidmeten) Abgaben im Jahr 2017 bei den Ländern ohne Wien sowie den Gemeinden ohne Wien einen Anteil von 4,0 \% bzw. 3,7 \% der gesamten Einnahmen hatten. Speziell für Wien zeigt sich für Erträge aus verkehrsbezogenen Abgaben von 631 Mio. Euro ein Anteil von 4,3\%. Einnahmen aus der Parkometerabgabe und Parkstrafen, die durch automatisierte, vernetzte und elektrisch angetriebene Fahrzeuge ebenfalls

Tabelle 4.3.1: Aufkommen an gemeinschaftlichen Bundesabgaben 2007 und 2017 in Mio. Euro bzw. in Prozent

\begin{tabular}{|c|c|c|c|c|}
\hline $\begin{array}{l}\text { GEMEINSCHAFTLICHE } \\
\text { BUNDESABGABEN }\end{array}$ & 2007 MIO. € & 2017 MIO.€ & $\%$ P.A & 2017 \%-ANTEIL \\
\hline Mineralölsteuer & 3.689 & 4.436 & 1,9 & 5,6 \\
\hline Normverbrauchsabgabe & 456 & 469 & 0,3 & 0,6 \\
\hline Motorbezogene Versicherungssteuer & 1.410 & 2.389 & 5,4 & 3 \\
\hline Kraftfahrzeugsteuer & 1.115 & 38 & $-10,4$ & 0 \\
\hline Versicherungssteuer & 993 & 1.128 & 1,3 & 1,4 \\
\hline Verkehrsbezogene Abgaben gesamt & 6.663 & 8.461 & 2,4 & 10,7 \\
\hline Ertragssteuern & 30.516 & 39.269 & 2,6 & 49,5 \\
\hline Umsatzsteuer & 19.212 & 25.519 & 2,9 & 32,2 \\
\hline Sonstige gemeinschaftliche Abgaben & 3.870 & 6.015 & 4,5 & 7,6 \\
\hline Gesamtaufkommen & 60.261 & 79.264 & 2,8 & 100 \\
\hline
\end{tabular}


Tabelle 4.3.2: Einnahmen der Länder und Gemeinden aus Abgaben und sonstigen Einnahmen 2017 in Mio. Euro bzw. Anteil in Prozent der gesamten Einnahmen

\begin{tabular}{|c|c|c|c|c|c|c|}
\hline \multirow[b]{2}{*}{ EINNAHMEN 2017} & \multicolumn{3}{|c|}{ IN MIO. EURO } & \multicolumn{3}{|c|}{ IN PROZENT } \\
\hline & $\begin{array}{c}\text { Länder ohne } \\
\text { Wien }\end{array}$ & $\begin{array}{l}\text { Gemeinden } \\
\text { ohne Wien }\end{array}$ & Wien & $\begin{array}{l}\text { Länder ohne } \\
\text { Wien }\end{array}$ & $\begin{array}{l}\text { Gemeinden } \\
\text { ohne Wien }\end{array}$ & Wien \\
\hline Verkehrsbezogene Abgaben gesamt & 1.369 & 741 & 631 & 4,0 & 3,7 & 4,3 \\
\hline Ertragssteuern & 6.344 & 3.432 & 2.922 & 18,4 & 17,1 & 19,9 \\
\hline Umsatzsteuer & 4.232 & 2.308 & 1.713 & 12,3 & 11,5 & 11,7 \\
\hline Sonstige gemeinschaftliche Abgaben & 1.298 & 1.057 & 793 & 3,8 & 5,3 & 5,4 \\
\hline Ertragsanteile an gem. Bundesabgaben & 13.244 & 7.537 & 6.059 & 38,4 & 37,7 & 41,2 \\
\hline Parkometerabgabe inkl. -gebühren & & 70 & 115 & & 0,3 & 0,8 \\
\hline Sonstige eigene Abgaben & 679 & 3.448 & 1.294 & 2,0 & 17,2 & 8,8 \\
\hline Abgabeeinnahmen gesamt & 13.923 & 11.055 & 7.468 & 40,4 & 55,2 & 50,8 \\
\hline $\begin{array}{l}\text { Park-/Verkehrsstrafen } \\
\text { (mit Zweckbindung) }\end{array}$ & 50 & 70 & 82 & 0,1 & 0,4 & 0,6 \\
\hline Sonstige laufende Einnahmen & 15.789 & 5.362 & 4.261 & 45,8 & 26,8 & 29,0 \\
\hline Vermögenseinnahmen & 4.705 & 3.526 & 2.882 & 13,7 & 17,6 & 19,6 \\
\hline Gesamte Einnahmen & 34.466 & 20.013 & 14.693 & 100,0 & 100,0 & 100,0 \\
\hline
\end{tabular}

Quelle: AVENUE21 nach Gebarungsstatistik, Statistik Austria (2019a)

vor Veränderungen stehen, machten im Jahr 2017 mit 115 Mio. Euro bzw. 82 Mio. Euro zusammen rund 1,4 \% der gesamten Einnahmen Wiens aus.

\section{AUSGABEN}

Ein Blick auf die Ausgaben der Länder ohne Wien sowie der Gemeinden ohne Wien (Tab. 4.3.2) zeigt, dass die - durch automatisierte, vernetzte und elektrisch angetriebene Fahrzeuge möglicherweise veränderten - Ausgaben für Straßenbau und Straßenverkehr im Jahr 2017 einen Anteil von 3,7 \% (Länder ohne Wien) bzw. 7,8 \% (Gemeinden ohne Wien) an deren Gesamtausgaben ausmachte. Für Wien zeigt sich hier mit 261 Mio. Euro ein Anteil von $1,8 \%$ an den Gesamtausgaben. Im zeitlichen Verlauf zeigt sich, dass der Anteil der Ausgaben für Straßen in Wien in den letzten zehn Jahren auf ähnlichem Niveau verblieben ist, während er bei den Ländern ohne Wien und bei den Gemeinden leicht gesunken ist.

Insgesamt machen Einnahmen- und Ausgabenkategorien, die von der Einführung automatisierter, vernetzter und elektrisch angetriebener Fahrzeuge betroffen sind, somit einen nicht unwesentlichen Anteil an den Gesamteinnah- men und Gesamtausgaben der Länder und Gemeinden Österreichs sowie im Speziellen auch der Stadt Wien aus.

1 Die Elektrifizierung von Fahrzeugen (Umstieg auf Elektroantrieb) wurde miteinbezogen, obwohl diese per se nicht an die Automatisierung gebunden ist. Die Gleichzeitigkeit der beiden Phänomene wird jedoch in der Literatur häufig betont (z. B. Bormann et al. 2018)

2 In diesem Abschnitt werden in gekürzter Form die Erkenntnisse aus „Shared, Automated, Electric: the Fiscal Effects of the Holy Trinity“ (Mitteregger et al. 2019) dargestellt. Die Erweiterung der Analyse möglicher Effekte von avV hinsichtlich gemeindefiskalischer Wirkungen wäre ohne die Co-Autorenschaft von Johann Bröthaler (Forschungsbereich Finanzwissenschaft und Infrastrukturpolitik der TU Wien - IFIP) nicht möglich gewesen.

3 Hierbei gilt es zu berücksichtigen, dass die Eingrenzung auf der Einnahmenseite relativ genau möglich ist, jedoch keine Zweckbindung der (Abgaben-)Einnahmen vorliegt. Auf der Ausgabenseite ist demgegenüber aufgrund der haushaltsrechtlichen Kategorisierung sowie der unterschiedlichen Verbuchungen und institutionellen Rahmenbedingungen (Aufgabenkompetenzen) zum Teil nur eine gröbere Eingrenzung möglich (insbesondere betreffend Digitalisierung und Stromversorgung/Ladeinfrastruktur). Darüber hinaus beschränkt sich die empirische Darstellung auf die Haushalte der Länder und Gemeinden als Gebietskörperschaften (ohne ausgegliederte bzw. außerbudgetäre Einheiten).

Tabelle 4.3.3: Ausgaben der Länder und Gemeinden für Straßen und ÖV 2017 in Mio. Euro

\begin{tabular}{|l|c|c|c|c|c|c|}
\hline \multirow{2}{*}{ AUSGABEN 2017 } & \multicolumn{3}{|c|}{ IN MIO. EURO } & \multicolumn{3}{c|}{ IN PROZENT } \\
\cline { 1 - 8 } & $\begin{array}{c}\text { Länder ohne } \\
\text { Wien }\end{array}$ & $\begin{array}{c}\text { Gemeinden } \\
\text { ohne Wien }\end{array}$ & Wien & $\begin{array}{c}\text { Länder ohne } \\
\text { Wien }\end{array}$ & $\begin{array}{c}\text { Gemeinden } \\
\text { ohne Wien }\end{array}$ & Wien \\
\hline Straßen & 1.283 & 1.783 & 261 & 3,7 & 7,8 & 1,8 \\
\hline ÖV & 580 & 161 & 774 & 1,7 & 0,7 & 5,3 \\
\hline Sonstige Ausgaben & 32.639 & 21.007 & 13.658 & 94,6 & 91,5 & 93,0 \\
\hline Gesamtausgaben & 34.502 & 22.952 & 14.693 & 100,0 & 100,0 & 100,0 \\
\hline
\end{tabular}


Abbildung 4.3.6: Wirkungen automatisierter Fahrzeuge im SAE-Level 5

\begin{tabular}{|c|c|}
\hline \multicolumn{2}{|c|}{ FAHRZEIT } \\
\hline \multicolumn{2}{|c|}{ Verringerung Zeitwahrnehmung } \\
\hline r & $\begin{array}{l}\text { - höhere Produktivität (Durchführung anderer } \\
\text { Aktivitäten) } \\
\text { - höherer Fahrkomfort (optimierte Längs- und } \\
\text { Querbeschleunigung, Vermeidung fahrbedingten } \\
\text { Stresses) }\end{array}$ \\
\hline$\square$ & $\begin{array}{l}\text { - Passagiere in avF anfälliger für Reise- bzw. } \\
\text { Bewegungskrankheit } \\
\text { - ungewohnte bzw. mangelnde Erfahrung im } \\
\text { Umgang mit avF } \\
\text { - } \quad \text { geringe Abstände zwischen Fahrzeugen }\end{array}$ \\
\hline \multicolumn{2}{|c|}{ Verringerung der Fahrzeit } \\
\hline 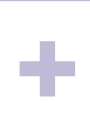 & $\begin{array}{l}\text { - Wegfall von Parkplatzsuchverkehr } \\
\text { - Entfall von Parkvorgängen und Weg zum } \\
\text { Parkplatz/Zielort }\end{array}$ \\
\hline \multicolumn{2}{|c|}{ Verlässlichkeit der Fahrzeit } \\
\hline L & $\begin{array}{l}\text { - nahezu konstante Geschwindigkeiten } \\
\text { - } \quad \text { verlässliche und vorhersagbare Routen }\end{array}$ \\
\hline
\end{tabular}

\begin{tabular}{|c|c|}
\hline \multicolumn{2}{|c|}{ KOSTEN } \\
\hline \multicolumn{2}{|c|}{ Verringerung der Betriebskosten } \\
\hline r & $\begin{array}{ll}\text { - } & \text { treibstoffeffizientere und leichtere Fahrzeuge } \\
\text { - } & \text { treibstoffsparende Fahrzeugstile } \\
\text { - } & \text { geringere Kosten für Kfz-Versicherung } \\
\text { - } & \text { Einsparung von Personalkosten im ÖV } \\
\text { - } & \begin{array}{l}\text { Rückgang der Verschleißkosten (z. B. für Reifen) } \\
\text { im ÖV }\end{array}\end{array}$ \\
\hline \multicolumn{2}{|c|}{ Höhere Anschaffungskosten } \\
\hline - & - teure verbaute Sensorik und Softwarekomponenten \\
\hline 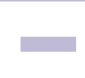 & $\begin{array}{l}\text { - Entfall von fahrerbezogener Ausstattung innerhalb } \\
\text { des Fahrzeugs (Lenkrad, Brems- und Gaspedal) }\end{array}$ \\
\hline \multicolumn{2}{|c|}{ Kosten pro Personenkilometer (inkl. Abschreibung) } \\
\hline \multicolumn{2}{|c|}{$-16 \%$ bis $+4 \%$} \\
\hline \multicolumn{2}{|c|}{ Sinkende Parkkosten } \\
\hline - & $\begin{array}{l}\text { - Parken in günstigeren Gebieten bzw. Gebieten } \\
\text { ohne Parkgebühren }\end{array}$ \\
\hline
\end{tabular}

\begin{tabular}{|c|c|}
\hline \multicolumn{2}{|c|}{ SICHERHEIT } \\
\hline \multicolumn{2}{|c|}{ Erhöhung Verkehrssicherheit } \\
\hline 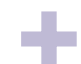 & - Reduktion fahrerrelevanter Unfallursachen \\
\hline 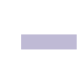 & $\begin{array}{l}\text { - unklar während der Übergangszeit und im } \\
\text { Mischverkehr }\end{array}$ \\
\hline \multicolumn{2}{|c|}{ Unfälle } \\
\hline \multicolumn{2}{|c|}{$-70 \%$ bis $-95 \%$} \\
\hline \multicolumn{2}{|c|}{ Geringe Cybersicherheit } \\
\hline 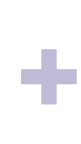 & $\begin{array}{l}\text { - } \text { Fehler in der Software } \\
\text { - Cyberangriffe } \\
\text { - } \quad \text { Überwachung/Probleme beim Datenschutz }\end{array}$ \\
\hline
\end{tabular}

Quelle: BMVIT (2016b)

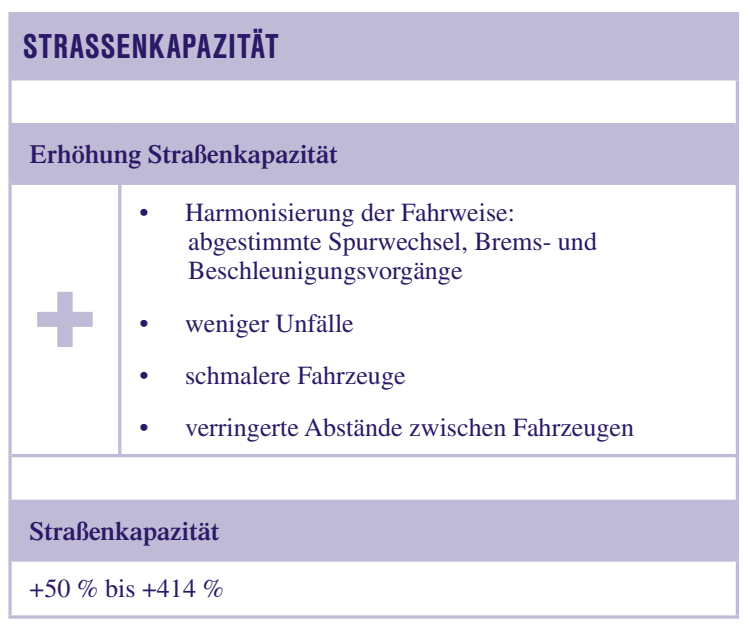

Quelle: Van den Berg \& Verhoef (2016), Bösch (2016)

\section{NEUE NUTZERGRUPPEN}

\section{Erschließung neuer Nutzergruppen}

\begin{tabular}{|c|c|}
\hline 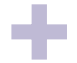 & $\begin{array}{l}\text { - geringere Anforderungen an FahrerInnen/ } \\
\text { PassagierInnen (kein Führerschein nötig) }\end{array}$ \\
\hline \multicolumn{2}{|c|}{ Mobilitätsbeeinträchtigte Personen in Österreich } \\
\hline $10 \%$ & \\
\hline
\end{tabular}




\begin{tabular}{|c|c|}
\hline \multicolumn{2}{|c|}{ ENERGIE UND UMWELT } \\
\hline \multicolumn{2}{|c|}{ Energieeffizienz (Treibstoffeinsparungen) } \\
\hline \multicolumn{2}{|c|}{$-11 \%$ bis $-47 \%$} \\
\hline \multicolumn{2}{|c|}{ Emissionen } \\
\hline $\mathrm{CO}$ & $-32 \%$ bis $-61 \%$ \\
\hline $\mathrm{CO}_{2}$ & $-61 \%$ bis $+105 \%$ \\
\hline $\mathrm{NO}_{x}$ & $-2 \%$ bis $-18 \%$ \\
\hline \multicolumn{2}{|c|}{$\begin{array}{l}\text { Hinsichtlich der Wirkungen automatisierter Fahrzeuge auf } \\
\text { Energie und Umwelt existieren vereinzelt Studien. Allerdings } \\
\text { besteht auch hier eine große Spannweite zwischen den Effekten } \\
\text { Weiterer Forschungsbedarf besteht vor allem auch hinsichtlich } \\
\text { der Berücksichtigung der Effekte im Bereich Verkehr und } \\
\text { Mobilität. }\end{array}$} \\
\hline
\end{tabular}

Quelle: Fagnant, Kockelman \& Bansal (2015), Milakis et al. (2017), Wadud et al. (2016)

\begin{tabular}{|c|c|}
\hline \multicolumn{2}{|c|}{ WIRTSCHAFT UND ÖFFENTLICHE FINANZEN } \\
\hline Nutzen pro Fahrzeug & $€ 2.960$ bis $€ 3.900$ \\
\hline Wertschöpfung & $+30 \%$ \\
\hline $\begin{array}{l}\text { Wegfall von Steuern/ } \\
\text { Einnahmen }\end{array}$ & $\begin{array}{l}\text { aktueller Stellenwert in } \\
\text { Österreich }\end{array}$ \\
\hline Normverbrauchsabgabe & $€ 2.960$ bis $€ 3.900$ \\
\hline $\begin{array}{l}\text { Motorbezogene } \\
\text { Versicherungssteuer }\end{array}$ & $+30 \%$ \\
\hline Parkometerabgabe & $€ 2.960$ bis $€ 3.900$ \\
\hline Park-/Verkehrsstrafen & $+30 \%$ \\
\hline $\begin{array}{l}\text { In diesem Bereich existiere } \\
\text { zeigen Studien den derzeiti } \\
\text { Wegfalls von Einnahmeque } \\
\text { hoch dieser tatsächlich ist, } \\
\text { und von obigen Wirkungen }\end{array}$ & $\begin{array}{l}\text { sher kaum Studien. Allenfalls } \\
\text { Stellenwert des möglichen } \\
\text { der öffentlichen Hand auf; wie } \\
\text { edoch noch weitgehend ungeklärt } \\
\text { rängig. }\end{array}$ \\
\hline
\end{tabular}

Quelle: Clements \& Kockelman (2017), 
4.4

\section{AUTOMATED DRIVABILITY: EIN DIFFERENZIERTES BILD DES RÄUMLICHEN EINSATZES VON AUTOMATISIERTEN UND VERNETZTEN FAHRZEUGEN}

Einblick in die Dissertation von Aggelos Soteropoulos

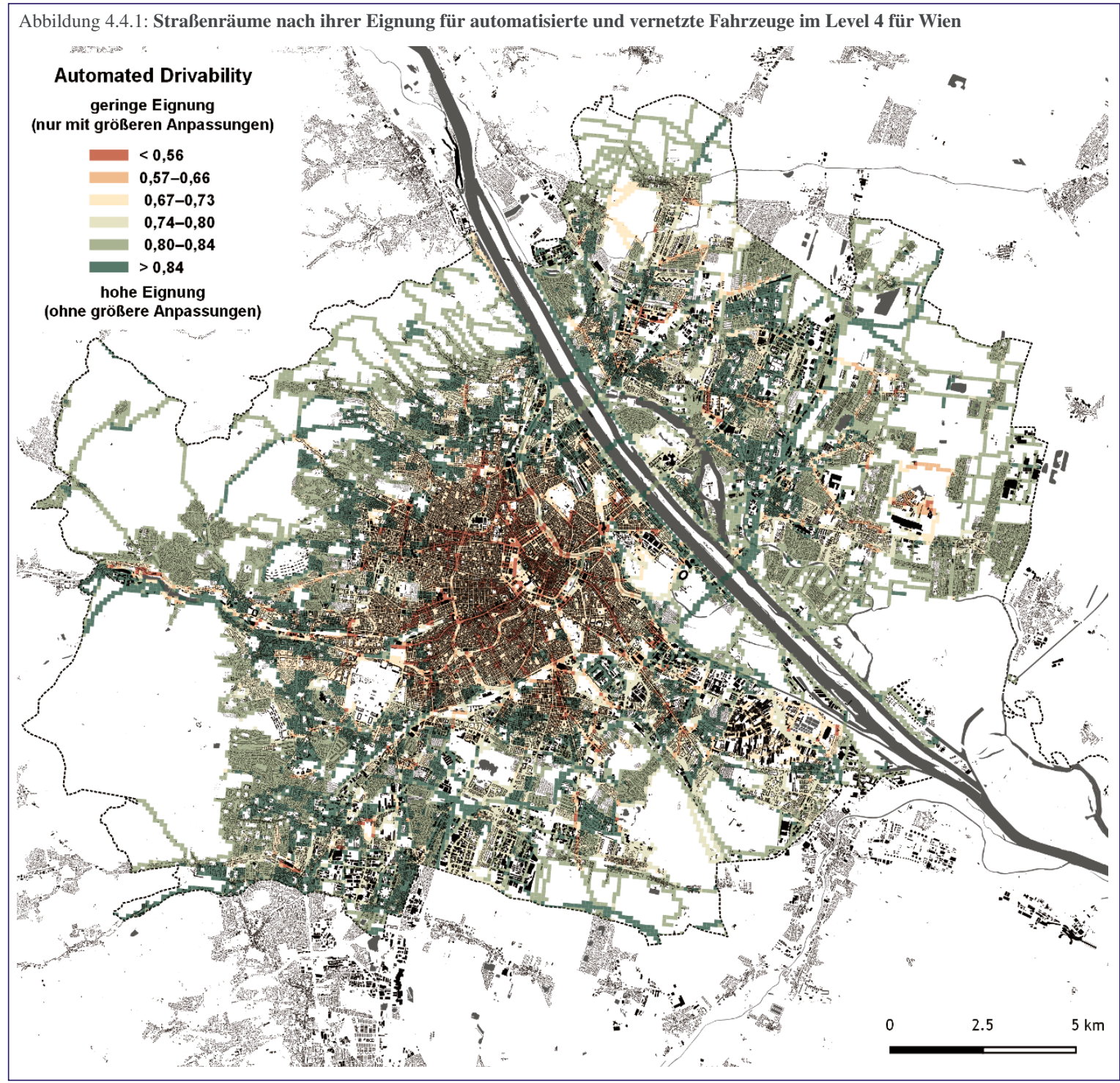

Quelle: AVENUE2

Wie in Kapitel 4.3 dargelegt, wurden in Simulationen und Prognosen zum Einsatz von avF die qualitativen Unterschiede von Straßenräumen nur bedingt berücksichtigt. So geht man zwar heute z. B. davon aus, dass Autobahnen oder Sonderareale „leichtere“ Automatisierungsaufgaben darstellen und folglich automatisierte Fahrsysteme (SAE International 2018 definiert hier noch keine Vernetzung) dort früher zum Einsatz kommen (s. auch Kap. 4.1). Jenseits dieser groben Gegenüberstellung jedoch fehlt ein differenzierter Blick mit straßenräumlichen Eigenschaften und Umfeldbedingungen innerhalb beider Kategorien. Der Begriff der Operational Design Domain (ODD) wurde in den bekannten SAE-Levels mit der Überarbeitung im Jahr 2016 einge- 


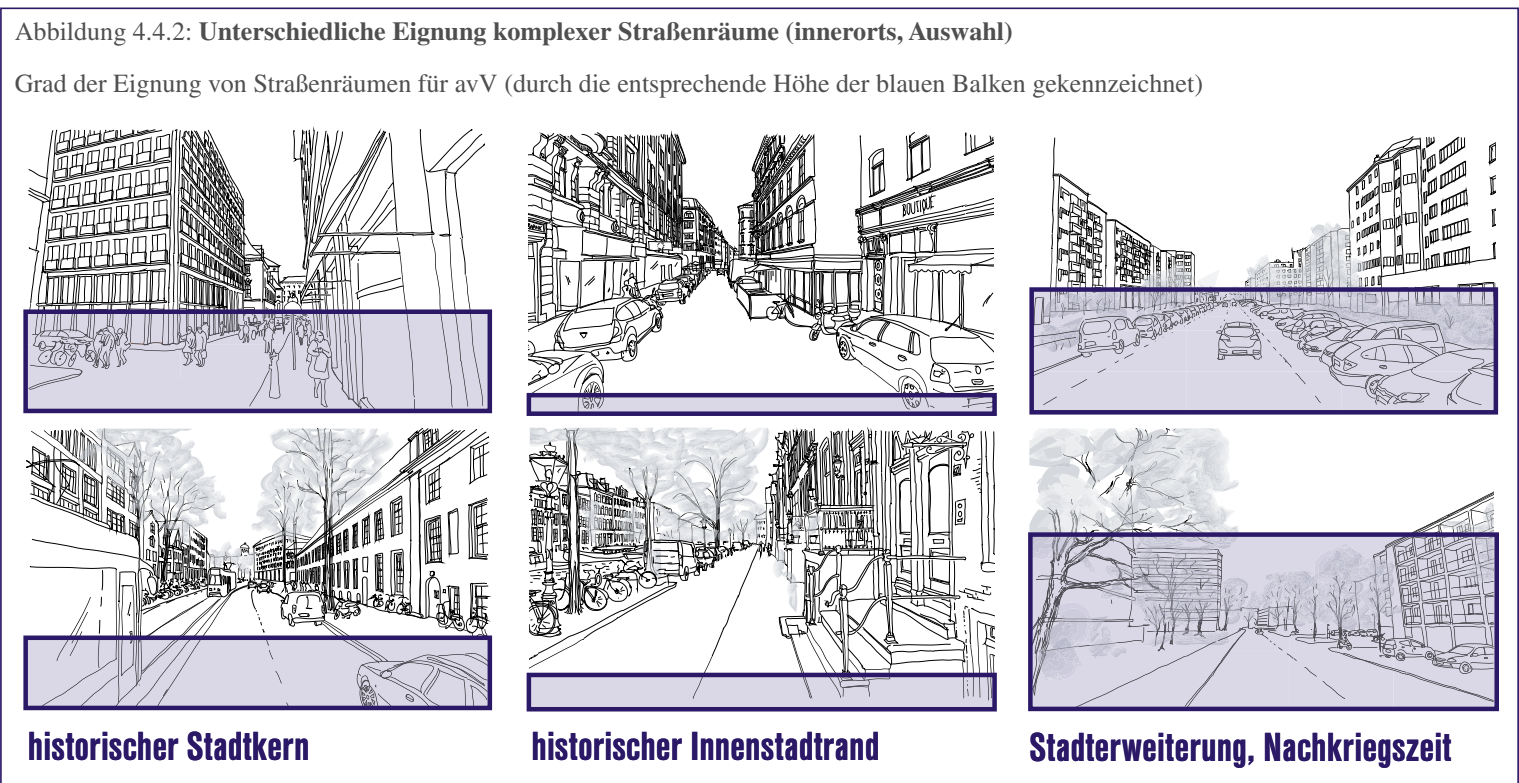

Quelle: AVENUE21

führt, um die Bandbreite an unterschiedlich komplexen Umfeldern für Fahrzeugautomatisierung darzustellen (SAE International 2018, S. 14). Ein automatisiertes Fahrsystem kann zum Beispiel nur bei geringer Geschwindigkeit, bei gutem Wetter und nur am Tag funktionieren (Fraade-Blanar et al. 2018, S. 13). Diese Einschränkungen entsprächen der ODD, auf die das System ausgelegt wurde.

Im Forschungsprojekt AVENUE21 wurde ein erster Schritt für die Entwicklung eines Index der Automated Drivability entwickelt, um die Anforderungen an automatisierte Fahrsysteme differenzierter zu betrachten, die direkt von der Komplexität der ODD abhängen (s. Abb. 4.4.3). Der Ausgangspunkt des Index liegt darin begründet, dass bestimmte straßenräumliche Kontexte die Anforderungen an automatisierte Fahrsysteme erhöhen (Metz 2018, S. 3). Dies ergibt sich insbesondere aus deren Funktionsweise: Automatisierte Fahrsysteme müssen das Umfeld mit unterschiedlichen Sensoren erfassen, dieses anhand derer abbilden (Wahrnehmung und Kognition), anschließend die entsprechenden Fahrentscheidungen (Planung und Kontrolle) treffen und diese den PassagierInnen und den anderen
Verkehrsteilnehmenden kommunizieren (Mensch-Maschine-Interaktion; Ritz 2018, S. 41). Unterschiedliche Einsatzumgebungen und die damit verbundenen Rahmenbedingungen können diese Prozesse erschweren, beispielsweise wenn eine Vielzahl unterschiedlicher VerkehrsteilnehmerInnen erkannt werden muss und auch deren zukünftige Bewegungen vorhergesehen bzw. antizipiert werden müssen (Shladover 2018, S. 31).

\section{EUROPÄISCHE STÄDTE: VIELFALT VON STRASSENRÄUMLICHEN KONTEXTEN}

Gerade in europäischen Städten besteht eine Vielfalt von Straßenräumen, die sich hinsichtlich ihrer baulichen und infrastrukturellen Ausgestaltung, der umgebenden Architektur sowie der Differenziertheit der Verkehrsteilnehmenden deutlich unterscheiden und damit auch sehr unterschiedliche Anforderungen für automatisierte Fahrsysteme stellen (s. Abb. 4.4.2). Straßenräume weisen dabei unterschiedliche Funktionen (Verbindungsoder Erschließungsfunktion) sowie Verkehrsbelastungen bzw. Verkehrsstärken auf (FGSV 2006, S. 8; Marshall

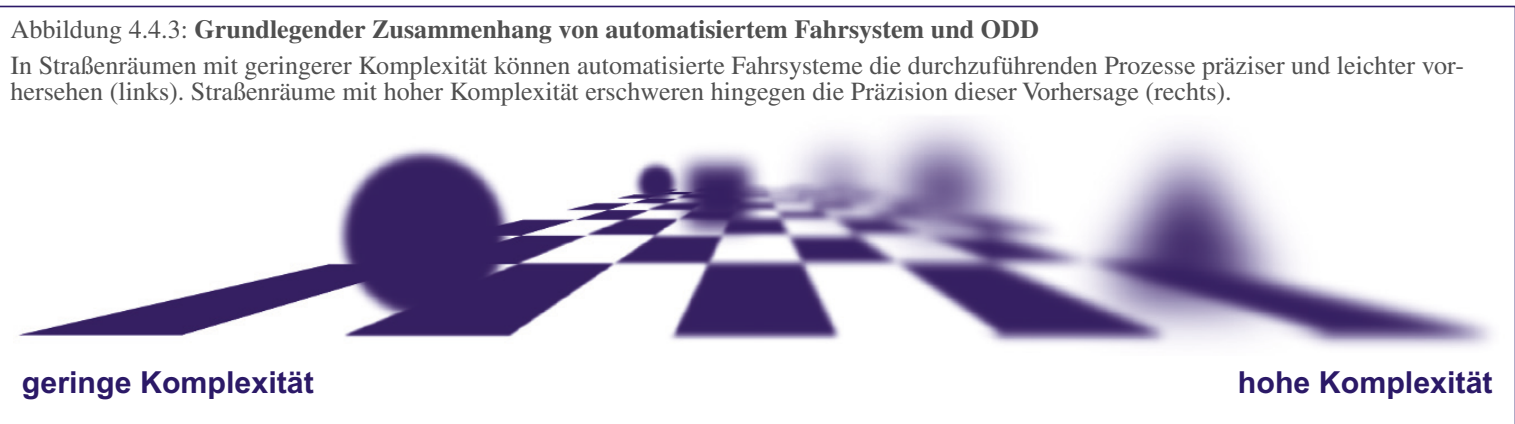


2005, S. 50). Auch die Knotenpunkte als Verbindungspunkte zwischen den Straßen sind vielfältig (Rechtsvor-links-Regelung, vorfahrtregelnde Verkehrszeichen, Lichtsignalanlagen, Kreisverkehre etc.) und abhängig von den Eigenschaften der zu verknüpfenden Straßen (FGSV 2006, S. 54).

\section{FAKTOREN FÜR DIE EIGNUNG VON STRASSENRÄUMEN FÜR DEN EINSATZ VON AUTOMATISIERTEN UND VERNETZTEN FAHRZEUGEN: AUTOMATED DRIVABILITY}

Ausgehend davon wurden Faktoren erarbeitet, welche die Eignung von Straßen- bzw. Verkehrsräumen für den Einsatz von avF, die Automated Drivability, abbilden und damit auch den räumlich differenzierten Einsatz von avF bestimmen. Diese Faktoren umfassen (s. Abb. 4.4.4):

1 die Anzahl der Objekte im Straßenraum,

2 die Verschiedenheit der Objekte im Straßenraum,

3 die Stabilität der ODD,

4 den erlaubten Geschwindigkeitsbereich und

5 den Zustand der Infrastruktur.

\section{ABSCHÄTZUNG DER AUTOMATED DRIVABILITY: FALLBEISPIEL WIEN}

Am Beispiel der Stadt Wien wurden, vor dem Hintergrund der erarbeiteten Faktoren und in Abhängigkeit von der Datenverfügbarkeit, beispielhafte Kriterien abgeleitet und kombiniert, welche die erarbeiteten Faktoren und damit die Eignung von Straßenräumen für den funktionierenden Einsatz automatisierter Fahrsysteme so gut wie möglich abbilden (s. Abb. 4.4.5). Die verwendeten Kriterien sind ein Versuch, diese Heterogenität unterschiedlicher Straßenräume in ihrer Verortung in der Stadt abzubilden. Abbildung 4.4.1 zeigt die Abschätzung der Automated Drivability am Beispiel der Stadt Wien. Hierbei wird erkennbar, dass Städte hinsichtlich der Einsatzmöglichkeit von avF keineswegs homogene Räume darstellen. Vielmehr zeigt sich anhand der abgeleiteten Kriterien, dass ein räumlich selektiver Einsatz von avF aufgrund der unterschiedlichen Eignung der Straßenräume in Städten wahrscheinlich sein wird und die Vor- und Nachteile des Einsatzes von avF in der ausgedehnten Übergangszeit ungleichmäßig verteilt sein werden.

Eine Folge dieser Heterogenität ist die Verschiebung der Lagegunst durch die Erreichbarkeit mit avF im
Abbildung 4.4.4: Kurzbeschreibung zu den erarbeiteten Faktoren

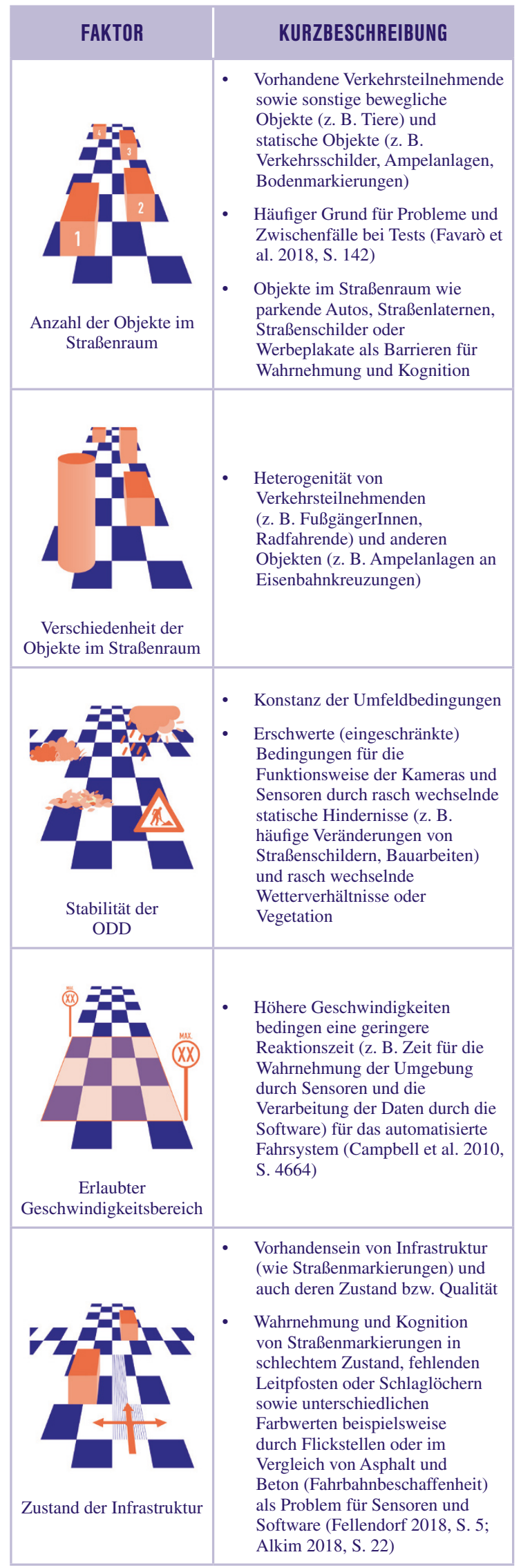

Quelle: AVENUE21 


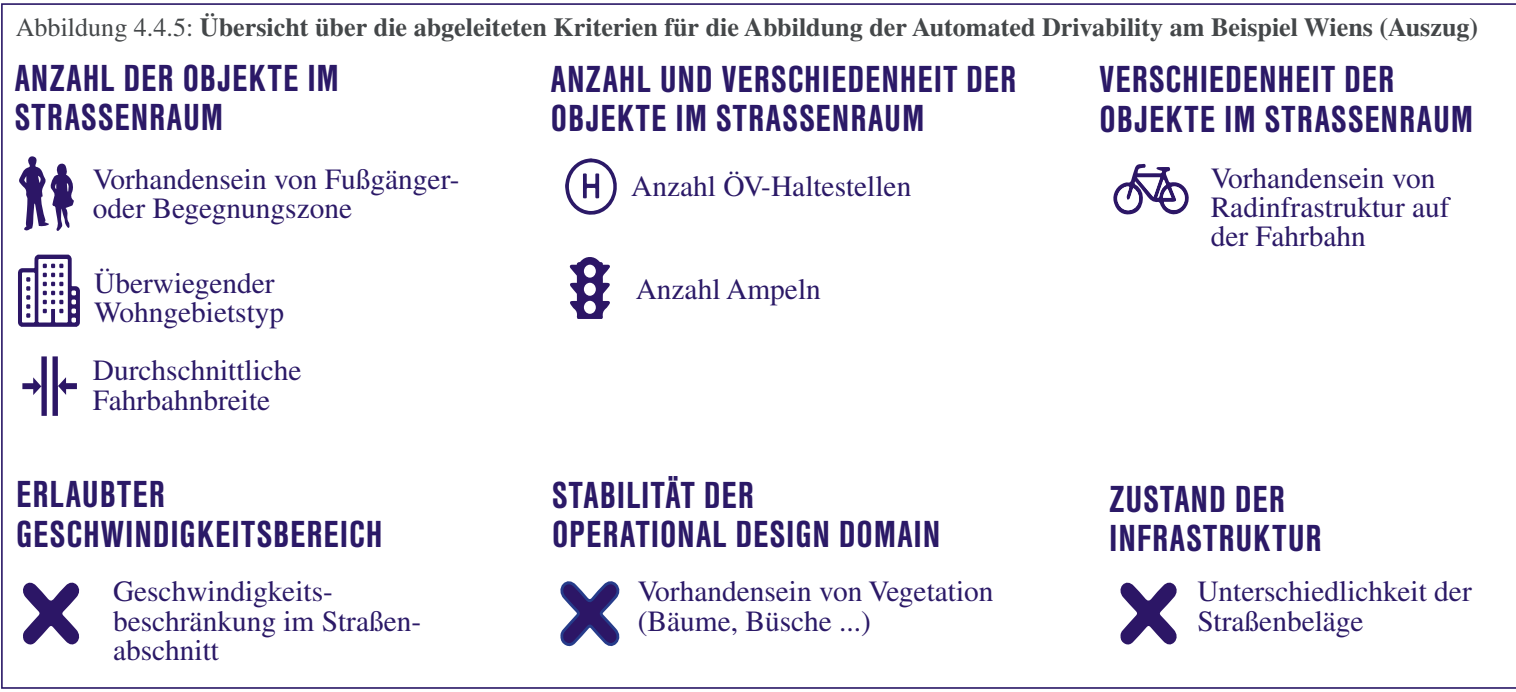

Quelle: AVENUE21

Personen- und Güterverkehr. Erkennbar ist, dass eher autoaffinere periphere Stadtbereiche (die in Abb. 4.4.1 grün gekennzeichnet sind) tendenziell einen Vorteil gegenüber älteren, meist komplexeren Stadtquartieren im Zentrum haben. Während Erstere ohne (größere) Anpassungen und vermutlich relativ bald erschlossen werden könnten, wäre bei Letzteren ein Einsatz von avF nur denkbar, wenn größere Anpassungen im Straßenraum vorgenommen (gebaute Infrastruktur), die Geschwindigkeit grundsätzlich reduziert und eine Ertüchtigung durch digitale Infrastruktur erfolgen würden (rot markiert) - hierzu gibt es jedoch noch keinerlei Standards. Darüber hinaus wird deutlich, dass für europäische Städte, die häufig auf FußgängerInnen ausgerichtete historische Siedlungskerne mit engen Gassen haben, den motorisierten und vernetzten Verkehr nicht aufnehmen können. Transportation Network Companies wie Uber oder Lyft reagieren mittlerweile darauf, indem sie ihre Dienste dort auf Zweirad-Fahrzeuge verlagern.

Mit der Abschätzung des Potenzials für Wien wird ein erster Überblick zur Eignung von Verkehrsräumen für den Einsatz von avF gegeben. Mit weiteren Daten kann die Analyse differenziert werden. Nichtsdestotrotz stellt die Darstellung der Automated Drivability Wiens (Abb. 4.4.1) vor dem Hintergrund des erarbeiteten Index einen Überblick von Flächen dar, die ohne (größere) Anpassungen durch avF erschlossen werden können und die sich keineswegs gleichmäßig über die Stadt verteilen werden. Zusätzlich könnte mittels einer Sozialraumanalyse festgestellt werden, welche sozialen Gruppen eher in den grün oder rot gekennzeichneten Straßen leben und welche Mobilitätsstile dort dominieren. Dieses Wissen ist von zentraler Bedeutung für Politik und Planung, um Maßnahmen differenziert entwickeln zu können. 
4.5

\section{TRANSITION MANAGEMENT IN INTERNATIONALEN VORREITERREGIONEN}

Im Kontext der Auswirkungen der avM auf Stadt(regionen) können sogenannte Vorreiterregionen (,Pioneering Regions") - Städte oder Stadtregionen, die sich in der Forschung, Planung und Entwicklung sowie in der Erprobung und Demonstration von avF und avM besonders engagieren und die Umsetzung von avM angesichts regionaler Herausforderungen forcieren - beschrieben werden.

Gegenstand der Betrachtung in diesem Kapitel sind die Herausforderungen, die Städte und Stadtregionen mit dem Einsatz von avF adressieren wollen, und die Transition-Initiativen, durch die Städte und Stadtregionen zu Co-Produzenten der Entwicklung von avF werden.

\section{AUSWAHL DER VORREITERREGIONEN}

Im Zuge der Recherche wurden etwa zwanzig Stadtregionen ausgewählt, untersucht und miteinander verglichen. Nach klärenden Diskussionen mit ExpertInnen wurden fünf von ihnen als Vorreiterregionen herausgefiltert: Sie liegen in den USA (San Francisco), in Großbritannien (Stadtregion London), in Schweden (Göteborg) und in Japan (Stadtregion Tokio). Als fünfte Vorreiterregion fiel der Stadtstaat Singapur in die Auswahl (s. Abb. 4.5.1). Das Ziel der Studie war es, eine möglichst große Bandbreite von unterschiedlichen Referenzen heranzuziehen, die relevante Zukunftsvorstellungen und Entwicklungsmöglichkeiten aufzeigt, in denen Transition-Initiativen in Strategien und Programmen sichtbar werden und die für ihre Beiträge zum globalen Diskurs um den avV bereits internationale Anerkennung gefunden haben.

\section{DIE VIER ANALYTISCHEN KATEGORIEN}

Zur Analyse der Vorreiterregionen wurde der theoretische Rahmen der Transition Theory (Rotmans et al. 2001, Kemp \& Loorbach 2003, Geels 2005) und der Multi-Ebenen-Perspektive (Multi Level Perspective - MLP; Geels 2010) herangezogen. Die heuristische Methode der MLP sieht Systemübergänge als relationale Prozesse an, die aus der Interaktion zwischen den Entwicklungen in drei Dimensionen resultieren (Rotmans et al. 2001):

- Die Dimension „Landscape“ beinhaltet lokale oder translokale Aspekte eines Ortes/einer Region, wie beispielsweise die Auswirkungen des demographischen Wandels.

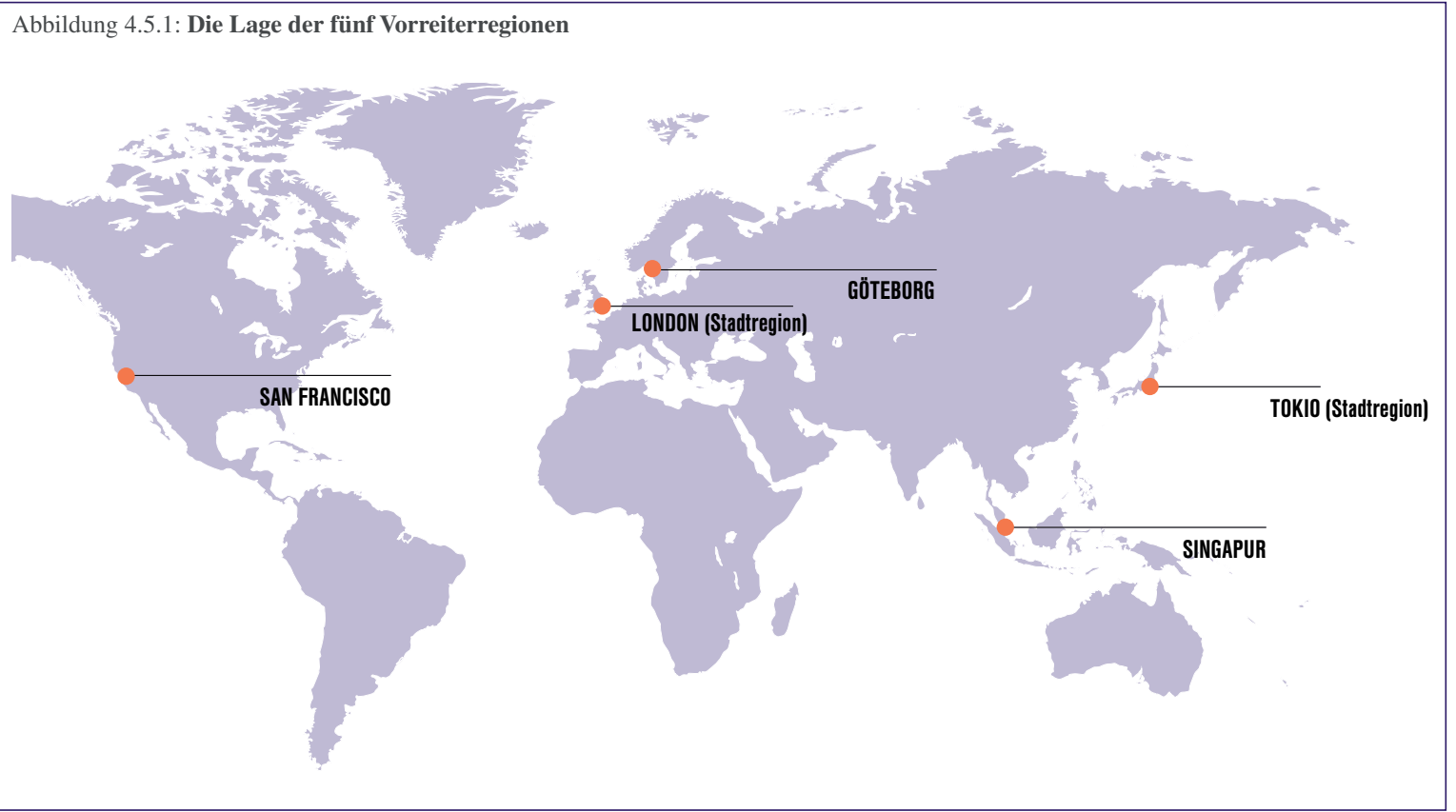

Quelle: AVENUE21 
- Die Dimension „Regime“ schließt die vorherrschenden Praktiken, Regeln etc. eines Landes ein.

- Die Dimension „Nische“ umfasst ,geschützte Räume“ wie Forschungslabore, Start-ups etc., bei denen mit Innovationen experimentiert wird.

Angelehnt an diese beiden Theorien wurden zur Untersuchung und vergleichenden Bewertung der Vorreiterregionen vier analytische Kategorien entwickelt:

1 „Treiber" beziehen sich einerseits auf den Handlungsdruck (,,pressure“), der sich aus gesellschaftlichen und ökologischen Herausforderungen ergibt (,landscape“), und andererseits auf den Druck aus der „Nische“.

2 "Innovationsnetzwerke“ beziehen sich auf die beteiligten Schlüssel-AkteurInnen und Allianzen, die gemeinsam den Fortschritt und Wandel planen und einleiten (,Regime“ und „Nische“).

3 „Entwicklungsnarrative“ beziehen sich auf Visionen und Leitbilder, die in korporativ-politischen Aushandlungsprozessen entstanden sind (Narrative; s. Kap. 4.6);

4 „Transition-Initiativen“ sind daran anschließende Handlungsstrategien und Programme, die eine Orientierung für eine gemeinsame Handlung (z. B. in Form von Roadmaps) geben sollen.

$\mathrm{Zu}$ den vier analytischen Kategorien wurden vier Forschungsfragen formuliert. In allen Fragen lag der Fokus auf dem Zusammenspiel von avM-Strategien und den langfristigen Entwicklungsplänen für Stadtentwicklung und Verkehrsplanung in der jeweiligen Vorreiterregion (s. Abb. 4.5.2).

\section{ZUSAMMENFASSUNG DER ERGEBNISSE: KONZEPTION VON AUTOMATISIERTER UND VERNETZTER MOBILITÄT AUS DER PERSPEKTIVE VON STÄDTEN/STADTREGIONEN}

In einer kompakten Weise werden hier die Zugänge der fünf bestimmten Vorreiterregionen (Städte/Stadtregionen) mit Bezug auf die vier analytischen Kategorien reflektiert: (1) Treiber, (2) Innovationsnetzwerke, (3) Entwicklungsnarrative und (4) Transition-Initiativen.

\section{- SAN FRANCISCO (USA)}

Treiber: Die aktuellen städtischen Herausforderungen in San Francisco sind ein akuter Wohnungsmangel, ein fragmentiertes öffentliches Verkehrssystem, ungleiche Wachstumsmuster zwischen den Bezirken, eine wachsende Nachtwirtschaft und eingeschränkte Mobilitätsmöglichkeiten insbesondere für einkommensschwache Bevölkerungsgruppen, SeniorInnen und Menschen mit Behinderungen (SFMTA 2016a und 2016b, S. 1; SFMTA 2018). Insbesondere die Tech Giants des Silicon Valley drängen darauf, ihre Technologien auf den StraBen von San Francisco zu testen.

Innovationsnetzwerke: Die San Francisco Municipal Transportation Agency (SFMTA) ist in San Francisco Hauptakteurin in der Unterstützung des automatisierten Verkehrs. Es wurde ein Begegnungsort, eine Agentur und ein Ideen-Inkubator namens Smart City Institute (auch Superpublic genannt) eingerichtet (ATCMTD, City of San Francisco 2016, S. 14). Durch den „Community-Guided Engagement Plan“"wurde ein Rahmen geschaffen, um unterschiedliche Kooperationen und Allianzen zwischen verschiedenen Sektoren und AkteurInnen (,cross-sector collaboration“) zu erreichen. Die Stadt hat zudem eine Allianz mit dem World Economic Forum samt besonderem Fokus auf die avM gebildet.

Abbildung 4.5.2: Kategorien und zentrale Forschungsfragen im Rahmen der Analyse der Vorreiterregionen

\begin{tabular}{|c|c|}
\hline ANALYTISCHE KATEGORIEN & FORSCHUNGSFRAGEN \\
\hline $\begin{array}{r}\text { Treiber: } \\
\text { Herausforderungen und Erwartungen }\end{array}$ & $\begin{array}{l}\text { - Was bewegt Städte/Regionen (Politik, Verwaltung), in avM zu investieren? } \\
\text { - Welche Treiber entstehen aufgrund von Handlungsdruck aus „Landscape“ oder „Nische“? }\end{array}$ \\
\hline $\begin{array}{l}\text { Innovationsnetzwerke: } \\
\text { AkteurInnen und Kooperationen }\end{array}$ & $\begin{array}{l}\text { - Wer sind die relevanten AkteurInnen in einer Stadt/Stadtregion, die sich für die } \\
\text { Einführung und Verbreitung des avV einsetzen? } \\
\text { - Welche Kooperationen gibt es? Welche Allianzen wurden gebildet? } \\
\text { - Welche Schritte wurden unternommen, um Fortschritt und Wandel hin zu avM zu } \\
\text { erreichen? }\end{array}$ \\
\hline $\begin{array}{l}\text { Entwicklungsnarrative: } \\
\text { Visionen und Leitbilder }\end{array}$ & $\begin{array}{l}\text { - Welche Zukunftsvorstellungen wurden gemeinsam formuliert? Welche strategischen } \\
\text { Ziele und Leitbilder wurden daraus abgeleitet? } \\
\text { - Welche Erzählungen werden bevorzugt, um avM attraktiv zu machen (,,basket of images“)? }\end{array}$ \\
\hline $\begin{array}{l}\text { Transition-Initiativen: } \\
\text { Strategien und Programme }\end{array}$ & $\begin{array}{l}\text { - Wie ist der mögliche Übergang zu avM vorgesehen? Welche Ziele und Strategien gibt es } \\
\text { hierzu? Welche Investitionsprogramme wurden vereinbart? } \\
\text { - Wie wurden die Strategien und Umsetzungen formuliert (z.B. als Roadmaps)? }\end{array}$ \\
\hline
\end{tabular}


Entwicklungsnarrative: Die ersten beiden Narrative von avM beziehen sich hauptsächlich auf die Sicherheit (,Vision Zero SF“) und auf verbesserte Mobilitätsoptionen für ökonomisch schwächere Gruppen (,Transportation is the greatest equalizer of all“). Mit dem dritten Narrativ wird die avM direkt angesprochen. Dabei wird eine mittel- und langfristig orientierte $\mathrm{Zu}$ kunftsvorstellung, getragen von Shared-Electric-Connected-Automated Vehicles (SECAV), entworfen.

Transition-Initiativen: Die Heimat der Sharing Economy hat es sich zur Aufgabe gemacht, ein hohes Maß an geteilten Mobilitätsformen einzuführen, zu erproben und später im breiten Mobilitätsangebot zu verankern. Um dieses langfristig angesetzte Ziel zu erreichen, wird in starkem Maße auf den Einsatz von avF aufgebaut. Der Übergang zu SECAV wurde in Form eines Stufenplans konzipiert. Wichtigstes mittelfristiges Ziel ist die Rückgewinnung von Raum in der Innenstadt (bisher Parkraum), um z. B. mit steigender Verdichtung mehr Wohnraum schaffen zu können. In keiner anderen Region der Welt wurden bislang so viele reale und virtuelle Kilometer im automatisierten Verkehr zurückgelegt wie in Kalifornien (US Senate Hearing 2018).

\section{- LONDON UND STADTREGION (UK)}

Treiber: Aktuelle Herausforderungen für die Stadtentwicklung in London sind die schnell wachsende Bevölkerungszahl in der Region, auf den zentralen Verkehrsrouten zunehmende Staubildungen, eine sich verschärfende soziale Ungleichheit und die akute Notwendigkeit, das schnelle städtische Wachstum durch Dezentralisierung zu kompensieren (GLA 2015). Die vielfach geteilte Erwartung der technisch-ökonomischen Vorteile der Entwicklung und des Einsatzes von avF ist der wichtigste Treiber für Investitionen in Forschung und Anwendung von avF in Großbritannien.

Innovationsnetzwerke: In Großbritannien gibt es zahlreiche Universitätsinstitute, einflussreiche nichtuniversitäre Forschungsorganisationen und Think Tanks, die zum lokalen und nationalen Diskurs und zur Förderung und Entwicklung von avF stark beitragen. Das Department for Transport (DfT) und das Department for Business, Energy \& Industrial Strategy (BEIS) Großbritanniens haben im Jahr 2017 zusammen das Centre for Connected and Autonomous Vehicles (CCAV) ins Leben gerufen (UK Parliament 2017). Das CCAV ist die zentrale Anlaufstelle für die Entwicklung des avV in Großbritannien.

Entwicklungsnarrative: Die wichtigsten Entwicklungsnarrative und Strategien zur Zukunft der Mobilität und die darin eingebetteten Visionen zur avM konzentrieren sich auf ökonomische Wettbewerbsvorteile, auf Gesundheit und Wohlbefinden, auf soziale Inklusion, auf ,gutes“ Wachstum und auf die Vorstellungen einer polyzentrischen Stadt (GLA 2017, NLA 2017, TfL 2017).

Transition-Initiativen: Die langfristige Stadtentwicklungsstrategie Londons basiert auf zwei Säulen. Zum einen wird auf die Dezentralisierung des Wachstums gesetzt und zum anderen auf die Gesundheit und das Wohlbefinden der BürgerInnen (TfL 2017) . Eine detailreiche Roadmap zur Entwicklung des avV in Großbritannien wurde nach umfassenden öffentlichen Konsultationen formuliert (DfT 2015). In diesem Zuge wurde ein besonderes Verfahren zur Auswahl von Förderprojekten zur Entwicklung und Erprobung von avF umgesetzt. In zahlreichen Projekten wurde untersucht, wie BürgerInnen die avM im Alltag einschätzen und wie sie damit umgehen.

\section{GÖTEBORG (SCHWEDEN)}

Treiber: Die Herausforderungen in Göteborg sind eine steigende Bevölkerungszahl, wachsende ökonomische und soziale Ungleichheit, zunehmender Warenverkehr (der größte Hafen Skandinaviens befindet sich in Göteborg), die Auswirkungen des Klimawandels auf zahlreiche städtische Bezirke (steigender Meeresspiegel) sowie eine wachsende wissensbasierte Wirtschaft, die neue Arten von sozioökonomischen Kommunikationsräumen benötigt. Die Präsenz einer Reihe von Unternehmen (wie die Volvo-Gruppe) hat bei der Stadt Erwartungen geweckt, dass durch Investitionen in Forschung und Entwicklung von avF die Stadt ökonomische Wettbewerbsvorteile erreichen kann.

Innovationsnetzwerke: Die Kooperations- und Kollaborationsplattform Drive Sweden ist die wichtigste Organisation, die in Schweden eingerichtet wurde, um die Entwicklung von avF öffentlich zu unterstützen (Drive Sweden 2018). Mit sogenannten „Co-Creative Labs“ (SAFER 2017) versucht die Stadtplanung in Göteborg, die Bevölkerung einzubinden, um gemeinsam eine bürgerschaftlich getragene Perspektive für die avM zu entwickeln.

Entwicklungsnarrative: Das zentrale Stadtentwicklungsnarrativ in Göteborg ist die „Compact City“ (City Planning Authority 2014). Dabei geht es zum einen um die Nachverdichtung der Stadt und zum anderen darum, Göteborg als Standort in einer wissensbasierten Wirtschaft zu entwickeln und zu stärken. Die Bevölkerung wird für einen Wandel von städtischer Mobilität und Verkehr sensibilisiert (,A completely new type of mobility“).

Transition-Initiativen: Göteborg soll eine Stadt der kurzen Wege (,closely-connected city“) werden (Urban Transport Committee 2014). Drive Sweden fördert verschiedene kooperative Forschungs- und Entwicklungs- 
projekte wie den Einsatz von selbstfahrenden Shuttlebussen, die für eine kompakte Stadt geeignet sind. Eine interaktive und ständig fortschreitende Roadmap zur Entwicklung der avM in Schweden auf der Website von Drive Sweden informiert interessierte AkteurInnen über den Stand der Entwicklung und mögliche Phasen des Übergangs zum vollautomatisierten Fahren. Ein großer Schritt für die Stadt Göteborg und ihren Automobil- und Kommunikationssektor war der Bau von Asta Zero, einem international bedeutenden Testgelände für avF.

\section{- TOKIO UND STADTREGION (JAPAN)}

Treiber: Die Herausforderungen in Tokio (und Japan insgesamt) sind eine dramatisch schrumpfende und alternde Bevölkerung, ein akuter Arbeitskräftemangel und ein stagnierendes Wirtschaftswachstum (Funabashi 2018, IPSS 2017). Darüber hinaus war und ist auch die Ausrichtung der Olympischen Spiele im Jahr 2020 in Tokio ein Treiber für die rasche Entwicklung von avF.

Innovationsnetzwerke: Das Council for Science, Technology and Innovation hat mit dem SIP („Cross-Ministerial Strategic Innovation Promotion Program“) - und der Unterstützung des Premierministers - eine besondere Art von horizontaler bereichsübergreifender Organisationsstruktur geschaffen (Amano \& Uchimura 2016, 2018; SIP 2017). So wurde ein Prozess gestartet, um die Abschottung institutioneller ,Silos“ zu überwinden. Ein spezifischer Bereich dieses Programms, das SIPADUS (Automated Driving for Universal Services), ermöglicht sowohl die Koordinierung von Forschung und Entwicklung (SIP-ADUS für die avM) als auch die Orchestrierung der „Triple-Helix“ in Japan zwischen AkteurInnen der Regierung (bereichsübergreifend), der Industrie und Forschungsinstitutionen (Amano \& Uchimura 2016, 2018; SIP-ADUS 2016).

Entwicklungsnarrative: Mit „Mobility bringing everyone a smile“ (Kuzumaki 2017) möchte die Stadtregierung vor allem die Inklusion von SeniorInnen erreichen. Die beiden Narrative „World's safest and smoothest road traffic system“ (Prime Minister's Cabinet 2017) und „World's most advanced IT nation“ sprechen die Wirtschaft an, während sich die Roadmap ,Society 5.0“ auf die gegenwärtige öffentliche Diskussion in Japan zur Zukunft der Gesellschaft im Zeitalter der Digitalisierung, der Roboter und der künstlichen Intelligenz bezieht (FIRST 2018). Der Aspekt „Safety“ betrifft in Japan die Erfahrungen einer ständig drohenden Gefahr durch starke Erdbeben; so wurden schon bisher besondere Sicherheitssysteme im ÖV installiert.

Transition-Initiativen: Tokios Entwicklung bis zum Jahr 2020 wird durch mehrere Aktionspläne gerahmt. Die Hauptidee ist, die Megalopolis Tokio mit zahlreichen kompakten Verkehrsdrehscheiben (,compact city hubs“) auszustatten (MLIT 2013, Tokyo Metropolitan Government 2016). Die beiden wichtigsten gesellschaftlichen Fragen, für die von der avM Lösungen erwartet werden, sind zum einen die Zunahme der Zahl der alternden Bevölkerung und zum anderen die sinkende Zahl an Arbeitskräften. Die meisten avM-Projekte sind derzeit darauf ausgerichtet, Tokio mit seinen Vororten bis hin zum ländlichen Raum zu verbinden (Prime Minister's Cabinet 2017). Unter der koordinierenden Hand der bereichsübergreifenden SIP-ADUS hat Tokio die detaillierteste avM-Roadmap aller hier dargestellten Vorreiterregionen erstellt. Sie enthält alle Ziele, Strategien und Konzepte für den Einsatz automatisierter und vernetzter Fahrzeuge, einschließlich eines Zeitplans für die Umsetzung und Markteinführung in Japan bis zum Jahr 2030. Ein besonderes Merkmal der Strategie in Japan ist die sukzessive Erstellung einer dynamischen, landesweiten digitalen Karte der Straßenräume (Koyama 2015).

Die älter werdende Bevölkerung und der damit einhergehende dramatische Rückgang der Bevölkerung im ländlichen Raum gehören zu den dominierenden Themen in Japan. In der langfristigen Vision ,The Grand Design 2050“ (MLIT 2013) geht es um die Verdichtung von kleineren Städten und Gemeinden im suburbanen und ruralen Japan (Funabashi 2018). Die meisten avM-Projekte sind derzeit auf diese Themen ausgerichtet.

\section{- SINGAPUR}

Treiber: Städtische Herausforderungen sind die akute Landknappheit in Verbindung mit der wachsenden Bevölkerungszahl sowie steigende Forderungen der Bevölkerung nach besseren Mobilitätsmöglichkeiten, der Neu- und Ausbau von Stadtgebieten (,New Towns“) sowie ein Mangel an Facharbeitskräften (LTA 2013, 2018; Loo 2017; Human Resources 2018). Weitere Treiber sind der Ausbau der globalen Wettbewerbsfähigkeit und der Erhalt der Standortqualität.

Innovationsnetzwerke: Das ressortübergreifende Committee on Autonomous Road Transport for Singapore (CARTS) wurde in der Stadtverwaltung von Singapur geschaffen, um sich ausschließlich mit der Erprobung und Umsetzung von avF und avM in Singapur auseinanderzusetzen (Huiling \& Goh 2017, MOT 2017). Der Ausschuss wird von drei „working groups“ (sektorenübergreifend und transdisziplinär) begleitet, die für die Erstellung von mittel- und langfristigen Visionen für die avM in Singapur verantwortlich sind und die Rahmenbedingungen für möglichen Anwendungen (Kosten, Regulierungsrahmen etc.) prüfen.

Entwicklungsnarrative: Die drei wichtigsten Entwicklungsnarrative sind die alles umfassende Digitalisierungsstrategie Singapurs „Smart Nation“, die Vision 

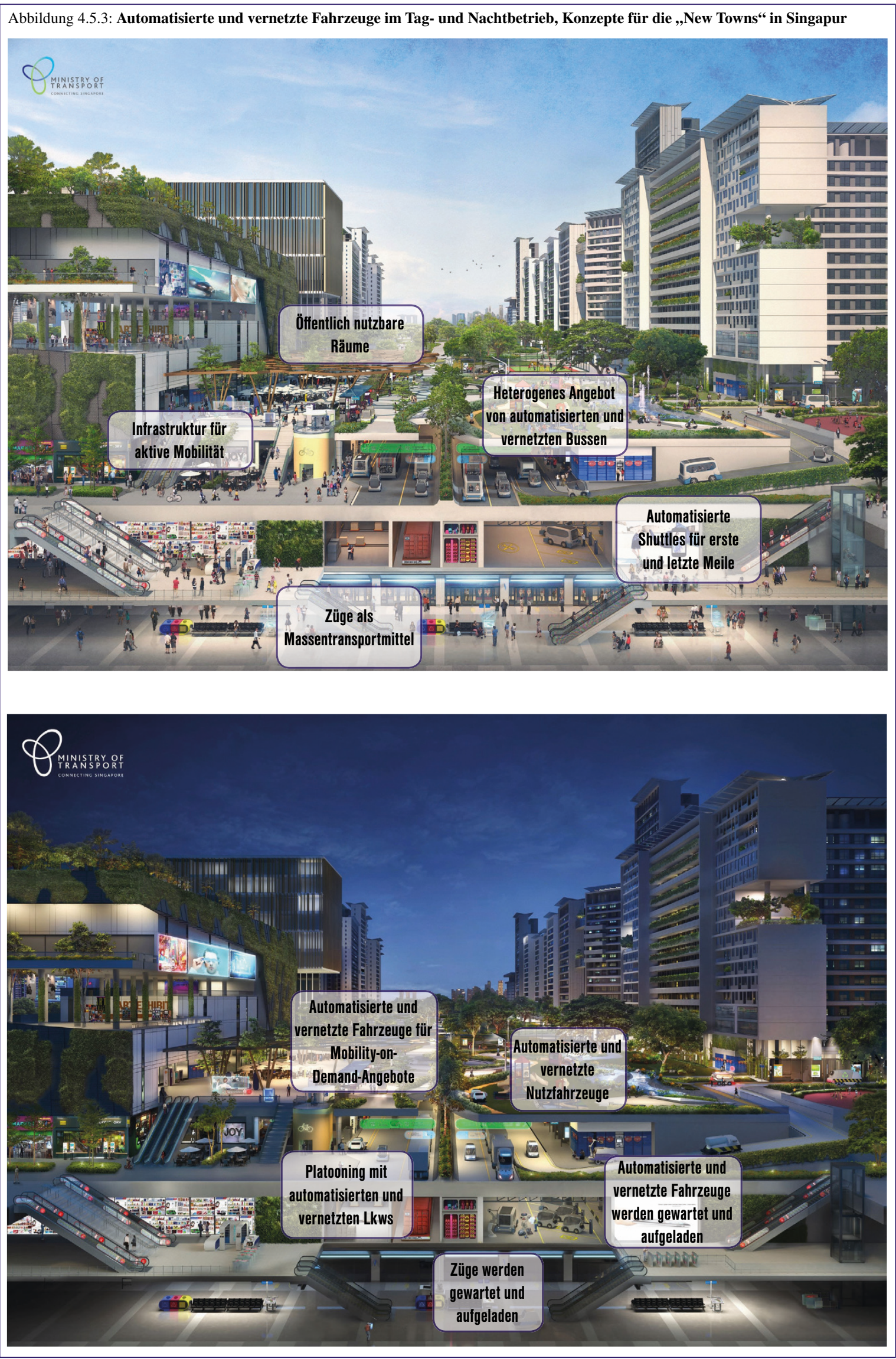

Quelle: Land Transport Authority LTA, von AVENUE21 ergänzt 
der autofreien Stadt „Car-lite City“ (LTA 2018) und das Leitbild der lebenswerten Stadt „Livable City“ (CLC 2014, Smart Nation Singapore 2018). Der Innovationsdiskurs in Singapur ist davon geprägt, wie neue Technologien mit einem identitätsstiftenden und ,emotionalen“ Urbanismus vereinbar sein können („Can you love a smart city?"). Um die Zahl der zugelassenen Autos zu begrenzen, werden seit Februar 2018 neue Autos in Singapur nur dann zugelassen, wenn sie ein bestehendes Auto ersetzen.

Transition-Initiativen: Verglichen mit allen anderen Vorreiterregionen genießen avF in Singapur den höchsten Grad der Integration in die Stadtentwicklung. Die zentralisierte Regierung des Stadtstaates Singapur hat seit 50 Jahren das Ideal einer integrierten Planung durch verschiedene Instrumente der interinstitutionellen Zusammenarbeit verfolgt (URA 2016). Auf der Grundlage einer intensiven sektorenübergreifenden Kooperation (inklusive der Kooperation mit Investoren) können Standorte und Anwendungsfelder (Use Cases) für avF sachgerecht und zielgerichtet geplant und umgesetzt werden. Zwei neue hochverdichtete Siedlungsgebiete (,New Towns“), die bis 2024 fertiggestellt werden sollen, sind bereits für diverse standortspezifische Anwendungen von avF für Peak- und OffPeak-Stunden sowie Tag- und Nachtbetrieb vorgesehen (s. Abb. 4.5.3). In Singapur wird zudem intensiv an Bildungsformaten und -programmen für die Ausbildung und Weiterbildung von Arbeitskräften im Bereich der avM gearbeitet.

\section{CONCLUSIO: „AUTOMATED AND CONNECTED MOBILITY FOLLOWS (INNOVATION) NARRATION“}

Die Ergebnisse der Studie deuten darauf hin, dass, obwohl die Steigerung von Sicherheit, Verkehrseffizienz, ökonomische Vorteile oder die Einsparung von Parkraum in der Regel die meistgenannten Argumente für den Einsatz von avF in der Stadt sind, die lokalen Entwürfe und avM-Strategien in der Realität dennoch überraschend divers sein können. Im Zuge dieser Diversität zeigen sich sogar Gegennarrative zum bestehenden ökonomischen Mainstream. Die Fallbeispiele zeigen, dass Technologie für avF und avM nicht deterministisch und diesbezügliche Strategien das Ergebnis komplexer Aushandlungsprozesse sind. Es ist auch wichtig, anzumerken, dass sich alle Städte auf eine gesamtsystemische Übergangszeit von mehreren Jahrzehnten vorbereiten. Die meisten von ihnen haben jedoch besondere Stadträume ins Auge gefasst, wo sie avF mit niedrigeren Automatisierungsstufen viel früher einsetzen können.

Die vier analytischen Kategorien (1) Treiber, (2) Innovationsnetzwerke (3) Entwicklungsnarrative und (4) Transition-Initiativen zeigen in allen fünf Vorreiterregionen, dass für komplexe Übergangsprozesse zu
avM-basierten Lösungen die Intensität und Färbung des Innovationsdiskurses vor Ort entscheidend ist. Es ist auch zu erkennen, dass die Einführung und Verbreitung von avF nur Teil der digitalen Transformation aller Lebensbereiche ist (,Vierte industrielle Revolution“). Angesichts der weitreichenden Konsequenzen der digitalen Transformation ist die entscheidende Frage für den Einsatz von avF folglich nicht, ob die Stadt für avF bereit ist oder ob avF für den Einsatz in der Stadt bereit sind, sondern wie die AkteurInnen der Stadt an sich und in Kooperation mit anderen einen Diskurs über nachhaltige Stadtentwicklung im digitalen Zeitalter führen (Co-Produktion in Netzwerken). Die alltagsweltliche Umsetzung der avM folgt dann jener im Diskurs der etablierten Narration. 
4.6

\section{DIE AUSHANDLUNG EINER VORHERRSCHENDEN NARRATION ZUR AUTOMATISIERTEN UND VERNETZTEN MOBILITÄT IN EUROPA}

Einblick in die Dissertation von Andrea Stickler

Welche Rolle die Automatisierung im Verkehrssystem einnehmen wird, ist nicht nur durch die technologischen Möglichkeiten bestimmt, sondern ist auch wesentlich davon abhängig, welche Diskurse und Narrationen ${ }^{1}$ sich in der Öffentlichkeit über die avM festigen. Großen Einfluss auf diese Zukunftsvorstellungen und damit auch die Akzeptanz von avF üben vermittelte Botschaften von verschiedenen Wirtschaftszweigen, Medien, Forschung, Film oder Kunst und eben auch der Politik aus (Diehl \& Diehl 2018, Manderscheid 2018, Berscheid 2014). Der politische Diskurs nimmt jedoch eine besondere Rolle ein, da dieser in den letzten Jahren äußerst brisant vorangeschritten ist, zu bestimmten politischen Handlungen führt bzw. führte und damit auch wesentlich über die Bedingungen des Einsatzes der Technologie mitbestimmt (durch zirkulierendes Wissen, Test-Beds und Best-Practice-Beispiele, Förderinstrumente, Regulationen etc.). Daher stellt sich die Frage, in welche Richtung sich die Politik (AkteurInnen, Institutionen, Prozesse und Inhalte) im Zuge der Einführung von avF hin bewegt und welcher politisch-planerische Rahmen damit vordefiniert wird, der letztlich auch auf künftige Steuerungsmöglichkeiten wirkt. $^{2}$

Wenngleich die Hoffnungen auf technologische Innovationen im Verkehrswesen im politischen Diskurs bereits seit mehreren Jahren stark präsent sind (u. a. Europäische Union 2011), so sind die Erwartungen an die positiven Effekte von avF relativ neu. Die Verkehrsministerien der G7-Staaten haben im Jahr 2015 erstmals eine Deklaration zur strategischen Förderung von avM herausgegeben. Auf EU-Ebene wurde im April 2016 mit der „Deklaration von Amsterdam“ ein Konsens zwischen den 28 Verkehrsministerien zur gemeinsamen Förderung der Einführung von avF gebildet und die Europäische Kommission zu einer europäischen Strategie zur avM aufgefordert (European Commission 2016). Im Kontext dieser Zuversicht zur Durchsetzung der Automatisierung und Vernetzung der Mobilität und zu den erwarteten positiven Effekten formieren sich bestimmte Narrationen darüber, wie avF in Europa eingesetzt werden sollen und wirken werden.

Das politisch-diskursive Spielfeld kennzeichnet sich gerade im Bereich der Verkehrspolitik durch konkurrierende Ideen, Problemdeutungen und Interessen, aber auch durch unterschiedliche Kommunikations- und Machtressourcen verschiedener StakeholderInnen. Was als gesichertes Wissen zur avM gilt, steht dabei in unmittelbarem Zusammenhang mit Machtfragen, denn die Fähigkeit und Durchsetzungskraft über eine vorherrschende Narration auf politischer Ebene mitzubestimmen, differiert zwischen den unterschiedlichen

Abbildung 4.6.1: Narrationen zur automatisierten und vernetzten Mobilität

Wahrgenommener Handlungsdruck

(aufgrund von technologischer Entwicklung, internationalem Wettbewerb, politisch wahrgenommenen Problemen der Automobilität)

Machtverhältnisse

(Involvierte StakeholderInnen und ihre Entscheidungskraft)

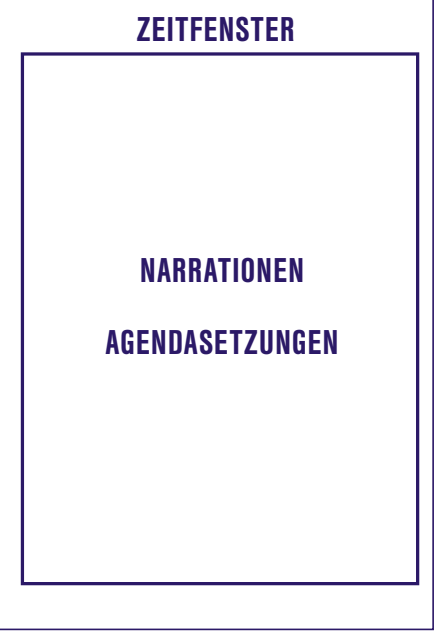

Quelle: AVENUE21 nach Kingdon (1986) 


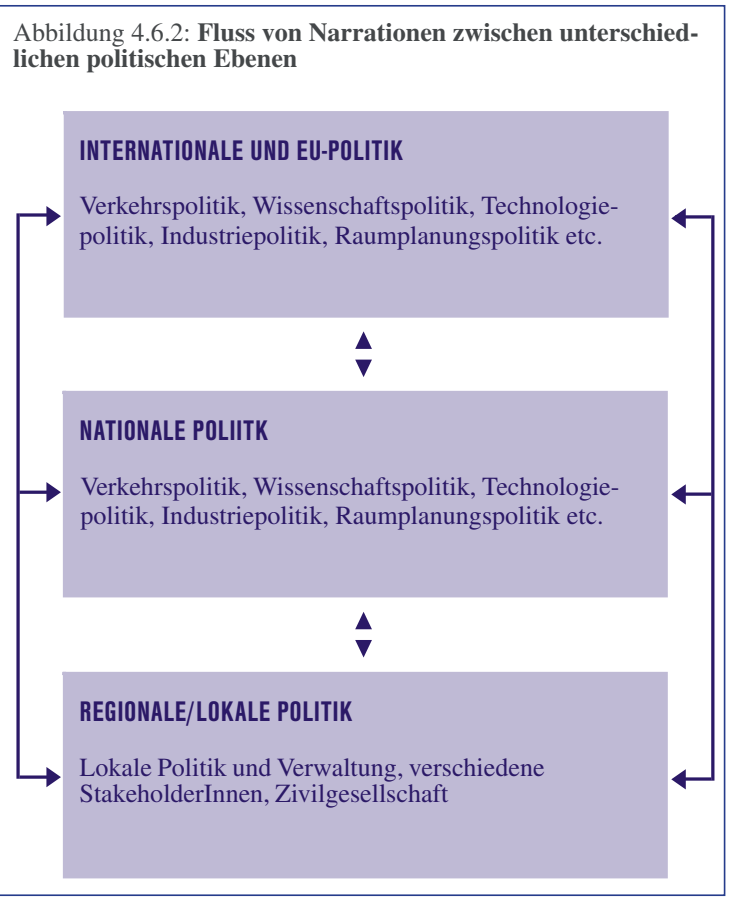

Quelle: AVENUE21

StakeholderInnen enorm. Dem Multiple-Stream-Ansatz folgend (Kingdon 1986), sind neben den Machtverhältnissen auch der wahrgenommene Handlungsdruck sowie bestehende Verkehrspolitiken, -lösungen und -infrastrukturen für politische Narrationen und Agendasetzungen in einem spezifischen Zeitfenster relevant (s. Abb. 4.6.1).

In politischen Debatten zur avM werden zweifellos relevante gesellschaftliche Herausforderungen wie Klimaschutz, Sicherheit, der Platzverbrauch von Pkws und Chancen für mobilitätseingeschränkte Personen aufgegriffen und öffentlich zur Sprache gebracht. Jene Probleme werden in diesem Zusammenhang jedoch einer Konstruktionsarbeit unterzogen, für die dann in einer Kooperation zwischen Politik, verschiedenen Industrien und Forschungseinrichtungen spezifische technologische Lösungen gesucht und entwickelt werden. Hoffnungen über die Zukunft und Brüche mit der Vergangenheit bilden in diesem Zusammenhang eine wesentliche Grundlage für die Aushandlung einer vorherrschenden Narration zur avM. Vor allem hinsichtlich des Stellenwerts der Automobilität ist der politische Diskurs heute noch stark gespalten und auch historisch haben sich unterschiedliche Phasen der Befürwortung und Kritik an der Automobilität abgewechselt (Paterson 2007).

Im Feld der Verkehrspolitik ist jedenfalls die nicht vorhandene Nachhaltigkeit des heutigen Autoverkehrs weitgehend unumstritten, weshalb sich der Diskurs innerhalb der EU zur avM auch zunehmend an umweltpolitischen Argumentationsmustern orientiert. Eine ökologische Modernisierung (Bemmann et al. 2014) des Automobils soll helfen, die positiven synergetischen Effekte von Fahrzeugautomatisierung, Elektrifizierung und Konnektivität zu nutzen, gleichzeitig die Wettbewerbsfähigkeit der Wirtschaft (und Automobilindustrie) sowie die europäische Integration zu stärken. Damit verfestigt sich die Narration einer überwiegenden technikgläubigen, wachstumsorientierten Transformation der Automobilität mit avF durch eine infrastrukturelle Priorisierung von wichtigen Verkehrsachsen innerhalb der EU (hier insbesondere der C-ITS-Korridor von Rotterdam nach Frankfurt und Wien).

Die Narration der EU zur avM, die über Politikprogramme, politische Gremien, Veranstaltungen und Medien transportiert wird, verbreitet sich als neue Art des Denkens und Handelns in verschiedenen Räumen und Institutionen (weshalb der Fluss der Narrationen zwischen unterschiedlichen politischen Ebenen und Politikfeldern zu betrachten ist, s. Abb. 4.6.2).

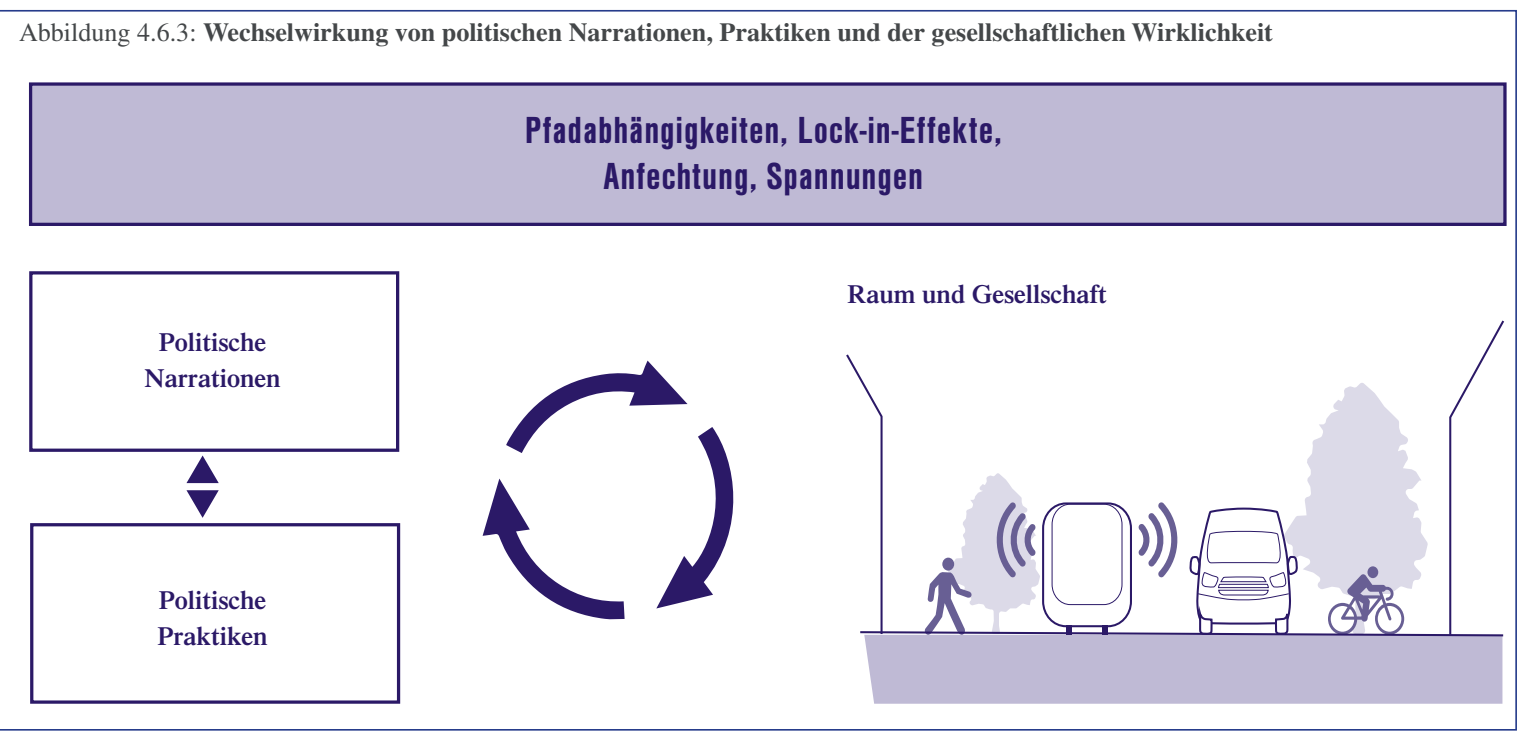

Quelle: AVENUE21 
Narrationen auf EU-Ebene treten dabei in Auseinandersetzung mit anderen (inter)nationalen Vorstellungen zur avM, üben Druck auf unterschiedliche politische Ebenen aus und werden teilweise von nationalen oder lokalen Institutionen aufgenommen und ,übersetzt“ (Clarke et al. 2015). Die Übersetzung ermöglicht mithin eine gänzlich neue Aushandlung des Themas, mit neuen Spannungen, Risiken und Chancen. Bestimmte Aspekte können betont oder vernachlässigt werden, letztlich können auch unerwartete Effekte aufgrund von Pfadabhängigkeiten, Lock-in-Effekten, Anfechtung und Spannungen folgen (s. Abb. 4.6.3). Daher ist es notwendig, um die Beziehungen zwischen den politischen Narrationen und den bestimmten politisch-planerischen Handlungen zu verstehen, konkrete räumliche Gegebenheiten in die Betrachtung miteinzubeziehen (Jäger 2015, S.112-113). Erst dadurch wird deutlich, dass politische Diskurse und Narrationen zwar auf die Verkehrswende wirken werden, jene die Verkehrswende aber nicht vorgeben, ist doch die Transformation der Automobilität eingebettet in komplexe gesellschaftliche, politökonomische und historisch gewachsene Zusammenhänge (Urry 2004, Paterson 2007). Dennoch können politische Diskurse einen wesentlichen Beitrag zur Verkehrswende leisten, da an sie gewisse Diskurspraxen gekoppelt sind, die dann durchwegs struktur- und raumwirksam werden können.

Die technik- und wachstumsorientierte Vorstellung von avM innerhalb der EU wird heute bereits durchaus angezweifelt. Gerade auf lokaler/städtischer Ebene werden die negativen Effekte der Automobilität sowie deren Wachstumsgrenzen am deutlichsten spürbar, weshalb ein weiteres Verkehrswachstum mit avF sehr kontrovers diskutiert wird. Auch empirisch und theoretisch kann eine wachstumsorientierte Transformationsnarration angefochten werden: Statistiken deuten auf ein reges Verkehrswachstum im Straßenverkehr hin (Umweltbundesamt 2018, Statistik Austria 2018), das künftig an seine (ökologischen) Grenzen stoßen wird. Gesellschaftliche Prozesse wie Individualisierung, Flexibilisierung und soziale Beschleunigung (Rosa 2013, Honneth 2016) sowie die Dynamik des globalen Wirtschaftssystems (Boltanski \& Chiapello 2001, Schwedes 2017) wirken einer umweltschonenden Mobilität kulturell und strukturell eher entgegen. Vor dem Hintergrund dieser Tendenz zur Verkehrssteigerung sowie zu einer wachstumsorientierten Narration zum avV könnten viele negative Effekte der Automobilität (Platzverbrauch, Beeinträchtigung von Fuß- und Radverkehr, Lärmemissionen etc.) vor allem in Städten verschärft oder konserviert werden.

Dennoch wird in einigen Diskursen auch das Potenzial einer tiefgreifenden Transformation mit avF erkannt, denn Fortschritte in der Automatisierung und Vernetzung der Fahrzeugtechnologie können nicht nur das Auto als technisches Gerät verändern, sondern auch die Bedeutung des Pkws in Privatbesitz und letztlich auch wie und warum Individuen mobil sind (Kellerman 2018, Canzler \& Knie 2016). Diese kultur- und wachstumskritischen Diskurse werden jedoch in politischen Debatten auf europäischer Ebene oftmals vernachlässigt. Für eine stärkere Politisierung der avM wäre es jedoch wichtig, dass vorherrschende und subalterne Narrationen stärker in Auseinandersetzung treten. Dies erfordert jedoch, dass auf politischer Ebene über eine ökologische Modernisierung des Automobils hinweg gedacht wird, gegensätzliche Vorstellungen zur $\mathrm{Zu}$ kunft mit avF diskutiert und unterschiedliche Möglichkeiten des Einsatzes von avF stärker gesellschaftspolitisch ausgehandelt werden.

Zusammenfassend ist festzuhalten, dass gerade aufgrund der konkurrierenden Problemdeutungen und Interessen in der heutigen Verkehrspolitik gegenwärtige politische Narrationen zur avM kritisch herausgefordert werden sollten, denn:

- In der EU bildet sich im Spannungsfeld unterschiedlicher Akteursinteressen eine bestimmte Narration davon heraus, wie avF bestmöglich eingesetzt werden sollten. Dies führt zu veränderten gesetzlichen Rahmenbedingungen und Förderstrategien als politische Praktiken, die wesentlich auf den künftigen Einsatz von avF wirken werden.

- Die Narrationen und politischen Praktiken der EU erlangen auf lokaler Ebene eine andere Bedeutung, werden durchaus auch angezweifelt und neu ausgehandelt. Dieser Aushandlungsprozess sollte bewusst gestärkt und für unterschiedliche Interessensgruppen geöffnet werden.

- Forschungsarbeiten können zur Aufdeckung der vorherrschenden Machtverhältnisse beitragen, die den Diskurs zur avM prägen und als „Frühwarnsysteme“ für mögliche unerwartete Konsequenzen dienen.

1 Mit dem Begriff der Narration wird hier die kommunizierte und inszenierte Erzählung bzw. der Mythos über avF und avM verstanden. Diese Narrationen bezeichnen Regelsysteme, die bestimmte Wissensformen und Bedeutungen hervorbringen und damit den öffentlichen und/oder politischen Diskurs strukturieren (Viehöver 2001, S.177-178).

2 Konkret wird der Frage nachgegangen, wie die avM von unterschiedlichen StakeholderInnen als Wissensobjekt hervorgebracht wird. Dazu wird diskurstheoretisch vorgegangen und auch methodisch auf Diskurstheorien, wie beispielsweise von Keller (2004) und Jäger (2015), Bezug genommen. 
4.7

\section{PLANUNGSANSÄTZE FÜR EINE PROAKTIVE GESTALTUNG URBANER ZUKÜNFTE MIT AUTOMATISIERTEN UND VERNETZTEN FAHRZEUGEN}

Einblick in die Dissertation von Emilia Bruck

Wenngleich automatisierte und vernetzte Mobilität zunehmend vor dem Hintergrund bestehender Stadtentwicklungsziele diskutiert wird (Heinrichs et al., 2019), wurden geeignete Planungsansätze für die Steuerung der städtischen Integration von avF bislang unzureichend behandelt (Guerra 2016, Fraedrich et al. 2018). Bis auf wenige Ausnahmen (Kap. 4.5) lag aus Sicht der städtischen Planungsbeauftragten bis zuletzt kein ausreichendes Wissen über die städtebaulichen und siedlungsstrukturellen Folgen der avM vor, um Entwicklungsprioritäten festzulegen und Investitionen entsprechend lenken zu können (Fraedrich et al. 2018). Es bestehen Unsicherheiten hinsichtlich infrastruktureller, stadträumlicher und gesellschaftlicher Auswirkungen, da bislang der lokale Nutzen der avF im Sinne von umwelt- und stadtverträglicher Mobilität nicht ausreichend geprüft werden konnte. Bedenkt man jedoch, dass die Art und Weise sowie das Ausmaß möglicher Folgen der avM teils erst nach deren Eintreten erkenn- bar sein werden (Guerra 2016), bedarf es seitens der Stadtplanung einer reflektierten Auseinandersetzung und frühzeitigen Mitgestaltung, um als treibende Kraft aktiv zu werden und stadtregionale Fragestellungen in der Technologieentwicklung, Wissenschaftsförderung und Politik zu vertreten. Davon ausgehend, dass der räumliche und gesellschaftliche Einsatz der avF nicht ab initio gegeben, sondern erst durch öffentliches und politisches Verhandeln (mit)hervorgebracht wird (Guthrie \& Dutton 1992, s. auch Kap. 4.6), kommt auch der Stadtplanung eine gestalterische Verantwortung zu, da die räumlichen Zielsetzungen während der formativen Phase auf die weitere Entwicklung der Technologie einwirken und diese möglicherweise sogar nachhaltig prägen. Nun stellt sich die dringliche Frage, zu welchem Zeitpunkt konkrete planerische Maßnahmen zu setzen sind und wie der Prozess der dafür nötigen Visions- und Strategiefindung zu gestalten ist.

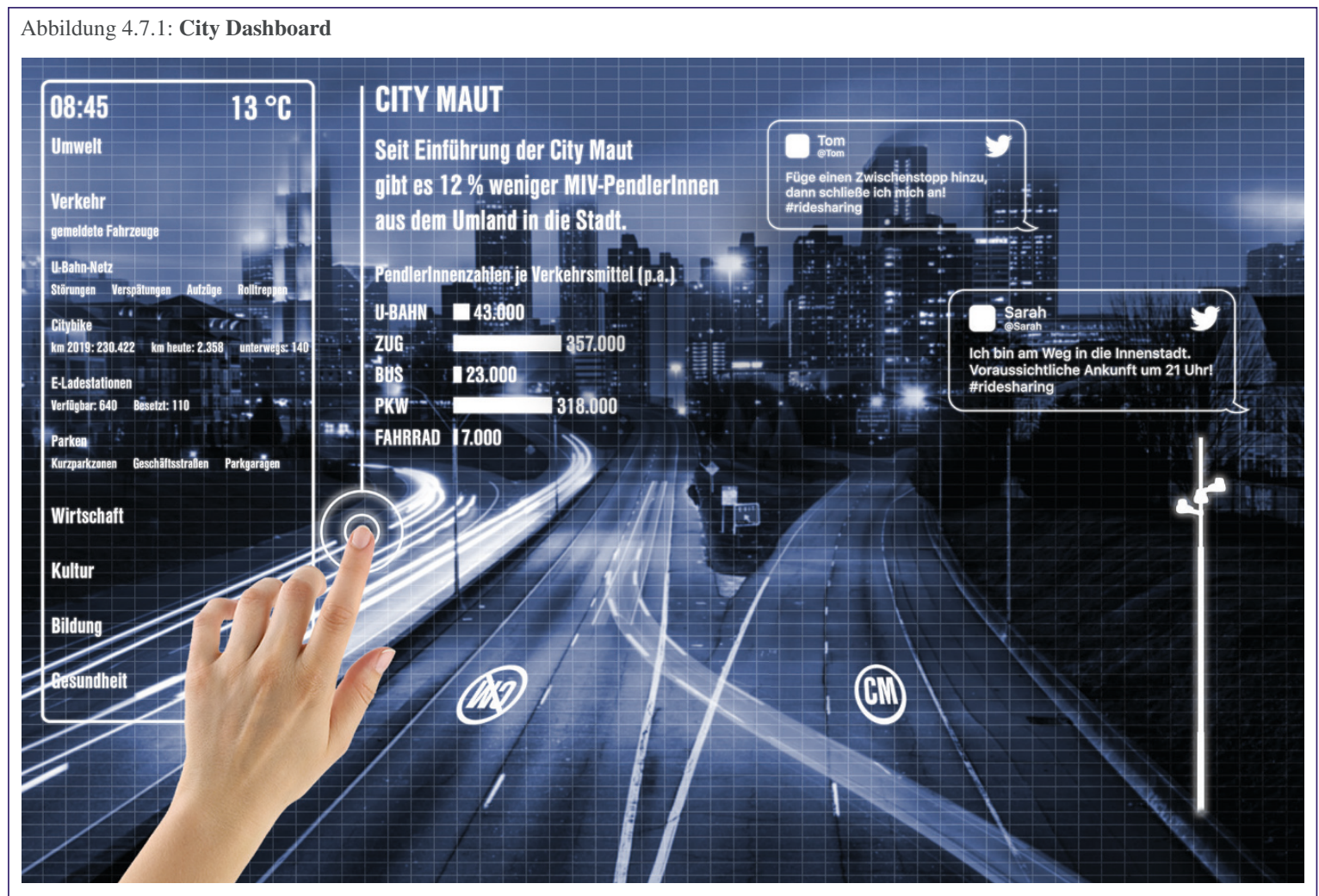

Quelle: AVENUE2 


\subsubsection{TECHNOLOGISCHER WANDEL ALS HERAUS- FORDERUNG FÜR DIE STADTPLANUNG}

Die Einführung der avF gliedert sich in einen Prozess der informationstechnologischen Aufrüstung von zahlreichen europäischen Städten (London, Wien, Amsterdam, Barcelona, Dublin etc.) und internationalen (New York, Singapur, Hongkong, Pune etc.) ein. In den vergangenen beiden Jahrzehnten wurden dazu unter dem Leitmotiv der „Smart City“ Strategiepapiere verfasst, die sich zwar in ihren inhaltlichen Zielsetzungen, Entwicklungsprozessen und Implementierungsstrategien unterscheiden, jedoch weitgehend das Ziel verfolgen, technologische wie auch ökonomische Trends an lokale Entwicklungsbedingungen anzupassen (Townsend \& Lorimer 2015). Angesichts infrastruktureller Kapazitätsgrenzen, fiskalischer Austerität, der Klimakrise und anhaltender Urbanisierungstendenzen wird erhofft, mittels smarter Infrastrukturentwicklung urbane Resilienz und Nachhaltigkeit gewährleisten zu können (White 2016). Mit dem Anspruch, urbane Prozesse in Echtzeit überwachen, verwalten und steuern zu können (Kitchin 2015), hält ein technologieund managementorientierter Planungsansatz Einzug, dem die Überzeugung zugrunde liegt, dass mit ,besseren" Daten und ,besseren" Modellen Unsicherheiten und Risiken erheblich reduziert, wenn nicht gar beseitigt werden können (Hillier 2016, S. 300). Die Vorhersage und Kontrolle von Ereignissen innerhalb von Infrastrukturnetzen oder öffentlichen Räumen (s. Abb. 4.7.1) steht im Zentrum dieses Versprechens (Picon 2015). So stellen die idealisierten Smart City Developments wie Songdo (Südkorea), Masdar (Abu Dhabi) oder jüngst auch Sidewalk Toronto (Kanada) letztlich einen städtebaulichen Ausnahmezustand dar, der ermöglichen soll, sensorische Vernetzung und prognostische Datenanalyse abseits der chaotischen Realität einer Stadt unter Beweis zu stellen, wobei der Zugriff auf Daten zur bestimmenden Kategorie urbaner Transformation erhoben wird (Halpern et al. 2013).

Doch die Einführung von technologischen Neuerungen zum Zweck der Energieversorgung, von Transportsystemen oder Kommunikationsdiensten und die damit einhergehende Anpassung von Städten führt auch im Hinblick auf digitale und automatisierte Prozesse zu unvorhergesehenen Begleiterscheinungen (Townsend 2013, Kitchin $\&$ Dodge 2017). Neben den versprochenen Vorteilen des Komforts, der Sicherheit und des Wirtschaftswachstums ist das Wiederkehren urbaner Problemlagen und Risiken (Aufkommen neuer Ungleichheiten, Sicherheits- und Kriminalitätsrisiken sowie Umweltbelastungen) kaum zu vermeiden (Kitchin \& Dodge 2017). Die sich daraus ableitende Problematik besteht weniger im Einsatz technologischer Neuerungen, sondern vielmehr im Versäumnis, die städtische Vielfalt angemessen zu berücksichtigen und den Nutzen mit den gesellschaftlichen, kulturellen und räumlichen Eigenheiten eines urbanen Kontextes abzustimmen und so auch eine historische Perspektive zu wahren (Hajer 2014, Picon 2015). Angesichts zunehmender Dienstleistungs- und Mobilitätsangebote laufen Städte Gefahr, den Diskurs um die lokalen Bedingungen für eine lebenswerte Stadt im Zeitalter der digitalen Transformation einer übereilten Implementierung von technologischen Lösungsansätzen zum Opfer fallen zu lassen (Hajer 2014, S. 16).

Im konkreten Fall der avM ist zu berücksichtigen, dass den erhofften Optimierungseffekten lokale Pfadabhängigkeiten in Form von Mobilitätskulturen, Siedlungsstrukturen, Wirtschaftsbezügen und bewährten Planungskonzepten entgegenstehen. Die Komplexität der historisch gewachsenen Interdependenzen tendiert dazu, ein ,,automobiles System“ (Urry 2004, S. 27) und so auch eine ,automobile Stadt" zu reproduzieren, dessen Infrastrukturverbesserungen paradoxerweise wiederholt Problemverschiebungen und Rebound-Effekte verursachen (Sonnberger \& Gross 2018, Schneidewind \& Scheck 2013). Um bestehende Mobilitäts- und Konsumpraktiken im Sinne einer nachhaltigen Verkehrswende (s. Kap. 3.1, 3.2) aufzubrechen, ist es vielmehr notwendig, avM im Zusammenspiel mit sozialen Innovationen zu betrachten. Da durch technologische Artefakte immer auch eine gesellschaftliche Ordnung mitentworfen wird, ist soziale Innovation, die als gesellschaftlicher Wandlungsprozess verstanden werden kann, eng an technologische Artefakte gekoppelt (BraunThürmann 2005). Dies bedeutet einerseits, dass der Einsatz von avF nicht als einfache Lösung für zeitgenössische Verkehrs- und Planungsprobleme verstanden werden kann, sondern im Verhältnis zur Stadtstruktur und Gesellschaft kritisch zu beleuchten ist (Guerra 2016). Andererseits sind lokale Planungsstrategien erforderlich, innerhalb derer Unsicherheiten als natürlicher Teil des Prozesses verstanden und unvorhergesehene Entwicklungen als Chance für Neues wahrgenommen werden. Zwar sind Pilotprojekte zur avM auch räumlich und gesellschaftlich möglichst früh zu erproben, doch sollte damit ein öffentlicher Diskurs und Prozess des kollektiven Lernens einhergehen. Um der Frage nachzugehen, wie eine langfristige Vision des Zusammenlebens in Städten mit avF aussehen kann, sind zunächst unterschiedliche Entwicklungsrichtungen auszuloten sowie Erwünschtes und Unerwünschtes zu diskutieren.

\subsubsection{RELEVANZ REFLEXIVER PLANUNGSANSÄTZE VOR DEM HINTERGRUND NEUER MOBILITÄTSTECH- NOLOGIEN}

Angesichts von Unsicherheiten und unvorhersehbaren Entwicklungen, die gemeinhin mit Innovations- und Transformationsprozessen einhergehen, gewinnen anpassungsfähige, explorative und reflexive Ansätze in der Planungstheorie und Praxis an Aufmerksamkeit (Balducci et al. 2011, Roorda et al. 2014, Freudendal \& Kesselring 2016, Bertolini 2017, Hopkins \& Schwanen 2018). So beruht das Verständnis der ,adaptiven“ oder 
auch ,evolutionären“ Planung auf einer inkrementalistischen Erprobung von Neuerungen und losen Regelwerken, um angesichts globaler Herausforderungen urbane Resilienz, d. h. die Wiedererneuerungskraft und die Fähigkeit zur Selbstorganisation im Krisenfall zu gewährleisten (Bertolini 2007, Rauws 2017). Vor dem Hintergrund „robuster Entwicklungsziele“ (Bertolini 2017, S. 147), die trotz Unsicherheiten für unterschiedliche Zukünfte als erstrebenswert bewertet werden können, ist das realweltliche Potenzial technologischer Neuerungen durch explorative Handlungen zunächst zu erproben. Dazu sind Variationen und Auswahlverfahren sowohl in der Konzeptionsphase als auch in späteren Planungs- und Entwicklungsstufen zu berücksichtigen, um aus Erfahrungen lernen und Änderungen vornehmen zu können (Bertolini 2017, S. 156).

Im Diskurs zur „reflexiven Governance“ werden zwei wesentliche Lesarten des Konzepts differenziert (Voß et al. 2006). Zunächst wird Reflexivität als Zustand der Governance in einer modernen Welt verstanden, die fortwährend mit den unbeabsichtigten Folgen früherer Handlungen, ja mit ihren Risiken und Grenzen konfrontiert wird (Voß et al. 2006, Schwarz 2014). ${ }^{1}$ Eine zweite Lesart bezieht sich auf neue Strategien, Prozesse und Institutionen, die durch eine Selbstkonfrontation angeregt werden (Voß \& Kemp 2006). Anstelle von Problemlösungs- und Planungsansätzen der Moderne, die auf wissenschaftlicher Gewissheit und Letztgültigkeit beruhen, tritt in einer ,reflexiven Moderne“ der Diskurs (Schwarz 2014, S. 209). Reflexive Strategien beruhen nach Stirling (2006, S. 260) auf der Pluralität und den Bedingtheiten, denen wissenschaftliche Erkenntnisse ebenso wie technologische Potenziale zugrunde liegen. Die Berücksichtigung langfristiger systemischer Folgen und die Entwicklung alternativer Strategien bedarf daher einer Vielfalt an Perspektiven und einer erweiterten Wissensbasis (Stirling 2006, S. 258). Prinzipien wie die Nichtendgültigkeit, das Experimentieren, die Fehlertoleranz, die Risikointelligenz und das Handeln in der Ungewissheit (Voß et al. 2006, Heidbrink 2007, Schwarz 2014) gewinnen ebenso an Bedeutung wie die Konzeption langfristiger Strategien, die Rahmenbedingungen für kurzfristige, explorative Handlungen schaffen und projektübergreifendes Lernen ermöglichen (Lissandrello \& Grin 2011). Reflexive Strategien sind für die Planung sowohl im Sinne von begleitendem Monitoring als auch der Prozessgestaltung relevant, beispielsweise in Form von offenen Dialogverfahren für die Gestaltung von Zukunftsvisionen oder explorativen urbanen Interventionen. Die für Transformationspfade notwendigen alternativen Praktiken verlangen nicht zuletzt eine Reflexion der jeweiligen strukturellen Rahmenbedingungen (Grin 2006, Lissandrello \& Grin 2011).

Doch nicht nur innerhalb des Planungsdiskurses gewannen in den vergangenen Jahrzehnten urbane Interventionen und Experimentierräume an Aufmerksamkeit (Heyen et al. 2018). Besonders im Kontext der Nach- haltigkeits- und Transformationsforschung wurden realweltliche Erprobungen zum Zweck der angewandten Forschung und Analyse von Wandlungsprozessen verstärkt gefördert (Schneidewind \& Scheck 2013). Städte oder Stadtteile dienen dabei als geographisch, zeitlich und institutionell abgegrenzte Bezugsräume, innerhalb derer durch das Anstoßen und Ermöglichen von sozialen und technologischen Veränderungsprozessen wissenschaftliches und praxisrelevantes Wissen integriert werden kann (Schneidewind \& Scheck 2013, S. 240). Neben den in der deutschsprachigen Transformationsdebatte viel genannten Reallaboren ist eine Reihe von internationalen Forschungsansätzen zu differenzieren, u. a. die (Sustainable oder Urban) Living Labs, die (Urban) Transition Labs oder die (Sustainable) Niche Experiments (Schäpke et al. 2017). ${ }^{2}$ Da in der Forschungslandschaft bislang keine einheitliche methodische Praxis festgelegt wurde, variieren Reallabor-Ansätze im deutschsprachigen Raum bisher hinsichtlich der Ausprägung einzelner Charakteristika wie des Verständnisses transformativer Forschung, der Durchführung von realweltlichen Experimenten, transdisziplinären Kooperationsarten und weitergehender Beiträge zu gesellschaftlichen Wandlungsprozessen durch die Verbreitung und Übertragung von produziertem Wissen (Schäpke et al. 2017).

Werden urbane Reallabore indessen als transdisziplinäre Rahmen verstanden, können diese dazu dienen, unterschiedliche Realexperimente in Bezug zu setzen, einen gemeinsamen Zielhorizont zu eröffnen und einen übergreifenden Erfahrungsaustausch und Reflexionsprozess zu ermöglichen (Beecroft et al. 2018, S. 77). Im Sinne einer ,transformativen Forschung“ sind dabei die Forschungs-, Praxis- und Bildungsziele des Reallabors, der einzelnen Projekte sowie der beteiligten AkteurInnen aufeinander abzustimmen. Auch wenn eine $\mathrm{Zu}-$ sammenarbeit auf gleicher Augenhöhe angestrebt wird, sind Interessens- und Zielkonflikte sowie epistemische Konfrontationen kaum zu vermeiden (Dusseldorp 2017, Singer-Brodowski et al. 2018). Einzelne Realexperimente sind methodisch zwischen der reinen Wissensanwendung und der Wissenserzeugung zu verorten (Schneidewind \& Scheck 2013, S. 241), wobei insbesondere situationsgebundene Settings, ähnlich der „Transition Experiments“, die Kontextualisierung technologischer oder ökologischer Möglichkeiten und die gesellschaftliche Befähigung radikaler Alternativen zum Ziel haben. Ein experimentell-reflexiver Forschungsstil (Beecroft et al. 2018, S. 78) kann durch eine kontinuierliche (Selbst-) Reflexion und Evaluierung dazu beitragen, dass Prozesse an neue Bedingungen oder Unvorhergesehenes angepasst werden und das Reallabor als Ganzes lernfähig wird (Flander et al. 2014).

Angesicht technologischer Neuerungen wie avF stellen Reallabore für die Stadtplanung im Sinne der Transformationsforschung ein relevantes Instrument dar, um mit zivilgesellschaftlicher Beteiligung einen kollektiven Lernprozess zu beginnen. Besonders Themen wie der 
Wandel von Mobilitätsverhalten, die Zugänglichkeit und Barrieren neuer Mobilitätsangebote und städtebauliche Veränderungen eignen sich für derartige Laborsituationen. Die Möglichkeiten und Grenzen, lokal relevantes Wissen zu generieren oder gar einen Wandel anzustoßen, werden von der Integration unterschiedlicher Zieldimensionen sowie der Priorisierung von Forschungsprinzipien bestimmt werden. Für die Stadtentwicklung bleibt es dabei unerlässlich, die gesellschaftliche Legitimität der Zielsetzungen, der Prozessgestaltung und der Ergebnisse fortwährend zu sichern (Schäpke et al. 2017).

\subsubsection{TROTZ UNSICHERHEITEN MIT AUTOMATISIER- TEN UND VERNETZTEN FAHRZEUGEN PLANEN?}

In Hinblick auf die formative Phase der avM und deren urbanen Einsatz können reflexive, explorative und adaptive Prozesse als Teil strategischer Planung von mehrfacher Bedeutung sein. Zunächst, um einer rein technologiegetriebenen Rationalität zu begegnen, die bestrebt ist, mittels schneller, standardisierter Lösungen die Komplexität und Unsicherheiten zu minimieren und die Kontrolle von Risiken zu maximieren. Es gilt vielmehr Planungsansätze zu verfolgen, deren Fokus auf die Bedingungen und nötigen Kapazitäten gerichtet ist, so dass Stadtentwicklung unter unterschiedlichen zukünftigen Umständen erfolgen kann (Rauws 2017). Im Sinne eines ,adaptiven“ oder „,inkrementellen“ Planungsansatzes (Rauws 2017, S. 36) kann mit einem Entwicklungsleitbild ein strategischer Rahmen und ein Orientierungsangebot geschaffen werden, das kontextspezifische Flexibilität bewahrt, ohne im Vorhinein konkrete räumlich-funktionale Entwürfe oder Akteurskoalitionen festzuschreiben. Angesichts der neuen AkteurInnen-Landschaft und des zunehmenden Wissensvorsprungs von IT-Unternehmen ist insbesondere die öffentliche Kompetenzentwicklung und Wissensproduktion notwendig. Neben der Vermittlung von multisektoralen Interessen liegt es an der Stadtplanung, mit der Komplexität urbaner Transformationsprozesse zu planen und Maßnahmen zu verfolgen, die für eine Vielfalt unterschiedlicher Szenarien und urbaner Lebensweisen dienlich sein können (Guerra \& Morris 2018). Es geht dabei weniger um räumliche oder technologische Lösungen als darum, zukunftsoffene Möglichkeitsräume für künftige Weiterentwicklungen einzuplanen, d. h. auch räumliche und gesellschaftliche Anpassungs- und Reaktionsfähigkeit zu stärken.

Zudem sollten eben jene Ansätze, Strukturen und Systeme reflektiert werden, die ein automobiles System hervorgebracht haben und erhalten, um einer Fortschreibung mit avF entgegenzuwirken. Dazu bedarf es proaktiver Planungsansätze, durch die Auseinandersetzungen, Konfrontationen und Perspektivenwechsel zwischen AkteurInnen der Planung, Politik, Wissenschaft, Wirtschaft und Zivilgesellschaft initiiert werden können. In offenen Dialogverfahren und transdisziplinären Projekten, deren Ziel auch die langfristige Transformation sozialer Praktiken darstellt, können AkteurInnen angeregt werden, sich mit bestehenden Herausforderungen und einer notwendigen Anpassung von Handlungsweisen auseinanderzusetzen. Mittels reflexiver Strategien können Problemlagen offengelegt, Erwartungen an die Zukunft konkretisiert und vor dem Hintergrund existierender Routinen strukturelle Zwänge reflektiert werden (Lissandrello \& Grin 2011). Solche Prozesse dienen nicht lediglich dem Austausch zwischen den AkteurInnen, sondern der Integration unterschiedlicher Perspektiven, um eine gemeinsame Sicht auf die Realität zu entwickeln, auch wenn diese vorerst unterschiedliche Problemverständnisse, Ziele und Strategien enthält (Voß et al. 2006). Dabei tritt die Pluralität von Ansichten und die kollektive Produktion von Wissen in den Vordergrund der Zusammenarbeit (Voß \& Kemp 2006). Durch das Aufzeigen lokaler Entwicklungsmöglichkeiten und Synergien zwischen Bestehendem und Neuem gilt es, die Vorstellungskraft von StakeholderInnen neu auszurichten und alternative Zukunftsvisionen für eine Stadtregion oder einen Stadtteil zu ermitteln. Im Idealfall wandeln sich bisherige Verständnisse von urbaner Mobilität, während sich individuelle Ansprüche alternativen Zukunftspfaden annähern (Lissandrello \& Grin 2011, S. 244). So kann Reflexivität in der Planung, die auch das Hinterfragen der Rolle und die zugrunde liegenden Überzeugungen von PlanerInnen selbst miteinschließt (Beecroft et al. 2018, S. 92), durch produktive Konfrontationen einen Veränderungsprozess anstoßen (Lissandrello \& Grin 2011, S. 226). Der Kontext für kritische Auseinandersetzungen mit der Zukunft schafft einen Rahmen für transformative Praktiken, die über kommunikative und strategische Planungsprinzipien hinausgehen.

Letztlich kann die Eignung neuer Mobilitätsangebote für einen bestimmten räumlichen und sozialen Kontext in explorativen Planungsansätzen geprüft oder abgestimmt werden. Bislang erfolgen Testbetriebe hochautomatisierter Fahrzeuge (Level 4) in kontrollierten Orientierungs- und Experimentierrahmen, um übertragbares Wissen zum Zweck der Technologieentwicklung zu ermitteln (s. Kap. 4.1). So sind bisherige Realversuche zur avM zwischen kontrollierten Test-Beds, d. h. Demonstrationsanlagen, und Living Labs, d. h. realweltlichen Erprobungen unter Beteiligung von NutzerInnen, zu verorten (s. Abb. 4.7.2). Aspekte wie der Wandel von Mobilitätsgewohnheiten, stadträumliche Wechselwirkungen und die vielfältigen Nutzungsbedürfnisse von Zielgruppen wie mobilitätseingeschränkten Personen wurden im Zusammenhang mit avM kaum berücksichtigt. Aus Sicht der Stadt und Stadtplanung ist daher weiterführend zu ermitteln, welche Erkenntnisse aus den bisherigen Untersuchungen gewonnen und in welchem Ausmaß künftige Erprobungen methodisch erweitert und mitgestaltet werden können. Exploratives Probehandeln in Form von kurzfristigen urbanen Interventionen oder urbanen Reallaboren kann dazu dienen, transformationsbezogenen Fragen exemplarisch nachzugehen und standortspezifische Erfahrungen zu gewinnen. Die Anforderung sollte allerdings sein, gesellschaftlich legitimierte, ethisch gut begründete und gemeinwohlorien- 


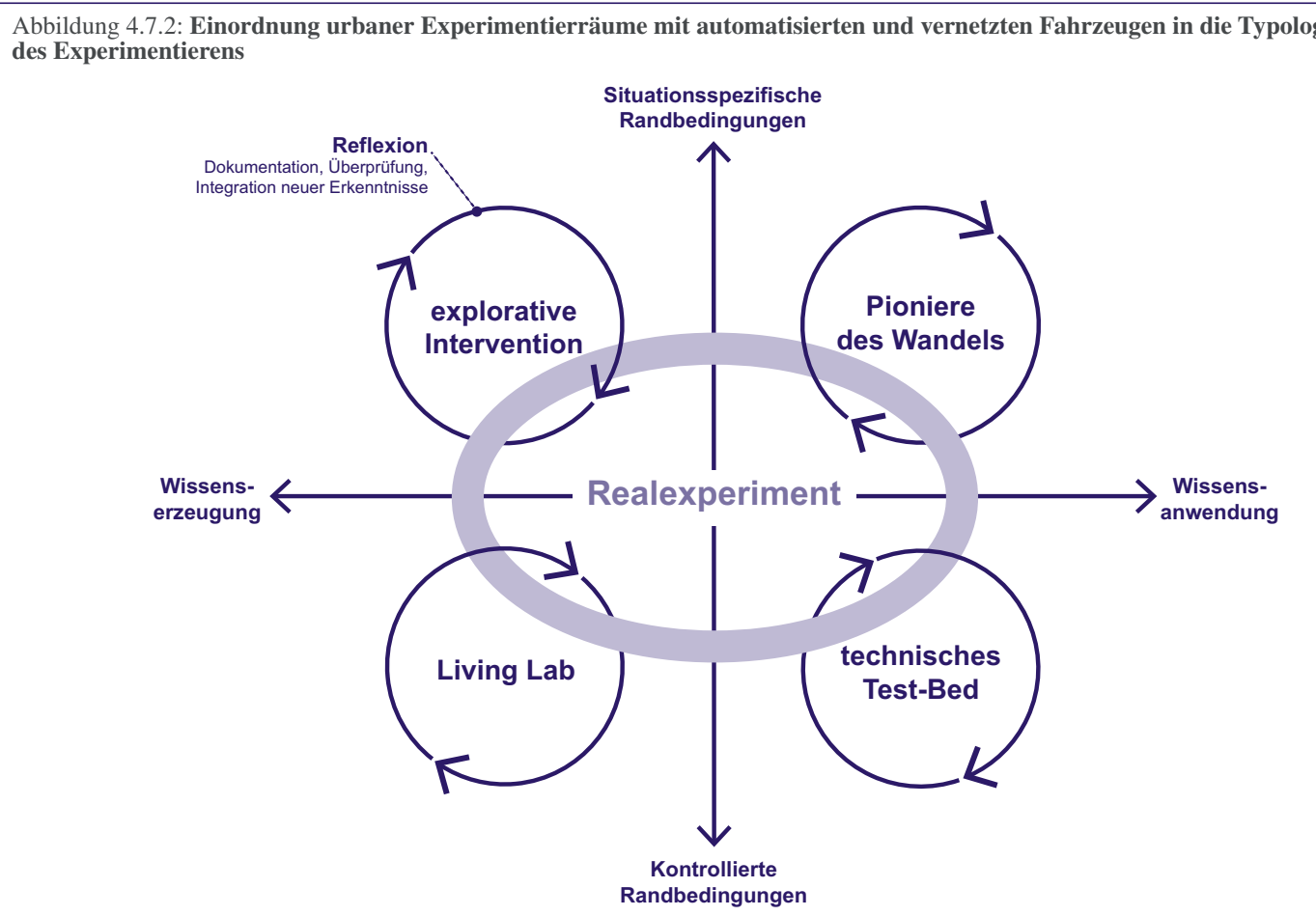

Quelle: AVENUE21 nach Groß et al. (2005, S. 19) und Schneidewind \& Scheck (2013, S. 241)

tierte Ziele zu verfolgen (Defila \& Di Giulio 2018). Aus wissenschaftlicher Perspektive wäre spezifisches System-, Ziel-, Transformations- sowie Prozesswissen lokal zu erheben und durch den Einbezug praxisrelevanter AkteurInnen die gesellschaftliche Relevanz der jeweiligen Forschungsfragen zu überprüfen sowie die Ausrichtung einzelner Realexperimente kollaborativ zu wählen (Beecroft et al. 2018, S. 80). Dazu sind besonders lokale AkteurInnen zu berücksichtigen, die sich mit den Folgen neuer Mobilitätsangebote auf öffentliche Räume, Arbeitsbedingungen, Gemeinschaften, Klima, Bildung, Zugang und Leistbarkeit befassen. Durch das Anstoßen von alternativen Mobilitätspraktiken und unterschiedlichen Nutzungsformen wäre praxisrelevantes Wissen über nichtintendierte Folgen, die Übertragbarkeit von Ergebnissen sowie die Auswirkungen und Erfordernisse von Kooperations- und Ermächtigungsformen zu gewinnen (Beecroft et al. 2018, S. 81). Werden Fragestellungen und Transformationsziele lokal verankert und selbstorganisierte Gruppen ermächtigt, eigene Problemlösungsideen zu entwickeln, können Initiativen entstehen, die kulturellen, sozialen und räumlichen Mehrwert schaffen und im Sinne von „change agents“ oder Pionieren des Wandels einen Kulturwandel anstoßen (WBGU 2011, S. 256). Dazu bedarf es nicht nur der Offenheit gegenüber soziokulturellen Eigenarten und der Zusammenarbeit auf Augenhöhe, sondern auch der Möglichkeit, die Teilhabe lokaler AkteurInnen über den Zeitraum von Projektphasen hinweg zu erhalten. Die systematische Reflexion der $\mathrm{Zu}$ sammenarbeit und der Ergebnisse kann schließlich unterschiedlichen Bildungsprozessen dienen, so beispielsweise der Lern- und Anpassungsfähigkeit innerhalb von Reallaboren oder auch der Ausgestaltung von Transformations- pfaden als gesellschaftliche Lernprozesse im weiteren Sinne (Schneidewind \& Singer-Brodowski 2015). Die kreative Rekonfiguration der Gegenwart und das Experimentieren mit der Änderungsfähigkeit macht reflexive und explorative Verfahren zu wertvollen Nischen, in denen die Grenzen des Möglichen neu ausgelotet werden und urbane Diversität als Qualität erhalten bleibt (Abbott 2012).

1 Hierbei wird auf die ,reflexive Moderne“ nach Beck (1986) und weitere (Beck et al. 2003) verwiesen, womit die grundlegende Transformation einer modernen Gesellschaft und ihrer politischen Systeme gemeint ist. Im Gegensatz zu den Auffassungen einer Postmoderne gehen die AutorInnen mit dem Konzept der reflexiven Moderne von einer Restrukturierung und Rekonzeptualisierung gesellschaftlicher Verhältnisse und geschichtlicher Brüche aus. Nach Latour (2003) ist mit Reflexivität nicht die Zunahme von Beherrschbarkeit und Bewusstsein gemeint, sondern die Erkenntnis, dass eine vollkommene Beherrschbarkeit faktisch unmöglich ist.

2 Nach Schäpke et al. (2017, S. 49) sind die drei Ansätze im Hinblick auf methodische Zugänge, Fokusse und zugrunde liegende Theorien zu unterscheiden: 1.) „Living Labs“ zielen auf marktgängige, standardisierte Produkte und Dienstleistungen sowie generalisierbare Erkenntnisse ab - daher beschränken sie die Partizipation und streben kontrollierte Experimente und Settings an. 2.) „Transition Experiments“ wiederum zielen auf evolutionäre Kräfte, Befähigung von VorreiterInnen und Stärkung von Alternativen - ebenso wie Orchestrierung von Experimenten und konkretem, realweltlichem Wandel. Dementsprechend fokussiert der Ansatz auf starke Formen der Beteiligung und befähigende Mechanismen der Ausbreitung von Alternativen durch Lernen. 3.) „Niche Experiments" tragen durch den Verzicht auf die eigene Durchführung von Experimenten zur Generierung von reflexivem Wissen bei. Dieses ist je nach untersuchten Projekten und Prozessen stark kontextualisiert oder stärker verallgemeinerbar. 
Open Access Dieses Kapitel wird unter der Creative Commons Namensnennung 4.0 International Lizenz (http://creativecommons.org/licenses/by/4.0/deed.de) veröffentlicht, welche die Nutzung, Vervielfältigung, Bearbeitung, Verbreitung und Wiedergabe in jeglichem Medium und Format erlaubt, sofern Sie den/die ursprünglichen Autor(en) und die Quelle ordnungsgemäß nennen, einen Link zur Creative Commons Lizenz beifügen und angeben, ob Änderungen vorgenommen wurden.

Die in diesem Kapitel enthaltenen Bilder und sonstiges Drittmaterial unterliegen ebenfalls der genannten Creative Commons Lizenz, sofern sich aus der Abbildungslegende nichts anderes ergibt. Sofern das betreffende Material nicht unter der genannten Creative Commons Lizenz steht und die betreffende Handlung nicht nach gesetzlichen Vorschriften erlaubt ist, ist für die oben aufgeführten Weiterverwendungen des Materials die Einwilligung des jeweiligen Rechteinhabers einzuholen.

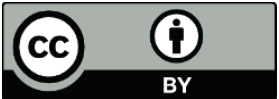

